\title{
POLARIZATION AND CHARGE LIMIT STUDIES OF STRAINED GaAs PHOTOCATHODES*
}

Paḅlo J. Sáez

Stanford Linear Accelerator Center Stanford University

Stanford, CA 94309

RECEIVEO
JUW 17 T997

OSTI

SLAC-Report-501

March 1997

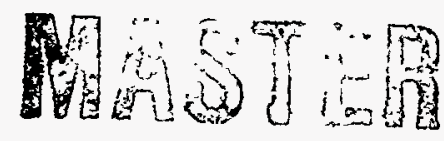

Prepared for the Department of Energy

under contract number DE-AC03-76SF00515

Printed in the United States of America. Available from the National Technical Information Service, U.S. Department of Commerce, 5285 Port Royal Road, Springfield, VA 22161.

"Ph.D. thesis, Stanford University

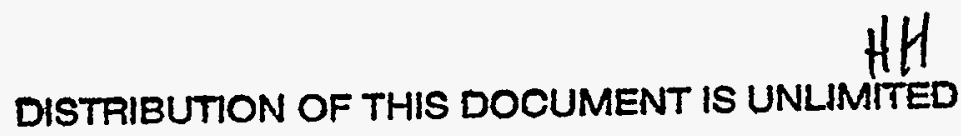




\section{DISCLAIMER}

This report was prepared as an account of work sponsored by an agency of the United States Government. Neither the United States Government nor any agency thereof, nor any of their employees, make any warranty, express or implied, or assumes any legal liability or responsibility for the accuracy, completeness, or usefulness of any information, apparatus, product, or process disclosed, or represents that its use would not infringe privately owned rights. Reference herein to any specific commercial product, process, or service by trade name, trademark, manufacturer, or otherwise does not necessarily constitute or imply its endorsement, recommendation, or favoring by the United States Government or any agency thereof. The views and opinions of authors expressed herein do not necessarily state or reflect those of the United States Government or any agency thereof. 


\section{DISCLAMIER}

Portions of this document may be illegible in electronic image products. Images are produced from the best available original document. 


\section{Abstract}

This thesis presents studies on the polarization and charge limit behavior of electron beams produced by strained GaAs photocathodes. These photocathodes are the source of highintensity, high-polarization electron beams used for a variety of high-energy physics experiments at the Stanford Linear Accelerator Center. Recent developments on p-type, biaxially-strained GaAs photocathodes have produced longitudinal polarization in excess of $80 \%$ while yielding beam intensities of $\sim 2.5 \mathrm{~A} / \mathrm{cm}^{2}$ at an operating voltage of $120 \mathrm{kV}$. The SLAC Gun Test Laboratory, which has a replica of the SLAC injector, was upgraded with a Mott polarimeter to study the polarization properties of photocathodes operating in a highvoltage $\mathrm{DC}$ gun. Both the maximum beam polarization and the maximum charge obtainable from these photocathodes have shown a strong dependence on the wavelength of illumination, on the doping concentration, and on the negative electron affinity levels. The experiments performed for this thesis included studying the effects of temperature, cesiation, quantum efficiency, and laser intensity on the polarization of high-intensity beams. It was found that, although low temperatures have been shown to reduce the spin relaxation rate in bulk semiconductors, they don't have a large impact on the polarization of thin photocathodes. It seems that the short active region in thin photocathodes does not allow spin relaxation mechanisms enough time to cause depolarization. Previous observations that lower $\mathrm{QE}$ areas on the photocathode yield higher polarization beams were confirmed. In addition, high-intensity, small-area laser pulses were shown to produce lower polarization beams. Based on these results, together with some findings in the existing literature, a new proposal for a high-intensity, high-polarization photocathode is given. It is hoped that the results of this thesis will promote further investigation on the properties of GaAs photocathodes and that it will promote the development of highintensity, high-polarization photocathodes. 


\section{Acknowledgments}

Many people contributed to work included in this thesis and without their help this project would have been much more difficult or nearly impossible. The physicists who taught me the most about the SLAC injector, Mott scattering and photocathode physics included Roger Miller, Charles Prescott, Jim Clendenin, John Sheppard, and William Spicer. They are not only great mentors but they are also influential figures in the accelerator, highenergy, and semiconductor physics communities. Other physicists and engineers that made immense contributions to the SLAC Polarized Electron Source project during my time at SLAC include: Ray Alley, Hideki Aoyagi, Joseph Frisch, Christina Garden, Edward Garwin, Earl Hoyt, Robert Kirby, Lowell Klaisner, Eugene Kraft, Robin Mair, Takashi Maruyama, Greg Mulhollan, Michelle Talbert, Huan Tang, James Turner, Dave Schultz, and Klaus Witte. Some of the talented engineers and technicians that contributed to the outstanding performance of the electron source through the years include: David Brown, Mike Browne, Gerard Collet, Stephan Davies, Jeffrey de Lamare, Fred Dusel, Kay Fox, Thomas Galetto (the ever cheerful office and lunch mate), Rupert Gutierrez, Mark Hoyt, Bill Kaminskas, Eric Lundahl, Doug McCormick, Ossie Millican, Kathy Ratcliff, Mike Robertson, Bill Roster, Nancy Spencer, and the late George Tunis. I am also grateful to the people who helped me with the administrative aspect of life at SLAC and with the manuscript and illustrations: Terry Anderson, Kathy Chan, Maura Chatwell, and Crystal Tilghman. I am also grateful to Matt Kessler for editing the final manuscript of this thesis.

Higher education, as important as it is, can also bring difficult separation to a family in trying times. For this reason I feel most indebted to my mother, Ana Maria, my father, Ruben, my sister, Mónica, my brother, Ruben, my aunt, Imelda, and my uncle, Gabriel, all of whom were extremely supportive of my enterprise. Finally, I am infinitely grateful to my girlfriend Karen Ward and her family for being very supportive and patient during this long and difficult pursuit.

Again, thanks to all who made this work possible.

This work was supported by the Department of Energy contract DE-AC03-76SF00515. 


\section{Contents}

1 Introduction 1

2 Physics of GaAs Photocathodes - 4

2.1 GaAs Properties ........................ 5

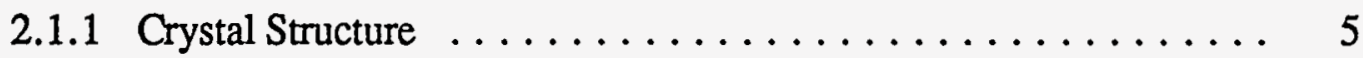

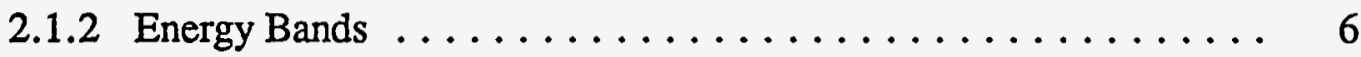

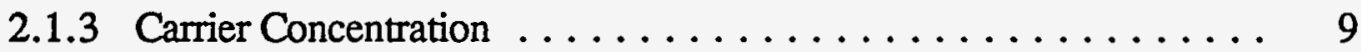

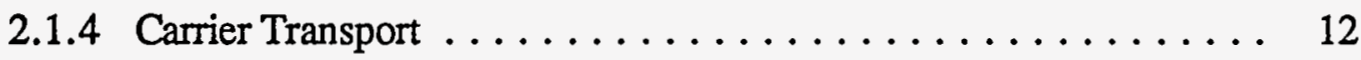

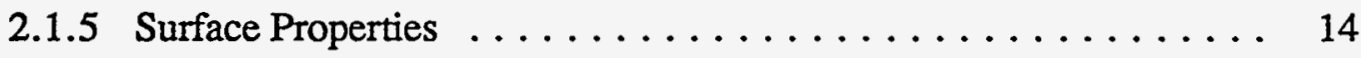

2.2 Photoemission........................... 20

2.2.1 Three-Step Model . . . . . . . . . . . . . . . . . . 21

2.2.2 NEA Semiconductors. . . . . . . . . . . . . . . 24

2.3 Polarized Electrons (include e-polarization definition)........... 26

2.3.1 Strained GaAs. ........................ 27

2.3.2 Depolarization Mechanisms................ 30

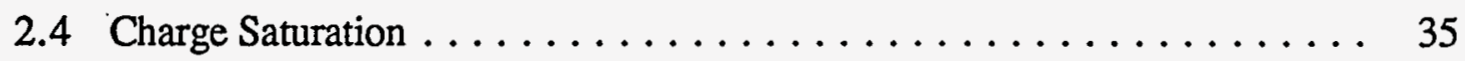

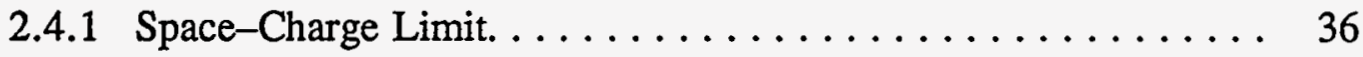

2.4.2 Charge Limit Effect.................. 37

3 The Gun Test Laboratory $\quad 38$

3.1 Gun and Loadlock. . . . . . . . . . . . . . 41

3.2 Injector Beamline. ..................... 45

3.3 Mott Polarimeter Beamline $\ldots \ldots \ldots \ldots \ldots \ldots \ldots \ldots \ldots \ldots .46$

3.3.1 Fast Faraday Cup. .................. 46

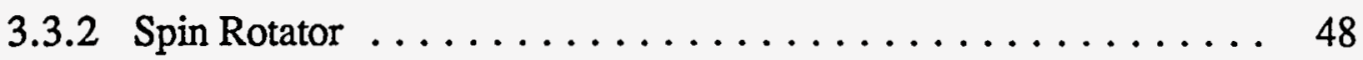

3.3.3 Mott Polarimeter $\ldots \ldots \ldots \ldots \ldots \ldots \ldots \ldots \ldots \ldots \ldots \ldots$ 
4 Experimental , Results $\quad \mathbf{6 0}$

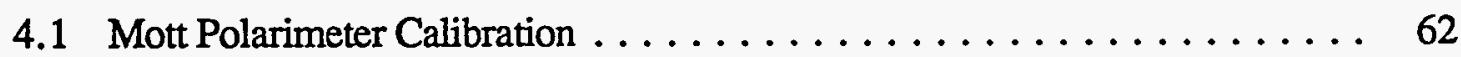

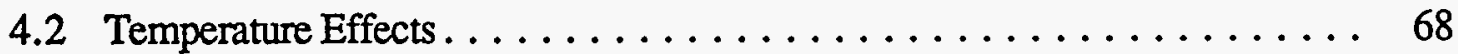

4.3 Polarization Sensitivity to Quantum Efficiency ............. 75

4.4 Charge Limit Effects . . . . . . . . . . . . . . . . . . 79

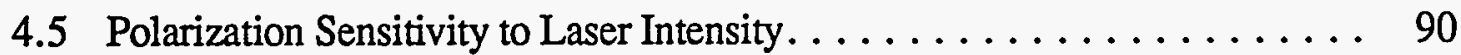

5 Conclusion $\quad 98$

A Electromagnetic Transitions in GaAs 103

B Stress Perturbation of the GaAs Crystal Lattice 106

C Motion of the Electron Spin 113 


\section{List of Tables}

2.1 Some reference values of the $\mathrm{GaAs}$ crystal . . . . . . . . . . . . . 5

2.2 Temperature dependence of the intrinsic GaAs bandgap energy . . . . . . . 8

3.1 Angle of trajectory deflection and electrode voltage required to produce beams

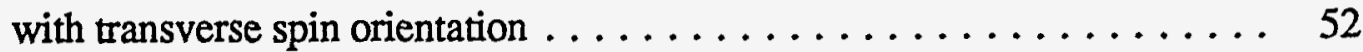

4.1 Polarization of the standard GaAs photocathode measured at the various academic institutions ....................... 64 


\section{List of Figures}

2.1 Symmetry planes of the GaAs crystal lattice . . . . . . . . . . 6

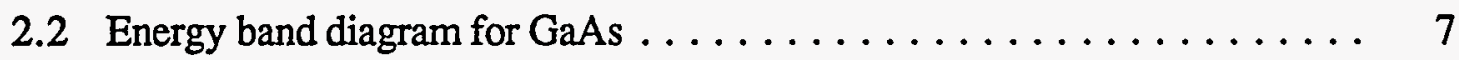

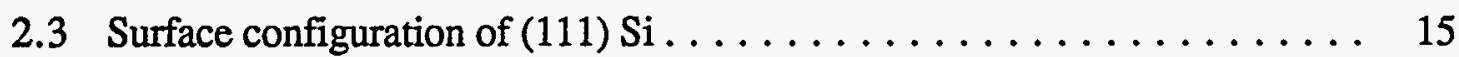

2.4 Depletion region in semiconductors $\ldots \ldots \ldots \ldots \ldots \ldots \ldots \ldots \ldots, 17$

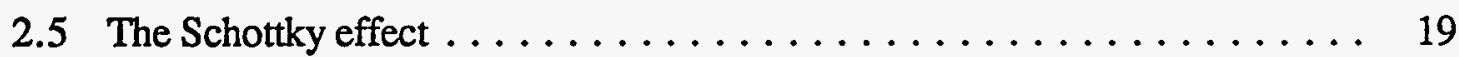

2.6 Spicer's Three-Step Photoemission Process $\ldots \ldots \ldots \ldots \ldots \ldots \ldots .21$

2.7 Optical absorption coefficient for some NEA semiconductors . . . . . . 22

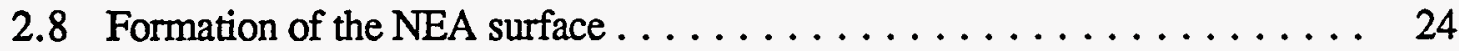

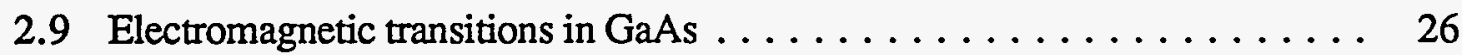

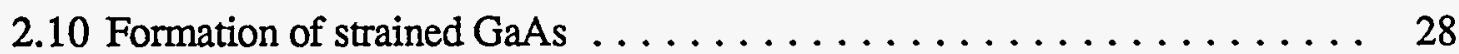

2.11 Spin relaxation rate as a function of temperature for high dopant concentration $\mathrm{GaSb}$ and $\mathrm{GaAs} \ldots \ldots \ldots \ldots \ldots \ldots \ldots \ldots \ldots$

2.12 Spin relaxation rate as a function of temperature in low doped GaAs . . . 34

2.13 Planar electrodes separated by a distance $d$ and held at potential $\phi=-V_{0}$

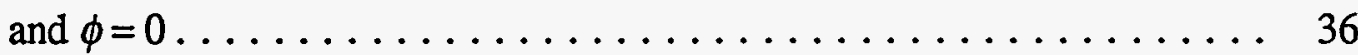

3.1 Layout of the SLAC Gun Test Laboratory . . . . . . . . . . 39

3.2 Schematic of the SLAC polarized-electron gun . . . . . . . . . 41

3.3 Schematic of the Pierce-type electrodes for the SLAC polarized-electron gun 42

3.4 Schematic of the SLAC loadlock system . . . . . . . . . . . 44

3.5 Transmission of a diffracted beam pattern in the GTL beamline . . . . . . 45

3.6 Schematic of the fast Faraday cup $\ldots \ldots \ldots \ldots \ldots \ldots \ldots \ldots \ldots$

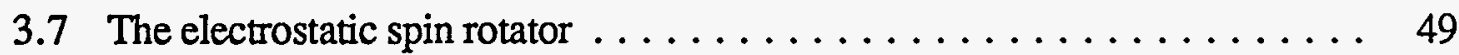

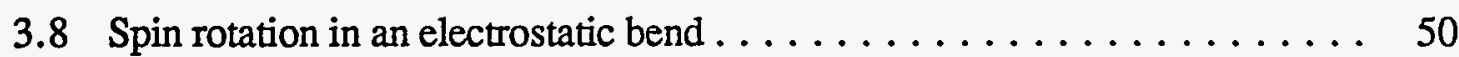

3.9 Alignment of the beam spots to the optic axis of the spin rotator . . . . . 52

3.10 Measurement of an electron beam polarization with Mott scattering ..... 55

3.11 Target holder in the GTL Mott polarimeter . . . . . . . . . . 57

3.12 Profile of the standard GaAs photocathode $\ldots \ldots \ldots \ldots \ldots \ldots \ldots . \ldots \ldots$ 
4.1 Polarization and $\mathrm{QE}$ as a function of wavelength of illumination for the standard $\mathrm{GaAs}$ photocathode . . . . . . . . . . . . . . . 63

4.2 Electron beam impinging on the alignment target inside the Mott chamber . . 65

4.3 Calibration points taken from the standard $\mathrm{GaAs}$ photocathode . . . . . . 67

4.4 Cross-section of the polarized-electron gun electrode illustrating the cooling

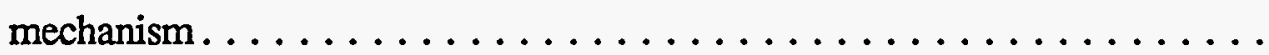

4.5 Temperature at the end of emitter tube versus the average temperature of the cooling nitrogen gas $\ldots \ldots \ldots \ldots \ldots \ldots \ldots \ldots \ldots \ldots \ldots \ldots \ldots \ldots$

4.6 $\mathrm{QE}$ dependence on temperature for bulk GaAs ............. 71

4.7 $\mathrm{QE}$ dependence on temperature for thin (strained) GaAs . . . . . . . 72

4.8 Polarization dependence on temperature for the $100 \mathrm{~nm}$ standard $\mathrm{GaAs}$

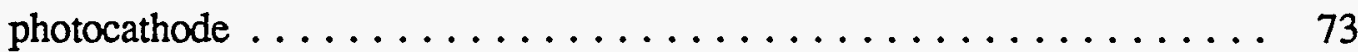

4.9 Polarization dependence on temperature for the $100 \mathrm{~nm}$ strained GaAs photocathode ........................... 74

4.10 Polarization and $\mathrm{QE}$ of a strained GaAs photocathode before and after

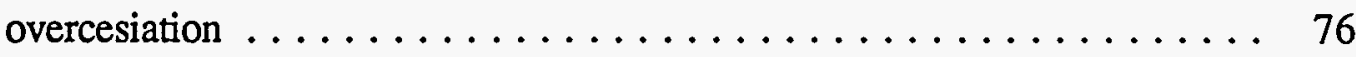

4.11 Depolarization at the surface / band-bending region. . . . . . . . 77

4.12 Effective energy band structure in strained GaAs photocathodes $\ldots \ldots \ldots 78$

4.13 Photoemission of electrons from a broader conduction band . . . . . . . 79

4.14 Maximum charge per bunch as a function of $\mathrm{QE}$ for a $14 \mathrm{~mm}$ diameter bulk GaAs photocathode .................... 80

4.15 Temporal profile of a severely charge-limited electron bunch $\ldots \ldots \ldots 81$

4.16 Effect of bunch \#1 on the maximum charge in bunch \#2 (bunch-bunch effect) 82

4.17 Energy band diagram illustrating a semiconductor undergoing linear response photoemission $\ldots \ldots \ldots \ldots \ldots \ldots \ldots \ldots \ldots \ldots \ldots \ldots . \ldots \ldots$

4.18 Energy band diagram illustrating a semiconductor with diminishing photoemission yield...................... 84

4.19 Saturation plots of $300 \mathrm{~nm}$ strained GaAs for various $\mathrm{QE}$ levels $\ldots \ldots \ldots \quad 85$

4.20 Saturation plots of $300 \mathrm{~nm}$ strained $\mathrm{GaAs}$ for various $\mathrm{QE}$ levels $\ldots \ldots \quad 86$

4.21 Effect of the $\mathrm{CL}$ on laser pulses with different time profiles . . . . . . . 87

4.22 Polarization dependence on laser intensity for different laser spot sizes . . . 91

4.23 Polarization dependence on laser intensity for a $200 \mathrm{~nm}$ strained GaAs

viii 
photocathode $\ldots \ldots \ldots \ldots \ldots \ldots \ldots \ldots \ldots \ldots \ldots . . \ldots 2$

4.24 Electron bunch profile and polarization for a $1 \mathrm{~mm}$ laser spot with increasing

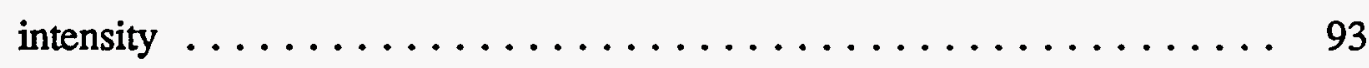

4.25 Polarization of a charge-limited electron bunch scanned across the FFC

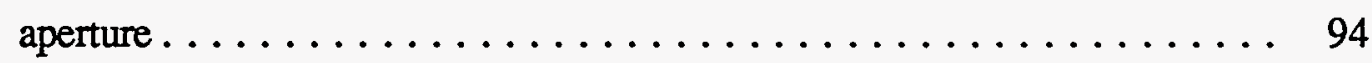

4.26 Vacuum level in a semiconductor experiencing CL . . . . . . . 96

5.1 Band-bending region for various $\mathrm{GaAs}$ surface orientations . . . . . . 100

5.2 $\mathrm{QE}$ dependence on surface orientation for $\mathrm{GaAs}$ photocathodes . . . . . . 101

A.1 Electromagnetic transitions at the $\Gamma$ point of $\mathrm{GaAs}$ for $T=0^{\circ} \mathrm{K} \ldots \ldots \ldots 103$

B.1 Formation of shear stain in the GaAs crystal lattice $\ldots \ldots \ldots \ldots \ldots 110$ 


\section{Chapter 1}

\section{Introduction}

Experiments in high-energy physics are performed at the Stanford Linear Accelerator Center (SLAC) to study the fundamental properties of matter. These experiments include the study of the left/right asymmetry in the decay of $Z_{0}$ bosons and the study of the spin structure of the proton. The luminosity necessary for the production of relevant asymmetry events at the interaction point is proportional to $I P^{2}$, where $I$ is the electron.beam intensity and $P$ its average longitudinal polarization. This beam polarization is defined as

$$
P=\frac{N_{+}-N_{-}}{N_{+}+N_{-}}
$$

where $N_{+}$and $N_{-}$are the number of electrons that yield a spin value of $+\hbar / 2$ and $-\hbar / 2$, respectively, when measured with regard to the beam axis.[1] In order to obtain the high luminosity required by these experiments, SLAC requires a reliable and efficient source of intense and highly polarized electrons.

The Gun Test Laboratory (GTL) was built to test and study the production of intense polarized-electron beams. The development of a high performance injector is crucial for the operation of the SLAC Linear Collider (SLC) as well as for the next generation of (high energy) linear colliders (NLC). The GTL has a replica of the first two meters of the SLAC injector. The source of electrons is a high-voltage (HV) photocathode gun. In addition, the GTL has been upgraded with a Mott polarimeter to study the beam polarization from various photocathodes. The charge limit, or saturation under intense laser illumination, and the spin relaxation of electrons during the photoemission process, are two problems which make the production of high-intensity, high-polarization electron beams from semiconductor photocathodes difficult. 
Semiconductors of the III-V family, such as GaAs, are good candidates for the production of polarized electrons due to their well defined energy band structure and to their ability to achieve high photoemission yields.[2] The photoemission efficiency of a photocathode, also known as the quantum efficiency (QE), is defined as the number of emitted electrons per incident photon. The $\mathrm{QE}$ depends strongly on the wavelength of illumination and on the negative-electron-affinity (NEA) levels at the surface of the photocathode (see section 2.2). The highly polarized electron beams are produced by illuminating the photocathodes with circularly-polarized light of energy equal to the semiconductor bandgap. Unfortunately, both the $\mathrm{QE}$ and the beam polarization are very sensitive to the wavelength of illumination near the bandgap, but in opposite respects; shorter wavelengths improve the $\mathrm{QE}$ while lowering the polarization and vice versa for longer wavelengths.

The photocathodes in the SLC guns have achieved space-charge-limited beams of 6.7 Amps $/ \mathrm{cm}^{2}$ at a HV of $120 \mathrm{kV}$. However, when the wavelength of illumination is near the semiconductor bandgap, the maximum beam intensity is below the expected spacecharge limit and depends strongly on the $\mathrm{QE}$ of the photocathode (see section 2.4).[3] This charge saturation, better known as charge limit (CL), was discovered at SLAC. The CL is of great concern to the accelerator group because it sets a limit on the charge that can be obtained at a given operating HV. Understanding the causes of the $\mathrm{CL}$ are very important to further the development of these photocathodes. The CL will be discussed in detail in Chapters 2, 4 and 5.

The high-polarization beams are created by exciting into photoemission electrons of a particular spin state. The spin selection is achieved by breaking the heavy hole (HH)light hole $(\mathrm{LH})$ degeneracy at the $\Gamma$ point (momentum vector $k=0$ ) of the $\mathrm{GaAs}$ valence band.[4] The degeneracy is broken by eliminating the crystal lattice symmetry through compressive biaxial strain: a thin layer of GaAs is grown on a smaller lattice constant $\mathrm{GaAs}_{1-\mathrm{x}} \mathrm{P}_{\mathrm{x}}$. The resulting valence band splitting is on the order of $\sim 50 \mathrm{meV}$. By illuminating the photocathode with circularly polarized light of one helecity, only the electromagnetic transition which conserves angular momentum is allowed (see section 2.3). Beam polarizations in excess of $80 \%$ have been achieved with these strained photocathodes. 
Further improvement of the beam polarization is of great interest, not only to the accelerator community, but to the semiconductor physics community as well. Many physicists believe that the polarization of the SLAC photocathodes would be closer to $100 \%$ if it were not for the problems of strain relaxation or growth defects in the GaAs. However, other mechanisms that can cause spin relaxation have been suggested and might be responsible for part of the imperfect polarization.[5] For-instance, the time spent by the photoexcited electrons in the conduction band is an important factor that can cause spin relaxation. In addition, the spin relaxation mechanisms in the conduction band have a strong temperature dependence. Chapter 4 discusses various experiments performed in the GTL to help determine how externally controlled parameters (such as temperature, QE, cesium layer, laser intensity, etc.) affect the beam polarization of strained photocathodes.

The main purpose of this dissertation is to learn more about the properties of these fascinating semiconductors. A better understanding of these materials will help in the development of photocathodes that yield higher polarization and higher beam intensities. Chapter 2 starts the discussion of the inner workings of strained photocathodes with a review of those GaAs properties relevant to photoemission and electron polarization. Also included in this chapter are discussions on charge saturation and on the various spin relaxation mechanisms. Chapter 3 includes a description of the GTL and its instrumentation. Special emphasis is placed on the design of the Mott beamline which was built in great part for this thesis. Chapter 4 describes the tests performed on these photocathodes and discusses the consequences that the new observations have on the theory of operation of these semiconductors. At the end of the dissertation, Chapter 5 summarizes the results and suggests new experiments that will help in the development of high polarization and high intensity photocathodes. 


\section{Chapter 2}

\section{Physics of GaAs Photocathodes}

Photosensitive devices play an important role in the basic sciences, communications, medicine, engineering and defense. Of particular interest to SLAC is that some of these materials are extremely efficient electron emitters and hence they can be used as electron sources in the accelerator. Semiconductors have the additional advantage over metals in that their ordered crystal arrangement can be engineered to accommodate specific needs. For instance, they can be made sensitive to only a narrow range of wavelength of illumination. At SLAC and at other accelerator facilities these crystals are designed to emit electrons with a specific polarization state.

Direct-bandgap semiconductors of the III-V family are especially good electron emitters due to their high photon absorption rate, long diffusion length, and their ability to achieve negative electron affinity (NEA).[2] In p-type materials that have been activated to NEA levels, electrons photoexcited to the conduction band can escape easily because there is virtually no potential barrier at the surface (the vacuum level lies below the conduction band minimum). In particular, GaAs is the most widely used photoemitter because it has a large direct bandgap (which leads to a high photon absorption rate) and is able achieve large NEA levels. Theory has shown that other III-V compounds made of three or more elements can achieve even larger direct-bandgaps than GaAs. However, difficulty in their manufacturing has prevented accomplishing the theoretical expectations.

Recently, the most important photocathode application for linear colliders has been the production of polarized-electron beams. Beam polarizations approaching $50 \%$ can be produced by illuminating a GaAs photocathodes with circularly polarized light of energy close to the semiconductor bandgap. Furthermore, beam polarizations above $80 \%$ can be obtained by breaking the valence band degeneracy at $k=0$ through biaxial compressive stress of the GaAs crystal lattice. 
This chapter will discuss the physics involved in the production of polarizedelectron beams from NEA semiconductors. In particular, it will focus on the problems in achieving space-charge-limited beams and on the various spin-relaxation mechanisms that electrons experience during the photoemission process.

\subsection{GaAs Properties}

The main advantage of GaAs over other III-V semiconductors is that it possesses the largest direct bandgap. This property reduces the number of ionized impurities in the conduction band and hence virtually eliminates any dark current.[6] The large bandgap also creates a larger NEA level because the conduction band minimum is farther removed from the vacuum level of the cesium-oxide layer.[7] This section will discuss some of the most relevant physical properties of GaAs semiconductors including crystal structure, charge transport, surface states, and bandgap dependence on temperature.

\subsubsection{Crystal Structure}

The crystal lattice structure of GaAs has a zincblende symmetry in which each atom is surrounded by four equidistant neighbors which lie at the corners of a tetrahedron.[8] The bond between two nearest neighbors is composed of two electrons with opposite spins. The zincblende lattice is considered an interpenetrating face-centered cube (i.e. a facecentered cube with atoms inside). Table 2.1 includes some reference values for the GaAs

\begin{tabular}{|l|l|}
\hline Gallium & $Z=31$, Family: IIIA \\
\hline Arsenic & $Z=33$, Family: VA \\
\hline Lattice constant & $a=5.65 \AA$ \\
\hline Nearest neighbor distance & $r_{0}=\sqrt{3} a / 4=2.45 \AA$ \\
\hline Angle of separation between bonds & $109.47^{\circ}$ \\
\hline Crystal density & $5.32 \mathrm{~g} / \mathrm{cm}^{3}$ \\
\hline
\end{tabular}

Table 2.1: Some reference values of the GaAs crystal. 

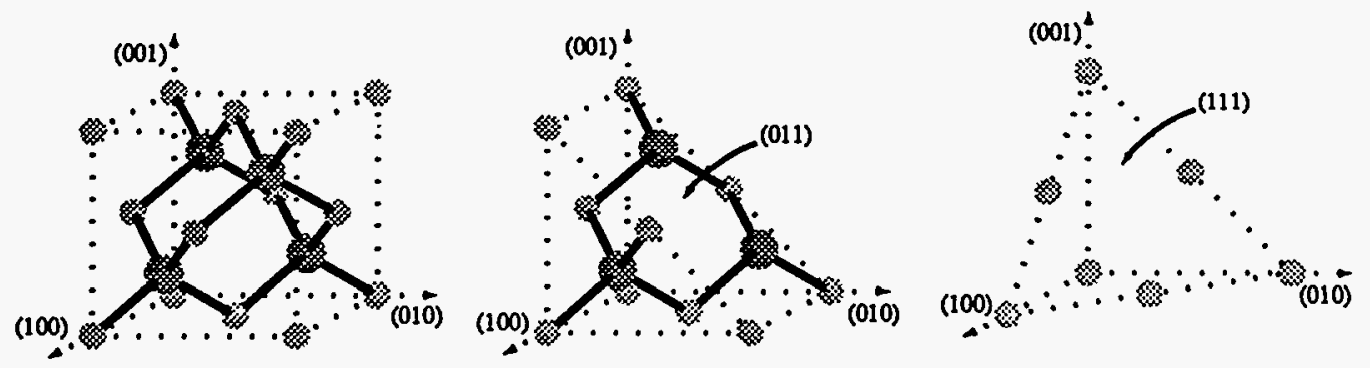

Figure 2.1: Symmetry planes of the GaAs crystal lattice.

crystal.[9] The zincblende lattice structure with the three most important symmetry planes in the GaAs crystal is shown in Fig. 2.1. The crystal planes are defined by the Miller indices $(h k l)$. These indices are determined from the intercepts of the plane, expressed in terms of the lattice constants, with the three basic axes. The Miller indices are the reciprocal of these intercepts reduced to the smallest three integers having the same ratio. The surface plane used in most photocathodes at SLAC is the (100). However, the (100) surface plane can reconstruct to a (110) plane during the surface cleaning process if the semiconductor is heated to a very high temperature.[10] The GaAs crystal cleaves most easily on the (110) plane. This plane also contains both kinds of atoms and it has the closest spacing between atoms.

\subsubsection{Energy Bands}

The energy-momentum relationship of an electron in a periodic crystal can be obtained by solving the Schrödinger equation with a potential that represents the periodicity of the lattice. The solution for most semiconductors includes a set of upper energy bands, known as conduction bands, and a set of lower energy bands, known as valence bands. The region between these two sets of bands is forbidden and no states are allowed to exist. The separation between the bottom of the conduction band and the top of the valence band is called the bandgap energy (or just bandgap). The energy band diagram for $\mathrm{GaAs}$ is shown in Fig. 2.2. The upper valence bands have angular momentum symmetry $L=1$ (i.e. it is three-fold degenerate when spin is not taken into account). With spin included, the bands split due to the symmetry breaking of the spin-orbit interaction. The top two valence 


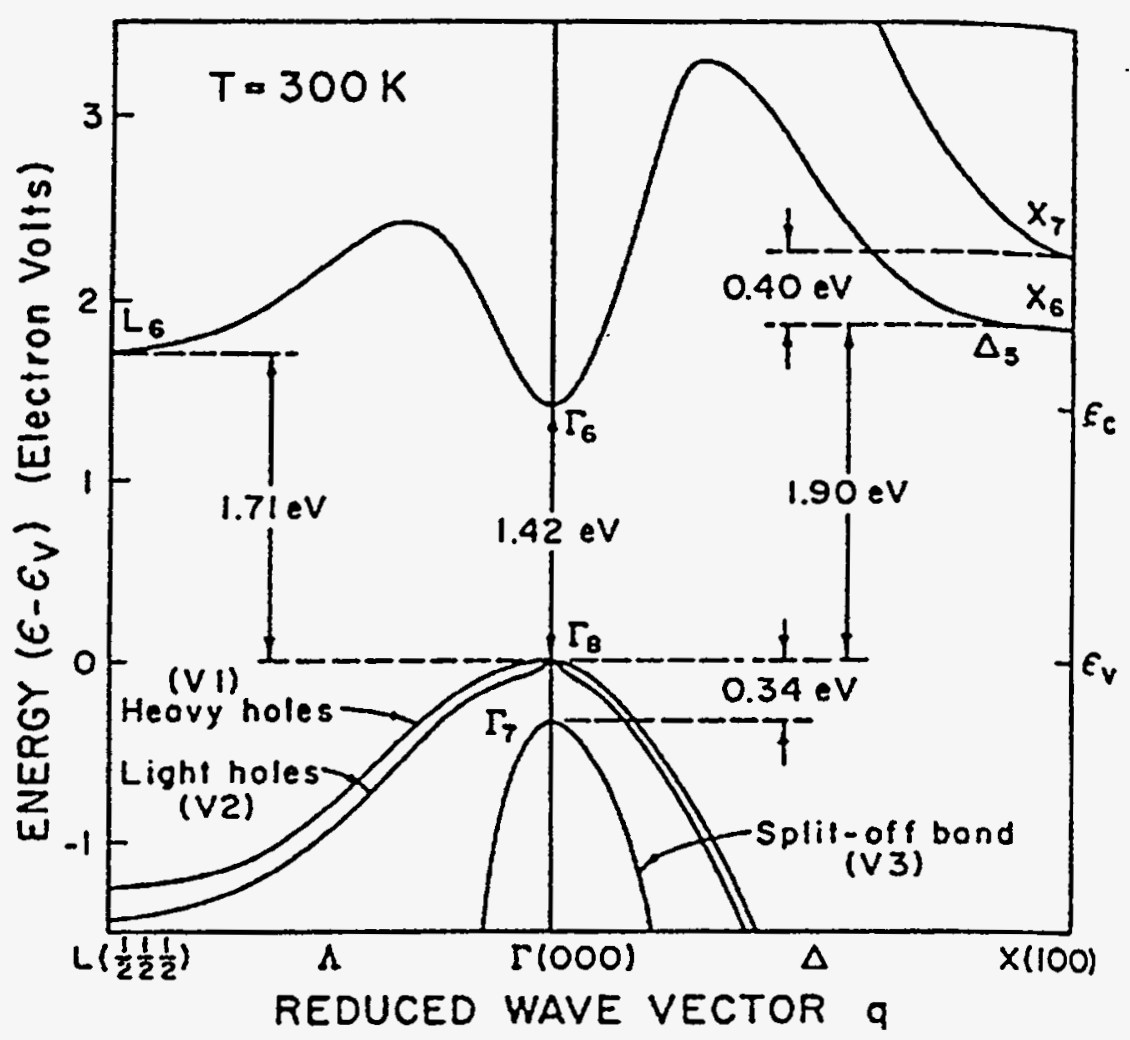

Figure 2.2: Energy band diagram for GaAs.[9] Note the direct bandgap: the bottom of the conduction band coincides with the top of the valence band at $\mathrm{k}=0$ ( $\Gamma$ point $)$.

bands, known as the heavy-hole (HH) and light-hole (LH) bands, are degenerate at the maxima ( $\Gamma$ point). The third band is separated by the spin-orbit splitting energy $\left(\Delta_{s o}=\right.$ $0.34 \mathrm{eV}$ at $\left.T=300^{\circ} \mathrm{K}\right)$. The bottoms of the conduction band, on the other hand, appear along the [111] direction $(L)$, along the [100] direction $(X)$, and at $\mathbf{k}=0(\Gamma)$. The conduction band minimum in GaAs occurs at the $\Gamma$ point and it has only spin degeneracy.[8][9]

\section{Effective Mass of Electrons and Holes in Semiconductors}

The effective mass of electrons and holes in semiconductors is derived from the acceleration they experience in the lattice under the influence of an electric field. At $k=0$ 
there is only one value of the effective mass for electrons in the conduction band and another single value of effective mass for holes in the valence band. The effective mass is obtained from the classical kinetic energy equation and is given by

$$
\frac{1}{m^{*}} \equiv \frac{1}{\hbar^{2}} \frac{\partial^{2} \varepsilon(k)}{\partial k^{2}}
$$

where $\mathbf{p}=\hbar \mathrm{k} .[8]$ The solution to Eqn. 2.1 for GaAs at the $\Gamma$ point is

$$
\begin{aligned}
& \text { Electrons: } \mathrm{m}^{*}=0.067 \mathrm{~m}_{c} \\
& \text { Holes: } \mathrm{m}^{*}=0.082 \mathrm{~m}_{\mathrm{e}}
\end{aligned}
$$

\section{Bandgap Energy Dependence on Temperature}

The bandgap energy in semiconductors has a very strong temperature dependence. This dependence can greatly affect the photoemission properties of these materials. The empirical dependence of bandgap energy on temperature is given by

$$
E_{8}(T)=E_{8}(0)-\frac{\alpha T^{2}}{T+\beta}
$$

where $T$ is the temperature of the material and $\alpha$ and $\beta$ are empirical constants. The values of these constants for GaAs are: $E_{g}(0)=1.519 \mathrm{eV}, \alpha=5.405 \times 10^{-4}$, and $\beta=204$. [8] Table

\begin{tabular}{|l|l|l|}
\hline$T\left({ }^{\circ} \mathrm{K}\right)$ & $E_{g}(\mathrm{eV})$ & $\lambda(\mathrm{nm})$ \\
\hline 300 & 1.422 & 871.9 \\
\hline 275 & 1.433 & 865.2 \\
\hline 200 & 1.465 & 846.3 \\
\hline 150 & 1.485 & 834.9 \\
\hline 100 & 1.501 & 826.0 \\
\hline
\end{tabular}

Table 2.2: Temperature dependence of the intrinsic GaAs bandgap energy. 
2.2 shows some of $E_{\mathrm{g}}$ values for temperatures which are achievable in the GTL. The wavelength $\lambda$ represents the wavelength of a photon of energy $E_{z}$ (i.e. a photon capable of producing a direct bandgap transition).

Large amounts of impurities (i.e. heavy doping concentration) tend to reduce the semiconductor bandgap. However, since the doping concentration remained constant throughout the experiments in this thesis, this phenomenon will not be discussed here. The interested reader should look at references [8][11].

\subsubsection{Carrier Concentration [8]}

Thermal agitation in semiconductors will excite electrons to the conduction band and leave an equal number of holes in the valence band. The excited charge is known as intrinsic carrier concentration and for intrinsic semiconductors (i.e. those with no impurities) it is given by

$$
n_{i}=n=p
$$

where $n_{i}, n$, and $p$ represent the intrinsic, electron, and hole carrier densities, respectively. The excited electrons will eventually recombine with holes in the valence band in a time scale given by the recombination time $\tau$ while other electrons will be excited to the conduction band in such a way of always leaving, on average, $n_{i}$ constant. The density of electrons in the conduction band is given by the Boltzmann distribution

$$
n=N_{C} \exp \left(-\frac{E_{C}-E_{F}}{k T}\right)
$$

where $N_{C}$ is the effective density of states in the conduction band, $E_{C}$ is the energy at the bottom of the conduction band, $E_{F}$ is the Fermi energy, $k$ is Boltzmann's constant, and $T$ is the absolute temperature of the material. Similarly, the hole density near the top of the valence band is given by 


$$
p=N_{v} \exp \left(-\frac{E_{F}-E_{V}}{k T}\right)
$$

where $N_{V}$ is the effective density of states in the valence band and $E_{V}$ is the energy at the top of the valence band.

The Fermi level is defined as the highest occupied energy level at the absolute zero of temperature and can be obtained by equating Eqns. 2.4 and 2.5

$$
E_{F}=\frac{E_{C}+E_{V}}{2}+\frac{k T}{2} \ln \left(\frac{N_{V}}{N_{c}}\right)
$$

Note that the Fermi level for intrinsic semiconductors is close to the middle of the bandgap.

The intrinsic carrier density can be obtained from Eqns. 2.4, 2.5 and 2.6 and is given by

$$
n_{i}=\sqrt{N_{C} N_{V}} \exp \left(-\frac{E_{g}}{2 k T}\right)
$$

where $E_{g}=E_{C}-E_{V}$ is the bandgap energy. The intrinsic carrier density for GaAs at room temperature is $10^{6} \mathrm{~cm}^{-3}$. This number is much smaller than typical impurity concentrations.

Donor or acceptor doping concentrations introduce impurity energy levels in a semiconductor. An acceptor energy level is considered neutral if it is empty or negative if it is filled by an electron. The energy required to ionize the impurities (i.e. release electrons into the conduction band in the case of donors and create holes in the valence band in the case of acceptors) can be computed approximately in a similar way that the ionization energy of hydrogen is computed. The impurity energy levels for $\mathrm{C}, \mathrm{Be}$ and $\mathrm{Zn}$, three common GaAs acceptor dopants, are given by the sum of the valence band energy plus the ionization energy

C: $E_{A}=E_{V}+0.026$

Be: $E_{A}=E_{V}+0.028$

$\mathrm{Zn}: \mathrm{E}_{\mathrm{A}}=\mathrm{E}_{\mathrm{V}}+0.031$ 
where $E_{A}$ is the energy of the acceptor, $E_{V}$ is the energy at the top of the valence band, and the numerical values are the ionization energies determined experimentally and given in $\mathrm{eV}$. The density of ionized acceptors is given by the Fermi-Dirac distribution equation

$$
N_{A}^{-}=\frac{N_{A}}{1+4 \exp \left(\frac{E_{A}-E_{F}}{k T}\right)}
$$

where $N_{A}$ is the acceptor dopant density and the ' 4 ' represents the double spin degeneracy times the double band degeneracy (heavy hole plus light hole). Note that most impurities are ionized at room temperature $(k T \sim 26 \mathrm{meV})$ or higher. The Fermi level will adjust itself to keep the number of holes equal to the number of electrons plus ionized impurities

$$
p=n+N_{A}^{-}
$$

The mass-action law says that the product

$$
n p=n_{i}^{2}
$$

is a constant independent of the added impurities. It can be shown that by combining Eqns. 2.9 and 2.10, the number of holes is given by

$$
p \approx N_{A}
$$

for $N_{A} \gg n_{i}$. Substituting Eqn. 2.11 into 2.5 yields

$$
E_{F(p-s p e)}=E_{V}+k T \ln \left(\frac{N_{V}}{N_{A}}\right)
$$

The importance of Eqn. 2.12 is that it shows the dependence of the Fermi level on dopant concentration and on temperature. 


\subsubsection{Carrier Transport}

The laser pulse produces a relatively low density of photoexcited electrons in the conduction band. As an example, consider a laser pulse with the following numbers: $2 \mathrm{~ns}$ long, $\pi \mathrm{cm}^{2}$ area, $50 \mu \mathrm{J}$ in energy, and a wavelength of $845 \mathrm{~nm}$ has a density of just $1.1 \times 10^{12}$ photons $/ \mathrm{cm}^{3}$. In a photocathode with a very short active region, the electrons produced by this laser pulse have a very small probability of interacting with each other before being photoemitted. The low density of photoexcited electrons suggests that a random-walk model, where the electron motion is independent of other particles, would be more appropriate than a diffusion model which involves a large number of particles for an extended period of time. However, it has been shown that the statistical ensemble average of the electron density from the random-walk model approximates the diffusion equation after an initial short time (of the order of the electron recombination time).[8] This the diffusion equation can be used to approximate simple cases of electron transport during photoemission.

Electrons which are free to move in the conduction band that experience temperature gradients, electric fields, and gradients in charge density will move as acted on by these forces. The average particle current density due to electric fields and diffusion is given by

$$
\mathbf{J}=n q \mu \mathbf{E}+q D \nabla n
$$

where $n$ is the average charge density, $q$ is the magnitude of the electric charge, $\mu$ is the electron mobility, $\mathbf{E}$ is the electric field, and $D$ is the electron diffusion constant. Particle conservation requires that

$$
\frac{\partial n}{\partial t}=G-\frac{n}{\tau}+\frac{\nabla \cdot \mathbf{J}}{q}
$$

where $G(x)$ is the charge generation rate and $\tau$ is the electron lifetime in the conduction band. Substitution of Eqn. 2.13 into Eqn. 2.14 yields 


$$
\frac{\partial n}{\partial t}=G-\frac{n}{\tau}+\nabla \cdot(n \mu \mathbf{E})+\nabla \cdot(D \nabla n)
$$

For the case of steady-state illumination in one-dimension, and in which there are no electric fields $(E=0)$ or temperature gradients, Eqn. 2.15 reduces to

$$
D \frac{\partial^{2} n}{\partial x^{2}}-\frac{n}{\tau}+G(x)=0
$$

For regions of no generation $(G=0)$, the solution to the average electron density is given by

$$
n(x)=A e^{x / L}+B e^{-x / L}
$$

where $A$ and $B$ are arbitrary constants that satisfy the boundary conditions and $L=\sqrt{D \tau}$ is the electron diffusion length. Morse and Feshbach also define the diffusion length as

$$
L \approx \frac{1}{2} \sqrt{v \tau l}
$$

where $v$ is the mean electron velocity, $\tau$ is its lifetime, and $l$ is its mean free path.[12]

A common type of boundary in diffusion problems is a surface which acts as a sink, i.e. a location where electrons can recombine rapidly with the majority carriers (holes in the case of p-type materials). The average diffusion current reaching a surface at $x=0$ is

$$
J_{x}(0)=q D\left[\frac{\partial n}{\partial x}\right]_{x=0}
$$

which can be written in terms of a conduction current

$$
J_{x}(0)=q n(0) S_{0}
$$


where $S_{0}$ is the surface recombination velocity. If all electrons reaching the plane $x=0$ are recombined, then $S_{0}$ will reach a maximum value given by the average velocity of Maxwellian particles crossing a plane:

$$
S_{\max }=\sqrt{\frac{k T}{2 \pi m^{*}}}
$$

where $m^{*}$ is the effective electron mass.[7] For GaAs at T $=300^{\circ} \mathrm{K}, m^{*}=0.07 m_{\mathrm{e}}$ and $S_{\max }=1.02 \times 10^{7} \mathrm{~cm} / \mathrm{s}$.

The diffusion length and the surface recombination velocity are two important parameters that will be considered in the discussion of charge saturation effects and spin relaxation effects later in this chapter and in Chapter 4.

\subsubsection{Surface Properties}

The semiconductor surface plays a major role in the photoemission process. It is the final stage which determines which electrons, if any at all, will escape. The electronic properties of the surface are determined mainly by the following three mechanisms:

- The space-charge or depletion region which is primarily dependent on the density of surface-states, the doping concentration, and the bandgap energy.

- The drop in the surface potential barrier due to the cesium-oxide layer.

- The drop in the surface potential barrier due to the Schottky effect.

This section will focus on the physical basis of the depletion region and on the Schottky effect. The effect of the cesium-oxide layer on the semiconductor surface will be discussed in section 2.2.1.

\section{The Depletion Region}

The formation of the space-charge depletion region is directly related to the density of surface states. As an example, Fig. 2.3 shows the surface configuration of intrinsic (111) silicon, another zincblende crystal. The periodic potential at the surface of the material is 


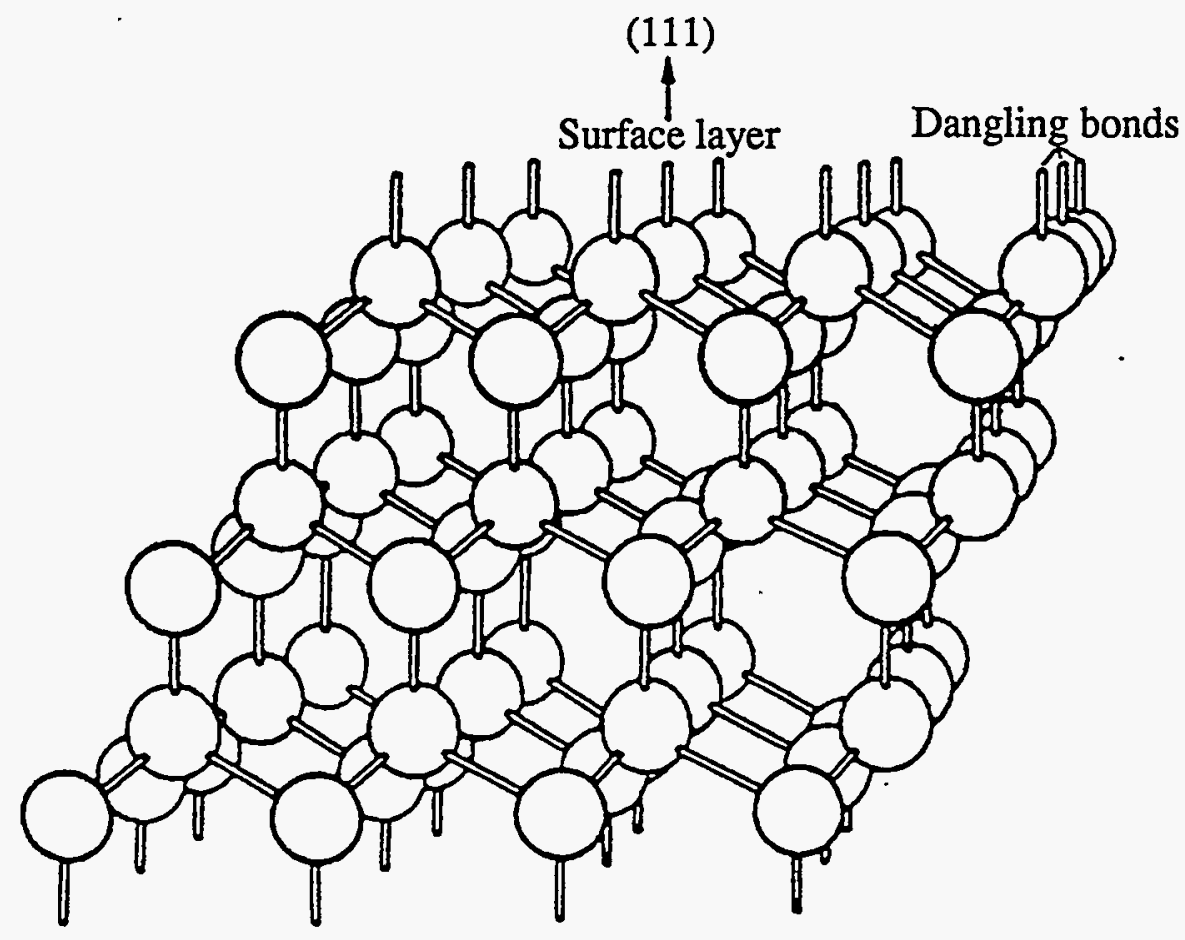

Figure 2.3: Surface configuration of (111) Si.[13] Each surface atom has an unpaired electron whose spin can take any of two states. In other words each surface atom contributes two surface states of which only one half is occupied.

abruptly terminated and the conditions responsible for the bandgap no longer exist. Surface states thus might exist that have energies within the forbidden bandgap. As shown in Fig. 2.3, surface atoms do not have neighbors on the vacuum side to form bonds. These unpaired electrons are known as dangling bonds and they can either give up an electron or accept another electron. If every atom has one dangling bond, then this simple model predicts twice as many states as there are atoms on the surface. Surface neutrality is achieved when half of these surface states are occupied.[13]

The energy distribution of the surface states will depend on the arrangement of the atoms at the surface (i.e. which crystal plane is on the surface), on the atomic composition of the surface (for example, more As than $\mathrm{Ga}$ ), and by relaxation or reconstruction of the surface atoms. The resultant surface energy distribution can be discrete or continuous. If considering a continuous surface state, these states will be filled according to the Fermi- 
Dirac energy distribution and the net surface charge will depend on the position of the Fermi level $E_{F}$. In the case of a neutral surface, $E_{F}$ will split the surface states at midpoint making half of the states occupied and the other half empty. If $E_{F}$ lies below the midpoint, the net surface charge will be positive and if $E_{F}$ lies above the midpoint the net surface charge will be negative.

The addition of impurities to the semiconductor changes the surface potential dramatically. If the impurities are donors, the surface states will gain extra electrons and the surface will become negatively charged. If, on the other hand, the impurities are acceptors, the surface states will donate electrons to the valence band and the surface will become positively charged. The semiconductor region immediate to the surface will thus become positively charged when the impurities are donors and negatively charged when the impurities are acceptors. This region is known both as the depletion region and the spacecharge region.

The Fermi level, which depends on the impurity concentration, will determine the highest filled surface state and thus will also determine the amount of charge in the depletion region.[13] The net charge on the semiconductor surface will be equal and opposite to the net charge in the depletion region. Figure 2.4 shows the depletion region for the three impurity possibilities. Using Gauss' Law, it can be easily shown that for a uniform charge distribution in the depletion region, the potential in the valence band will increase (or decrease) as the square of the charge. The expression for the width of the depletion region is given by

$$
w=\sqrt{\frac{2 \varepsilon_{s} V_{b b}}{q N_{a}}}
$$

where $V_{b b}$ is the band-bending potential, $\varepsilon_{s}$ is the dielectric constant, and $N_{a}$ is the impurity concentration. For GaAs with $N_{a}=5 \times 10^{18} \mathrm{~cm}^{-3}$, the typical value of $w$ is $\sim 90 \AA$. The corresponding surface charge density is $q w N_{a}=4.5 \times 10^{12} \mathrm{e}^{-} / \mathrm{cm}^{2}$.

It has been found that a wide depletion region reduces the probability of escape during photoemission.[10] (This phenomenon will be discussed later in section 2.3.2 and in Chapter 4). For this reason it is desirable to make the depletion region as short as possible. The depletion region can be made shorter by increasing the acceptor doping 


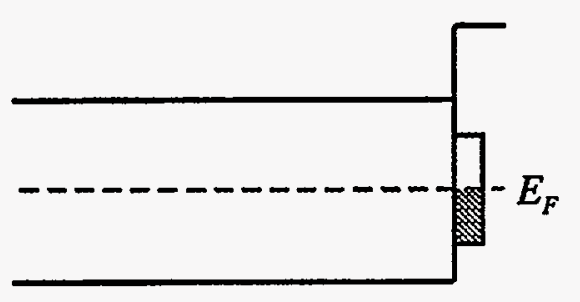

(a)

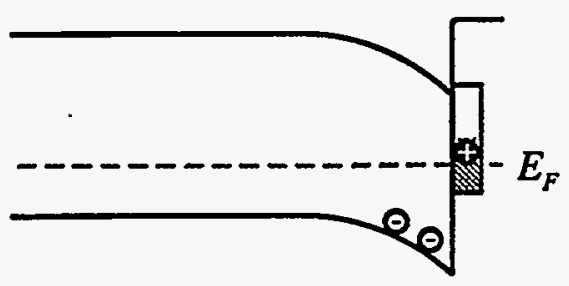

(b)

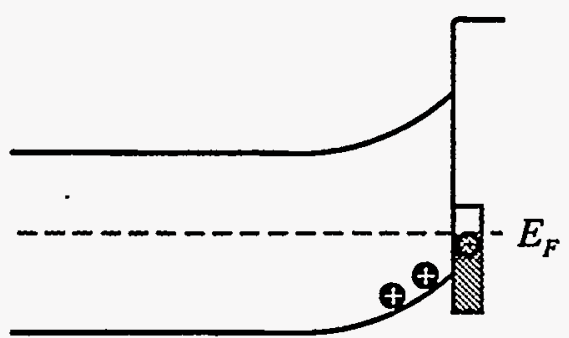

(c)

Figure 2.4: Depletion region in semiconductors: (a) intrinsic - only half of the surface states are occupied leaving the surface neutral of charge, (b) p-doped - surface electrons combine with acceptors in the valence band leaving the surface positively charged, (c) n-doped - electrons from valence band donors combine with surface atoms to leave the surface negatively charged.

concentration. High doping concentrations, however, reduce the diffusion length and thus reduce the $\mathrm{QE} \mathrm{[7][1],} \mathrm{and,} \mathrm{in} \mathrm{addition,} \mathrm{can} \mathrm{cause} \mathrm{spin} \mathrm{relaxation} \mathrm{[14].} \mathrm{For} \mathrm{thick}$ semiconductors, an increased doping concentration will have the net effect of reducing the $\mathrm{QE}$. For thin materials (i.e. those whose active regions are less than the diffusion length of $\sim 1 \mu \mathrm{m}$ ), however, the reduced depletion region due to the increased doping concentration will actually improve the $\mathrm{QE}$. This apparent contradiction will be discussed in Chapter 4 . Another method used to reduce the width of the depletion region is to select the appropriate crystal plane at the surface. The (100) and (110) surfaces have equal amounts of $\mathrm{Ga}$ and As atoms and produce similar band-bending potentials. The (111A) surface is comprised only of $\mathrm{Ga}$ atoms, has the largest $V_{b b}$, and has the worst $\mathrm{QE}$. The (111B) surface is comprised only of As atoms, has the smallest $V_{b b}$, and has the best $\mathrm{QE}$.[10] 
An important note regarding crystal planes at the surface is that they must be treated very carefully in order to preserve their properties. For instance, it has been shown that epitaxial layers which are not originally (110) will facet to give (110) surfaces during most heat-cleaning cycles.[10] This faceting seems to occur when the sample is heated for a few minutes to within $10^{\circ} \mathrm{C}$ of the congruent evaporation point. Heating the sample to $60-70$ ${ }^{\circ} \mathrm{C}$ below the congruent evaporation point seems to leave the semiconductor surface in its original condition. However, the lower heat-cleaning temperature is not as effective at removing surface contaminants. Thus in order to obtain clean surfaces and preserve the original properties of the semiconductor, it is necessary to reduce exposure to contaminants as much as possible and to heat treat the sample for a prolonged time at a safe temperature.

\section{The Schottky Effect}

The surface properties of a semiconductor also change with the application of an electric field. For instance, the well known Schottky effect reduces the surface potential barrier and makes electron emission easier. The two mechanisms in the Schottky effect that affect the potential energy of the electron outside the semiconductor are the external electric field and the image force from charges inside the material. If the vacuum level is arbitrarily set to zero, then these two contributions will reduce the electron potential energy to the following expression

$$
V(x)=-\frac{e^{2}}{16 \pi \varepsilon_{0}} \frac{1}{x}-e F x
$$

where $x$ is the distance from the surface and $F$ is the magnitude of the external electric field.[8] The potential distribution given by Eqn. 2.22 is shown in Fig. 2.5. The maximum value of the potential can be computed by setting the first derivative of Eqn. 2.22 to zero. The difference between the maximum value of the potential and the vacuum level is then given by

$$
\Delta V=e \sqrt{\frac{e}{4 \pi \varepsilon_{0}}} \sqrt{F}=3.7945 \cdot 10^{-5} \sqrt{F(V / m)} \quad \mathrm{eV}
$$




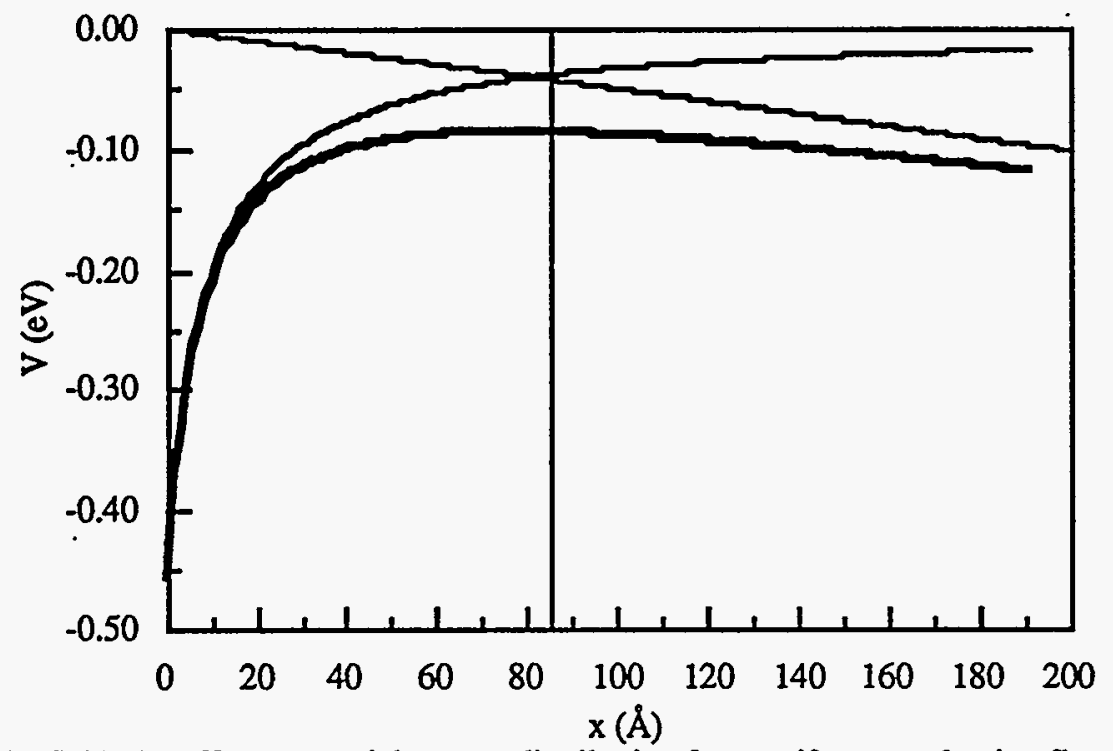

Figure 2.5: The Schottky effect: potential energy distribution for a uniform conducting flat surface under a high electric field. The typical HV bias for a SLAC electron gun is $120 \mathrm{kV}$ with a corresponding electric field on the order of $5 \times 10^{6} \mathrm{~V} / \mathrm{m}$. The maximum potential occurs at $x=85 \AA$ and has a value of $85 \mathrm{meV}$ below the vacuum level.

The maximum potential occurs a distance from the surface given by

$$
x=\sqrt{\frac{e}{16 \pi \varepsilon_{0}}} \frac{1}{\sqrt{F}}=1.897 \cdot 10^{-5} \frac{1}{\sqrt{F(\mathrm{~V} / \mathrm{m})}} \AA
$$

In deriving Eqn. 2.22 it was assumed that the material at $x<0$ is a good conductor. The dielectric constant of GaAs will have a small correction on Eqn. 2.22. The size of the correction will depend on whether the semiconductor has time to polarize as the electron is transmitted. It turns out that the semiconductor has time to polarize and therefore the static dielectric constant can be used in the above computations.[15] Because of the small mass of the electron and because of a very stiff electronic structure of the crystal lattice, the natural frequency of electronic vibration is very high with periods on the order of $10^{-16}$ sec.[16] A free electron traveling for a distance of $50 \AA$ with a thermal velocity of $10^{5} \mathrm{~m} / \mathrm{s}$ will take $5 \times 10^{-14} \mathrm{sec}$, a long time for the atoms in the crystal lattice.

For a dielectric, the potential energy in Eqn. 2.22 has to be modified to 


$$
V(x)=-\frac{e^{2}}{16 \pi \varepsilon_{0}}\left(\frac{\varepsilon-\varepsilon_{0}}{\varepsilon+\varepsilon_{0}}\right) \frac{1}{x}-e F x
$$

The maximum potential and its distance from the surface are then given by

$$
\begin{gathered}
\Delta V=e \sqrt{\frac{e}{4 \pi \varepsilon_{0}}\left(\frac{\varepsilon-\varepsilon_{0}}{\varepsilon+\varepsilon_{0}}\right)} \sqrt{F}=3.4668 \cdot 10^{-5} \sqrt{F(V / m)} \mathrm{eV} \\
x=\sqrt{\frac{e}{16 \pi \varepsilon_{0}}\left(\frac{\varepsilon-\varepsilon_{0}}{\varepsilon+\varepsilon_{0}}\right)} \frac{1}{\sqrt{F}}=1.733 \cdot 10^{-5} \frac{1}{\sqrt{F(\mathrm{~V} / \mathrm{m})}} \AA
\end{gathered}
$$

Note that for cases where $\varepsilon \gg \varepsilon_{0}$, the dielectric behaves similarly to a conductor in that the electric field inside the material becomes very small and the surface-charge density approaches the value of a conducting surface.[16]

\subsection{Photoemission}

The ability of metals when illuminated to emit electrons has been known since the 19th century. In 1905 Einstein put forward the first explanation of this behavior by introducing the concept of the photoelectric effect. During the past century photoemission has had a great deal of influence on society. It has been used not only to study the electronic properties of materials but also as the basis for many electronic devices. A model that consolidates the most relevant aspects of the photoemission process can be found in the Three-Step Photoemission Model by Professor William Spicer. A major breakthrough in the photoemission occurred with the advent of NEA photocathodes. These photocathodes improved the photoemission yield by two orders of magnitude. This section will describe the physical basis for understanding modern photocathodes. In specific, it will focus on the Three-Step Model and on the properties of NEA surfaces. 


\subsubsection{Spicer's Three-Step Photoemission Model}

Photoemission of electrons from a semiconductor into the vacuum has been explained successfully by Professor William Spicer's Three-Step Model.[2] According to this simple model, the photoemission process consists of the following three steps: the photoexitation of electrons into the conduction band, the transport of electrons to the surface, and emission of electrons into the vacuum. Figure 2.6 shows a semiconductor undergoing the three steps of photoemission. The energy distribution of electrons produced deep in the material decreases as the electrons arrive at the surface, causing only electrons with energies greater than the vacuum level to escape.

An important characteristic of a good electron emitter is its ability to have a high optical-absorption coefficient which allows electrons deep in the material to be photoexcited into the conduction band. Figure 2.7 shows that direct bandgap semiconductors, such as GaAs, have the highest absorption coefficients. Their photoemission threshold is characterized by a pronounced knee shape at the bandgap energy. In Fig. 2.7 the knee for GaAs occurs near $1.42 \mathrm{eV}$ or $872 \mathrm{~nm}$. Another characteristic of a good emitter is its low electron affinity, defined as the potential difference between the vacuum level and bottom of the conduction band, which increases the probability of electron emission. Section 2.2 .2 will describe in more detail the advantages

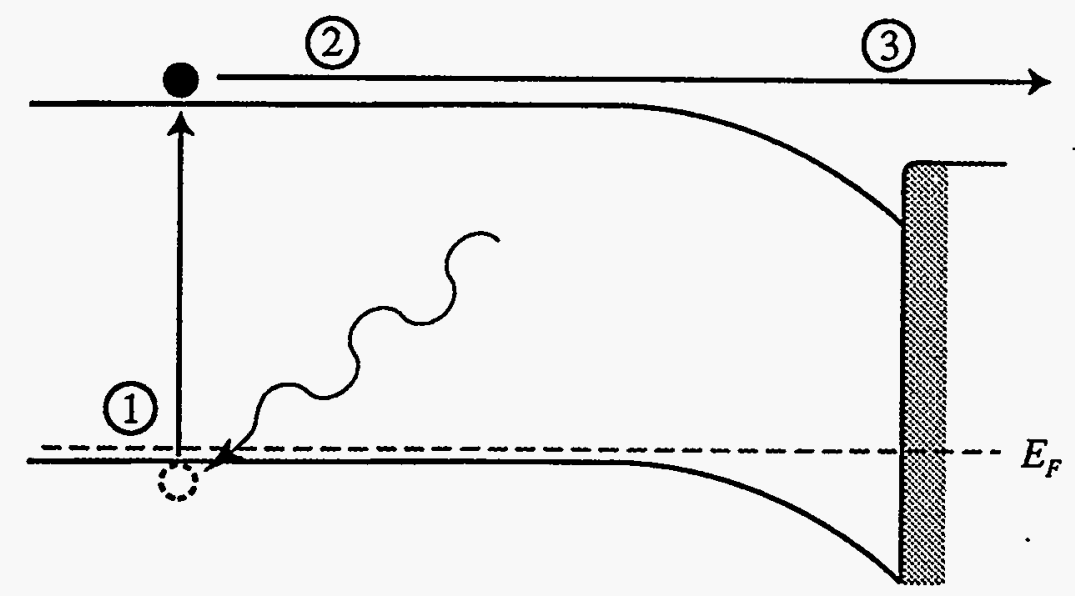

Figure 2.6: Spicer's Three-Step Photoemission Process: 1- photoexcitation of valence electrons into the conduction band (creation of electron-hole pair), 2- transport of electrons to the surface, 3- emission of electrons into the vacuum. 


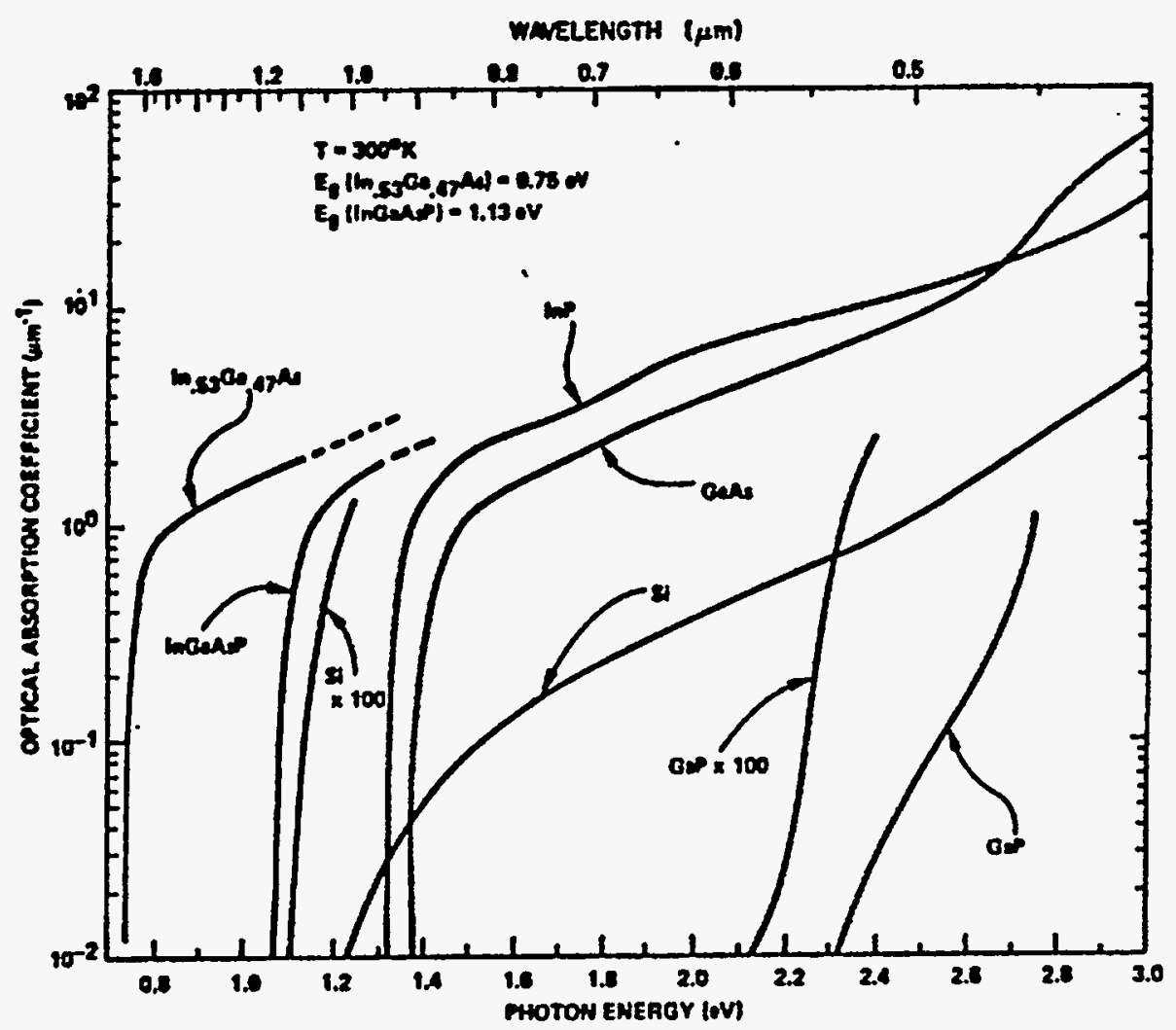

Figure 2.7: Optical absorption coefficient for some NEA semiconductors.[17] The photoemission threshold is characterized by a pronounced knee at the bandgap energy. For GaAs this threshold occurs near $1.42 \mathrm{eV}$.

of lowering the electron affinity. Finally, the work function of good electron emitters, defined as the potential difference between the vacuum level and the Fermi level, should be high in order to reduce the emission of electrons by purely thermal excitation (dark current).

A major problem that metals experience in photoemission is that their Fermi level typically lies above the conduction band minimum. This condition creates an increase in the number of occupied states in the conduction band, causing photoexcited electrons to scatter and to quickly lose their energy. Semiconductors, on the other hand, have a mostly empty conduction band where photoexcited electrons experience minimum electronelectron scattering. Furthermore, some semiconductors have bandgaps large enough that thermionic excitation from the valence band to the conduction band is practically nonexistent at room temperature. 
The most valuable property of p-type III-V semiconductors is their ability to obtain NEA by adding a monolayer of cesium-oxide to the surface. The vacuum level of an NEA semiconductor lies below the conduction band minimum so that electrons can escape practically unscathed. Again, this NEA property will be discussed in more detail in section 2.2.2.

The most common method to quantify the effectiveness of a photocathode is by measuring its $\mathrm{QE}$. The $\mathrm{QE}$ of a photocathode is defined as the ratio of emitted electrons to incident photons. Professor Spicer derived the basic $\mathrm{QE}$ equation for semiconductors. In this derivation the material is assumed to be an infinite half-space with the surface at $x=0$ and photons impinging from the left. The $\mathrm{QE}$ equation is given by

$$
\mathrm{QE}=(1-R) \frac{\frac{\alpha_{P E}}{\alpha} P_{E}}{1+\frac{l_{a}}{L}} \int_{0}^{\infty} e^{-\alpha x} e^{-x / L} d x
$$

where $R$ is light reflectivity from the surface of the solid, $\alpha_{P E}$ is the absorption coefficient for electrons excited above the vacuum level, $\alpha$ is the absorption coefficient for the semiconductor, $P_{E}$ is the probability of escape of electrons reaching the surface, $l_{a}(=1 / \alpha)$ is the photon absorption length, and $L$ is the electron diffusion length.[2] The ratio $\alpha_{P E} / \alpha$ is the fraction of electrons photoexcited above the vacuum level while $l_{a} / L$ gives the ratio of absorption length to diffusion length. All of the variables in Eqn. 2.28 depend on the wavelength of illumination, the temperature of the material, and the doping concentration. For a uniform half-infinite material, Eqn. 2.28 can be simplified into

$$
\mathrm{QE}=(1-R) \frac{\frac{\alpha_{P E}}{\alpha} P_{E}}{1+\frac{l_{a}}{L}}
$$

Equation 2.29 is very useful because it describes the photoemission procèss as a simple function of the underlying variables of the material. For instance, Eqn. 2.29 says that the number of emitted electrons is proportional to each of the following: the light absorbed, the fraction of electrons excited above the vacuum level, and the probability of emission. The 
QE in Eqn. 2.29 is also inversely proportional to the factor $1+l_{\alpha} / L$. This factor implies that the electron emission will decrease for a very small diffusion length $L$ or for a very large absorption length relative to diffusion length (i.e. electrons photoexcited deep in the material will not be able to reach the surface).

\subsubsection{NEA Semiconductors}

The great advantage of III-V semiconductors photocathodes is their ability to obtain NEA. Since electron affinity in a semiconductor is defined as the potential difference between the vacuum level and the conduction band minimum, NEA occurs when the vacuum level lies below the conduction band minimum. The low vacuum level is what makes III-V semiconductors extremely efficient photoemitters. The vacuum level lowering occurs when monolayer quantities of alkali metals of electropositive nature and low ionization potential are deposited on the semiconductor surface. Even lower vacuum levels can be obtained by combining the alkali metals with elements of electronegative nature, such as oxygen or fluorine. The formation of the NEA surface in GaAs is illustrated in Fig. 2.8.

The vacuum level lowering which occurs with the CsF deposits is one of the least

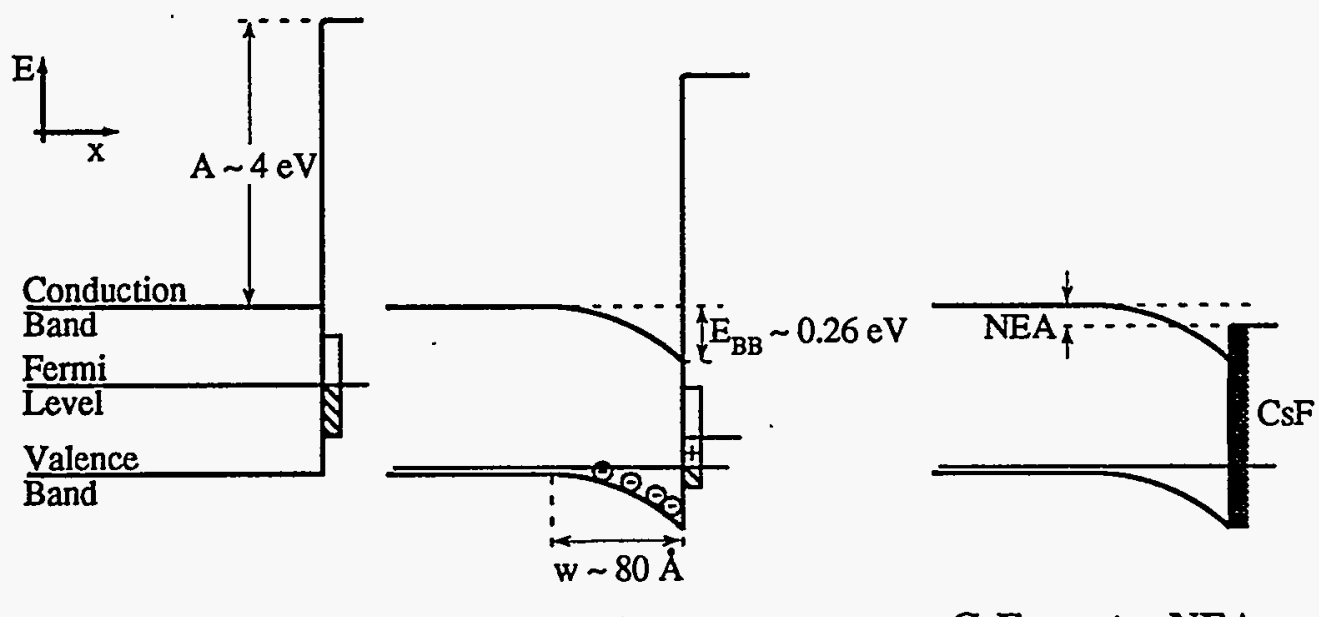

Intrinsic GaAs

p-doped GaAs

CsF creates NEA

Figure 2.8: Formation of the NEA surface. The large electron affinity of intrinsic GaAs makes it impossible for electrons in the bottom of the conduction band to escape. Acceptor impurities help to lower the semiconductor vacuum level. Finally, addition of a cesium-fluoride monolayer helps to lower the vacuum level below the conduction band minimum. 
understood phenomena in the photoemission process. The case of a monolayer or half monolayer of alkali metal on the surface is perhaps best understood using Pauling's electronegative coefficients.[7][17] Among the alkali metals cesium has had the most success in achieving good NEA. Linus Pauling commented that the strong surface binding between a metal and $\mathrm{Cs}$ is a chemical bond with covalent and ionic components. As the Cs atom approaches the metal, it transfers its electron to the metal and becomes ionized. The Cs ion is now an integral part of the metal and its positive charge lowers the work function for metallic electrons. The electrostatic contribution (in $\mathrm{eV}$ 's) to the bond is of the order of $\left(x_{\mathrm{cs}}-x_{\mathrm{m}}\right)^{2}$, where $x_{\mathrm{Cs}}$ and $x_{\mathrm{m}}$ are Pauling's electronegativity values for $\mathrm{Cs}$ and the metal, respectively. Pauling defines the electronegativity of an atom as an average of its ionization potential and its electron affinity

$$
x=0.18(I+A)
$$

which can also be computed by

$$
x=0.44 \phi-0.15
$$

where $\phi$ is the work function of the atom. The ionicity of the bond is given by

$$
1-\exp \left\{-\frac{\left(x_{A}-x_{B}\right)^{2}}{4}\right\}
$$

The electronegativities of $\mathrm{Cs}, \mathrm{Ga}$, and $\mathrm{As}$ are $x_{\mathrm{Cs}}=0.7, x_{\mathrm{Gs}}=1.6$ and $x_{\mathrm{As}}=2.0$, respectively. For the Ga-Cs and As-Cs combinations, the ionicities are 0.19 and 0.35 . This difference in ionicities is probably responsible for the (111A) GaAs (Ga terminated) work function being lower than the (111B) GaAs (As terminated) work function.[7] 


\subsection{Polarized Electrons}

Electron beams with a net spin state (either positive or negative when measured with respect to their axis of travel) are being used more frequently to probe angular momentum properties in matter. As it has been mentioned, some of the most common sources of polarized-electron beams are III-V semiconductors. Their orderly band structure allows scientists to select electrons with a specific spin state. Figure 2.9 (a) shows that by illuminating a $\mathrm{GaAs}$ crystal with polarized light, the resulting electromagnetic transitions can yield electron beam polarizations of $50 \%$. A more detailed description of these transitions is given in Appendix A.

Even higher polarizations can be achieved by modifying the valence band structure of these materials. The basic idea is to break the heavy hole $(\mathrm{HH})-$ light hole $(\mathrm{LH})$

(a) GaAs
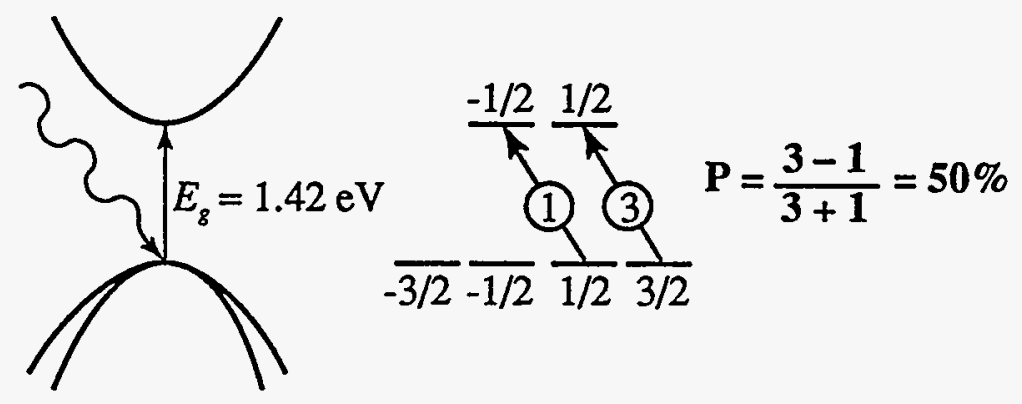

(b) strained GaAs (GaAs on $\operatorname{GaAs}_{0.72} P_{0.28}$ )
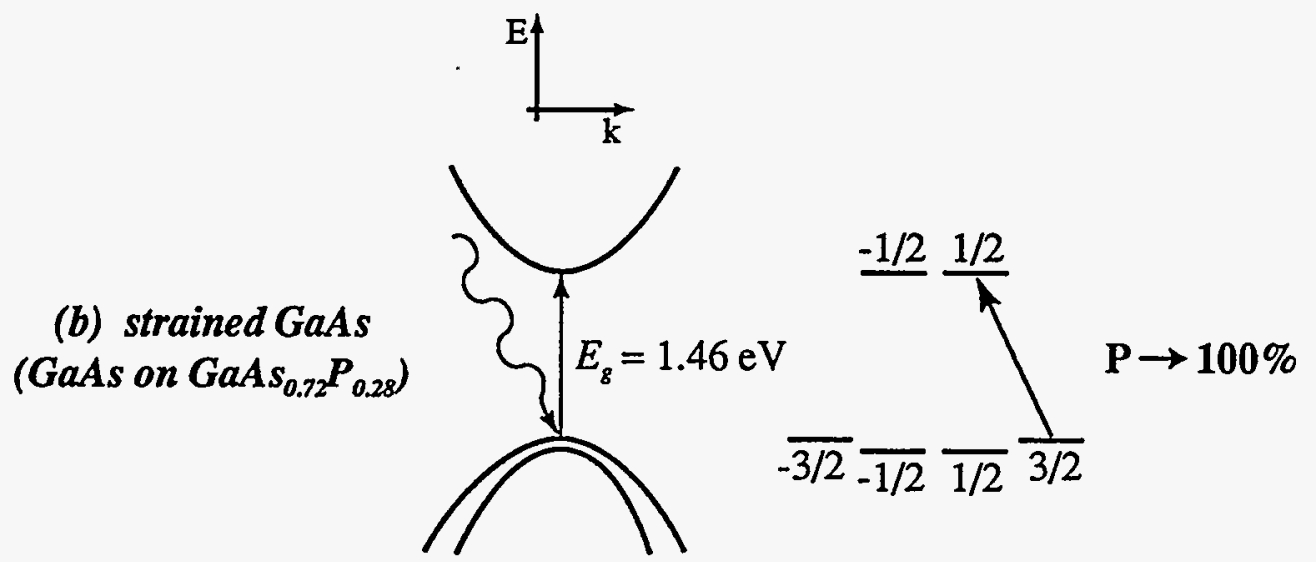

Figure 2.9: Electromagnetic transitions in GaAs. Part (a) shows the electromagnetic transitions in GaAs under left circularly polarized $(L=-1)$ illumination. The numbers inside the circles indicate the relative strength of the transitions. The polarization of the excited electrons approaches $50 \%$. Part (b) shows the electromagnetic transitions in strained $\mathrm{GaAs}$ under left circularly polarized illumination. Note that photons of energy equal to the bandgap create only one type of transition. Thus the polarization approaches $100 \%$. 
degeneracy at $\mathbf{k}=\mathbf{0}$ to allow transitions of only one type of spin state.[4] The broken degeneracy is obtained through biaxial strain resulting from lattice mismatch in heterostructures such as $\mathrm{GaAs}$ grown on $\mathrm{GaAs}_{1-\mathrm{x}} \mathrm{P}_{\mathrm{x}}$. The technique of splitting the valence band at $\mathbf{k}=0$ is shown in Fig 2.9(b). Section 2:3.1 will discuss in more detail the physical basis of the stress process. If completely successful, the stress technique should produce beams with polarizations close to $100 \%$. However, the polarization observed in the laboratory is less than $50 \%$ for unstrained GaAs photocathodes and less than $100 \%$ for strained GaAs photocathodes. Section 2.3.2 will discuss some depolarization process in GaAs photocathodes.

\subsubsection{Strained GaAs}

The energy degeneracy in the valence band arises from the cubic symmetry of the GaAs crystal. Breaking the crystal symmetry through stress deformation will separate the $\mathrm{HH}-$ $\mathrm{LH}$ energy levels. The separate energy levels permit the selection of one type of spin state. The lattice mismatch is defined as $\Delta a / a_{0}=\left(a-a_{0}\right) / a_{0}$ where $a$ and $a_{0}$ are the unstrained lattice sizes of the GaAs layer and the GaAs $s_{1 . x} P_{x}$ substrate, respectively. Figure 2.10 shows that a small mismatch between the top layer and the substrate can be accommodated by tetragonal deformation of the layer lattice. The resulting lattice mismatch normal to the wafer surface is given by $\Delta a_{\perp} / a_{0}=\left(a_{\perp}-a_{0}\right) / a_{0}$ where $a_{\perp}$ is the size of the GaAs lattice normal to the substrate surface. A GaAs layer deformation where $\Delta a_{1} / a_{0}>0$ is said to be in compression while a GaAs layer deformation with $\Delta a_{\perp} / a_{0}<0$ is said to be in tension.

The splitting of the energy levels in GaAs can be computed from the orbital strain Hamiltonian. For a given band at $\mathbf{k}=0$ this Hamiltonian can be written as

$$
\begin{aligned}
H_{\varepsilon}=-a\left(\varepsilon_{x x}+\varepsilon_{y y}+\varepsilon_{z z}\right) & \\
-3 b & {\left[\left(L_{x}^{2}-\frac{1}{3} \mathbf{L}^{2}\right) \varepsilon_{x x}+\left(L_{y}^{2}-\frac{1}{3} L^{2}\right) \varepsilon_{y y}+\left(L_{z}^{2}-\frac{1}{3} L^{2}\right) \varepsilon_{z z}\right] } \\
& -6 \frac{d}{\sqrt{3}}\left[\left\{L_{x} L_{y}\right\} \varepsilon_{x y}+\left\{L_{y} L_{z}\right\} \varepsilon_{y z}+\left\{L_{z} L_{x}\right\} \varepsilon_{z x}\right]
\end{aligned}
$$




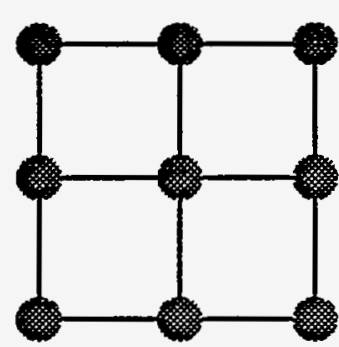

GaAs $a=5.654 \AA$

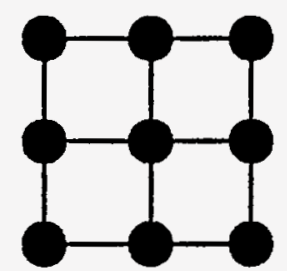

$$
\begin{aligned}
& \mathbf{G a A s}_{0.72} \mathbf{P}_{0.28} \\
& a_{0}=5.596 \AA
\end{aligned}
$$
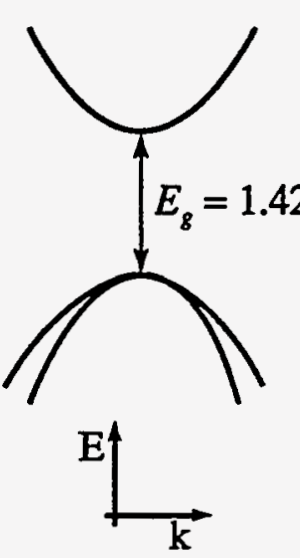
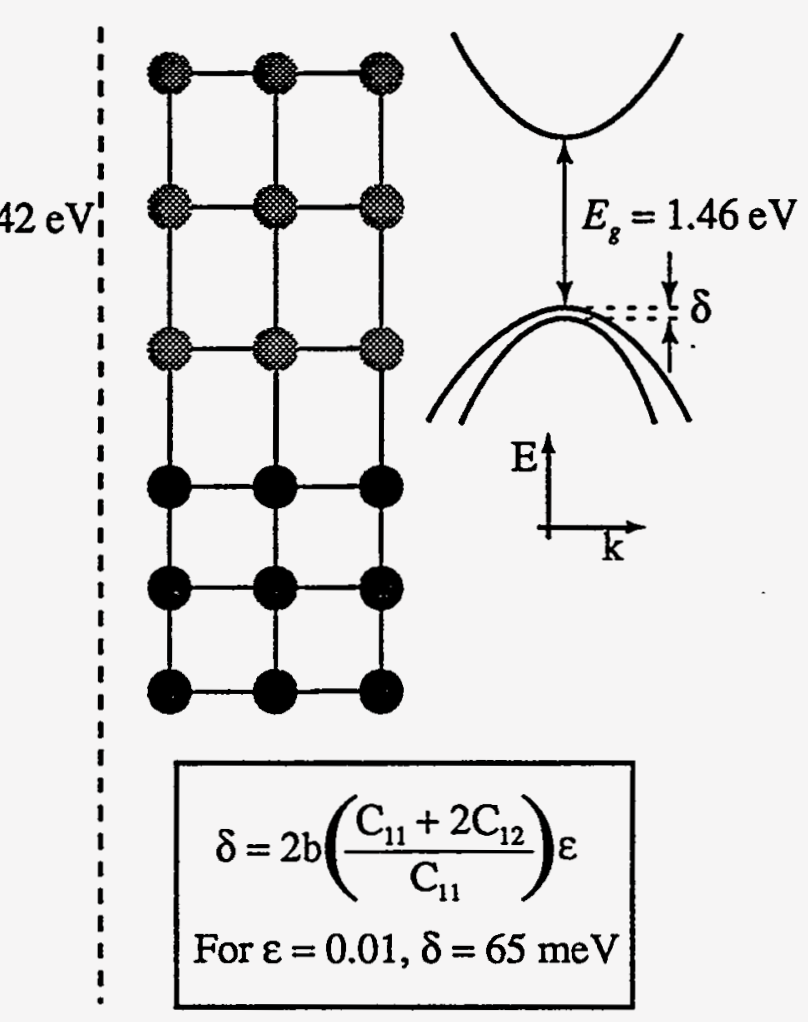

Figure 2.10: Formation of strained GaAs. The figure on the left shows the unstrained crystal lattices for $\mathrm{GaAs}$ and GaAsP. Also shown is the energy-momentum diagram with the HH-LH degeneracy at $\mathbf{k}=0$. The figure on the right shows the compressive strain in GaAs when it is grown on GaAsP. Also shown on the right is the splitting of the valence band energy levels.

where $\varepsilon_{i j}$ represents the components of the strain tensor, $\mathbf{L}$ is the angular momentum operator, and the curly brackets indicate the symmetrized product $\left\{L_{x} L_{y}\right\}=1 / 2\left(L_{x} L_{y}+\right.$ $\left.\mathrm{L}_{\mathrm{y}} \mathrm{L}_{\mathrm{x}}\right)$. The parameters $a, b$, and $d$ are the hydrostatic pressure-deformation potential for a given band, the uniaxial deformation potential for tetragonal strain, and the uniaxial deformation potential for thombohedral strain. When a cubic structure is grown on top of the [001] face of another cubic structure with different lattice constant, the resultant biaxial stress occurs parallel to the [100] and [010] faces. If the elastic strain is defined in terms of the lattice mismatch

$$
\varepsilon=\Delta a / a_{0}
$$

then the strain components are given by 


$$
\begin{gathered}
\varepsilon_{x x}=\varepsilon_{y y}=-\varepsilon, \\
\varepsilon_{z z}=\frac{2 C_{12}}{C_{11}} \varepsilon, \\
\varepsilon_{x y}=\varepsilon_{y z}=\varepsilon_{z x}=0
\end{gathered}
$$

where the elastic strain $\varepsilon$ is defined to be positive for compressive stress and where the $C_{i j}$ represent the elastic stiffness constants. This elastic strain $\varepsilon$ is related to the normal mismatch by

$$
\varepsilon=\frac{\Delta a}{a_{0}}=\frac{C_{11}}{C_{11}+2 C_{12}} \frac{\Delta a_{1}}{a_{0}}
$$

Substituting (2.35) into (2.33) reduces the orbital strain Hamiltonian to

$$
H_{\varepsilon}=2 a \varepsilon\left(\frac{C_{11}-C_{12}}{C_{11}}\right)-3 b \varepsilon\left(\frac{C_{11}+2 C_{12}}{C_{11}}\right)\left(L_{z}^{2}-\frac{1}{3} \mathbf{L}^{2}\right)
$$

where the first term is the shift of the center of gravity of the $P_{3 / 2}$ energy level due to the hydrostatic pressure and where the second term is the linear splitting of the $P_{32}$ level due to the shear deformation from tetragonal strain. The energy eigenvalues for the Hamiltonian in Eqn. 2.37 can be obtained from the unperturbed wave functions of the valence and conduction bands in a zincblende material.[19] The energy differences between the conduction band and the two newly separated HH-LH bands and the spin-orbit band at $\mathbf{k}$ $=0$ are given, to first order in strain, by

$$
\begin{gathered}
\Delta E_{0}(1)=\left[-2 a\left(\frac{C_{11}-C_{12}}{C_{11}}\right)+b\left(\frac{C_{11}+2 C_{12}}{C_{11}}\right)\right] \varepsilon \\
\Delta E_{0}(2)=\left[-2 a\left(\frac{C_{11}-C_{12}}{C_{11}}\right)-b\left(\frac{C_{11}+2 C_{12}}{C_{11}}\right)\right] \varepsilon \\
\Delta\left(E_{0}+\Delta_{0}\right)=-2 a\left(\frac{C_{11}-C_{12}}{C_{11}}\right) \varepsilon
\end{gathered}
$$


The energy difference between the newly split $P_{3 / 2}$ bands is obtained from Eqn. 2.38 and given by

$$
\delta=2 b\left(\frac{C_{11}+2 C_{12}}{C_{11}}\right) \varepsilon
$$

The typical elastic strain obtainable in the laboratory for $\mathrm{GaAs}$ is of the order of $\varepsilon=0.01$. Utilizing the deformation potentials and stiffness constants given in references [18] and [21], the corresponding $\mathrm{HH}-\mathrm{LH}$ energy split is of the order of $\delta=65 \mathrm{meV}$. Most of the information in this section was obtained from references [18], [19], [20], [21] and [4]. In addition, a more detailed description of the stress perturbation can be found in Appendix B.

\subsubsection{Depolarization Mechanisms}

Spin relaxation in the bulk of semiconductors has been studied extensively. The most comprehensive publications on this subject are those of Fishman and Lampel [22] and Pikus and Titkov [5]. These publications discuss the relative strengths of various depolarization mechanisms. For semiconductors with medium to high doping levels, the major depolarization mechanisms near room temperature are:

- the exchange interaction between electrons and holes, or BAP process (after G. L. Bir, A. G. Aronov and G. E. Picus)

- the dynamic narrowing of the magnetic resonance in spin-orbit split-off conduction bands, or DP process (after M. I. Dyakonov and V. I. Perel)

These mechanisms will be discussed in more detail later in this section.

In many studies of spin relaxation mechanisms, the polarization of photoexcited electrons in the bulk of the semiconductor has been measured using the Hanle effect. This effect obtains information on the electron lifetime and spin relaxation time from the depolarization of their luminescence. The degree of circular polarization of the luminescence $P$ is given by 


$$
P=\frac{P_{0}}{1+\frac{\tau}{\tau_{s}}}
$$

where $P_{0}$ is the original electron polarization after photoexcitation (which is determined by the selection rules of optical transitions), $\tau$ is the electron lifetime in the conduction band (for GaAs this number is around $2-3 \times 10^{-10} \mathrm{~s}$ [8]), and $\tau_{s}$ is the spin relaxation time. The derivation of Eqn. 2.40 appears at the end of this section. The spin relaxation time is determined by the depolarization mechanisms present in the material. Equation 2.40 indicates that electrons with a longer lifetime than the spin relaxation time will lose their original spin orientation quickly. In the Hanle technique, a magnetic field $\mathbf{B}$ transverse to the direction of light polarization is used to precess the electron spins. The decrease in the luminescence polarization due to this precesion is given by

$$
P(B)=\frac{P}{1+\Omega_{L}^{2} T_{s}^{2}}
$$

where $\Omega_{L}=g_{c} \mu_{B} B / \hbar$ is the Larmor frequency in the transverse magnetic field and $1 / T_{s}=1$ $/ \tau+1 / \tau_{s}$. The time $T_{s}$ can be obtained from the value of the magnetic field in which the polarization (when there is no field) is halved: $P\left(B_{12}\right)=P(0) / 2$. The electron spin lifetime $\tau$ and the spin relaxation time $\tau_{s}$ are then given by

$$
\begin{gathered}
\tau=\frac{P_{0}}{P(0)} \frac{\hbar}{g_{e} \mu_{B} B_{1 / 2}} \\
\tau_{s}=\frac{P_{0}}{P_{0}-P(0)} \frac{\hbar}{g_{e} \mu_{B} B_{1 / 2}}
\end{gathered}
$$

The Hanle technique is a relatively simple method of finding the electron lifetime and spin relaxation time. In addition, it can probe picosecond-type phenomena without the use of expensive pulse lasers.[5]

There are a few depolarization processes which will not be considered in this work because their spin relaxation rates are two or more orders of magnitude slower than the 
BAP or DP processes. One of them is the EY process (after R. J. Elliot and Y. Yafet) in which the spin-orbit interaction generates non-pure spin states in the conduction band. The resultant mixing of states is proportional to the electron's wavevector $k$. Another depolarization process is the hyperfine coupling or interaction between the electron spin and the nuclear spin. This interaction is very weak and practically unnoticeable in our applications. Finally, recombination radiation has been mentioned as a possible source of lower polarization secondary electrons. These secondary electrons have, in general, less polarization than the electrons initially created by the circularly polarized illumination. The reduced polarization of the recombination radiation is due both to the less than perfect polarization of photoexcited electrons and to dependence of radiation polarization on the angle between the initial orientation of electron spin and the direction of radiation propagation. The process of radiation reabsorption also depends on doping levels. In heavily doped crystals, where strong hole degeneration occurs, the recombination radiation is incapable of creating new electron-hole pairs due to the fact that the interband transitions start at higher valence band energies: $\hbar \omega=\mathrm{E}_{\mathrm{g}}-\mathrm{E}_{\mathrm{F}}\left(\mathrm{m}_{\mathrm{h}} / \mathrm{m}_{\mathrm{c}}+1\right)$.[5] In low or mediumdoped crystals and/or at high intensity illumination, hole degeneration might not exist and optical reabsorption may play a significant role in depolarization.

In the BAP process the exchange interaction between electrons and holes is described by the Hamiltonian

$$
H_{\text {exch }}=A_{e x c h} V \mathbf{J} \cdot \mathbf{S} \delta(\mathbf{r})
$$

where $A_{\text {exch }}$ is proportional to the exchange coupling between the periodic part of the conduction and valence wave functions, $\mathbf{J}$ is the angular momentum operator of the hole, $\mathbf{S}$ is the electron spin, $\mathbf{r}$ is the position of the electron relative to the hole, and $V$ is the normalizing volume. The probability per unit time for a spin flip (or its inverse, the spin relaxation time) can be obtained from Fermi's Golden Rule by finding the sum over all possible initial and final states.[22] The resulting spin-flip rate is given by

$$
\frac{1}{\tau_{S}}=N_{h} \sigma_{B A P} v
$$


where $N_{k}$ is the hole concentration (i.e. acceptor impurity concentration), $\sigma_{B A P}$ can be interpreted as the spin-flip cross section, and $v$ is the electron velocity. Equation 2.44 implies that a high doping concentration and/or higher energy electrons will contribute to a faster spin relaxation rate. Figure 2.11 shows the spin relaxation rate as a function of temperature for high dopant concentration $\mathrm{GaSb}$ and $\mathrm{GaAs}$. Typical spin relaxation rates for the BAP process are between $2-4 \times 10^{10} \mathrm{~s}^{-1}$.

The DP process arises in crystals which lack inversion symmetry (the periodic potential gets altered when $\mathrm{As}$ and $\mathrm{Ga}$ are exchanged). The asymmetry in the crystal lifts the conduction band degeneracy for $\mathbf{k} \neq 0$. The degeneracy holds only for the directions (100) and (111). The splitting of the conduction band is equivalent to an internal magnetic

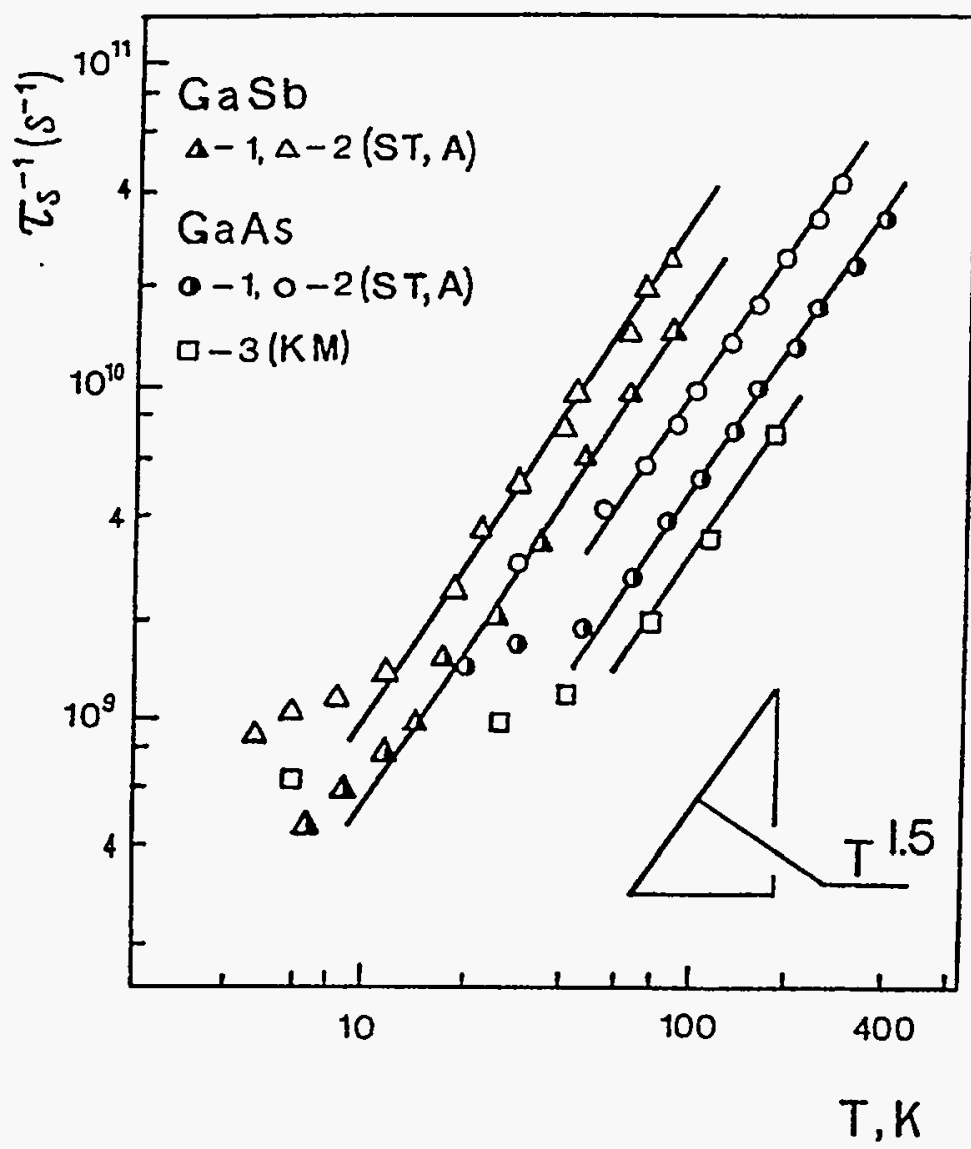

Figure 2.11: Spin relaxation rate as a function of temperature for high dopant concentration $\left(>10^{18} \mathrm{~cm}^{-3}\right)$ GaSb and GaAs.[5] The dopant concentrations are GaSb: (1) $N_{a}=1.8 \times 10^{18} \mathrm{~cm}^{-3}$ and (2) $N_{a}=6.5 \times 10^{18}$ $\mathrm{cm}^{-3}$; and GaAs: $(1,3) N_{a}=5 \times 10^{18} \mathrm{~cm}^{-3}$ and (2) $N_{a}=4 \times 10^{19} \mathrm{~cm}^{-3}$. 
field which in turns is dependent on the magnitude and direction of $k$. The DP process is dominant over the BAP process for doping concentrations $<10^{17} \mathrm{~cm}^{-3}$ and temperatures above $100^{\circ} \mathrm{K}$. A comparison of the BAP and DP processes for low doping concentrations is shown in Fig. 2.12. Typical spin relaxation rates for the DP process are between $4 \times 10^{9}$ $-2 \times 10^{10} \mathrm{~s}^{-1}$. The DP process does not have a large impact on SLAC photocathodes because most photocathodes used in the injector have high doping concentrations.

Not all the proposed depolarization mechanisms occur in the bulk of the material, some take place at the surface. Some authors think that the Cs-O-Cs surface layer causes depolarization during the photoemission process.[23] They propose a simple exponential dependence for this type of depolarization

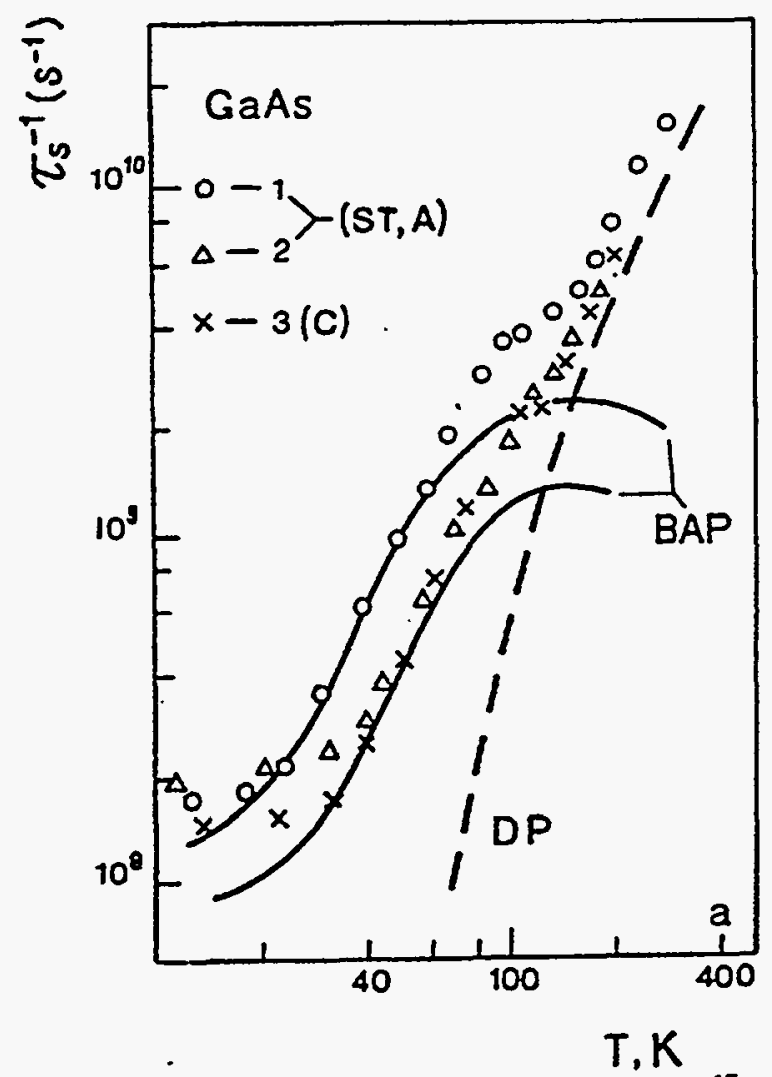

Figure 2.12: Spin relaxation rate as a function of temperature in low $\left(-10^{17} \mathrm{~cm}^{-3}\right)$ doped GaAs.[5] The dopant concentrations are: (1) $N_{a}=3.5 \times 10^{17} \mathrm{~cm}^{-3}$ and $(2,3) N_{a}=2.2 \times 10^{17} \mathrm{~cm}^{-3}$. The solid and dashed lines are theoretical dependencies for the BAP and DP mechanisms, respectively. The points indicate the experimental results. Note that at high temperatures the DP process becomes the dominant depolarization mechanism. 


$$
P(n)=P_{\text {Bulk }} \exp (-\sigma n)
$$

where $P_{B u k}$ is the polarization of electrons just before they escape, $\sigma$ is the exchange scattering cross-section, and $n$ is the number of scatterers per $\mathrm{cm}^{2}$. It is believed that the $\mathrm{Cs}_{2} \mathrm{O}$ surface does not have free electron spins but that the ionized Cs atoms from the second coverage act as spin scatterers. Unfortunately, more detailed literature on this depolarization process is lacking.

\section{Derivation of Eqn. (2.40): steady-state polarization in the conduction band}

A photocathode under steady-state illumination will arrive at an equilibrium polarization in the conduction band: $d P / d t=0$. The three rates involved in the polarization state include: the rate of creation of polarization $P_{0}$ by the circularly polarized illumination, the rate at which the equilibrium polarization $P$ recombines to the valence band, and the rate at which the equilibrium polarization disappears due to the spin relaxation effects. The relationship between these three rates is

$$
\frac{d P}{d t}=\frac{P_{0}}{\tau}-\frac{P}{\tau}-\frac{P}{\tau_{s}}=0
$$

Equation 2.40 is thus found when Eqn. 2.46 is solved for $P$.

\subsection{Charge Saturation}

The electron sources at SLAC must produce large current densities which are required by the high-energy physics experiments. The maximum charge obtainable from GaAs photocathodes is determined by both the effects of the space-charge limit and of the semiconductor charge limit. The space-charge limit effect assumes that an infinite number of electrons are available and that the maximum current density is determined by the electric field characteristics of the extractor. In the semiconductor charge limit (from here on 
charge limit or CL) the maximum current density is determined by the photoemission characteristics of the material. Both effects are observed in the SLAC electron guns. However, the difficulty of maintaining a high $\mathrm{QE}$ makes the CL the most troublesome of the two effects.

\subsubsection{Space-charge limit}

The maximum current density that can be obtained from a charged-particle source is determined by Child's Law.[24] The derivation of Child's Law assumes two planar electrodes, although curved electrodes can also be used. The cathode electrode is thought of as an infinite source of electrons. The electrodes are held to a potential difference of $V_{0}$ and are separated by a distance $d$ as shown in Fig. 2.13. The maximum current density is given by

$$
j=\frac{\kappa}{d^{2}} V_{0}^{3 / 2}
$$

where $\kappa$ is a proportionality constant, and the quantity $\kappa / d^{2}$ is known as the electrode's perveance.[24] The maximum current density given by Eqn. (2.47) is the current density which gives an electric field of zero at the cathode.

Equation 2.47 indicates that the maximum current density obtainable from a cathode

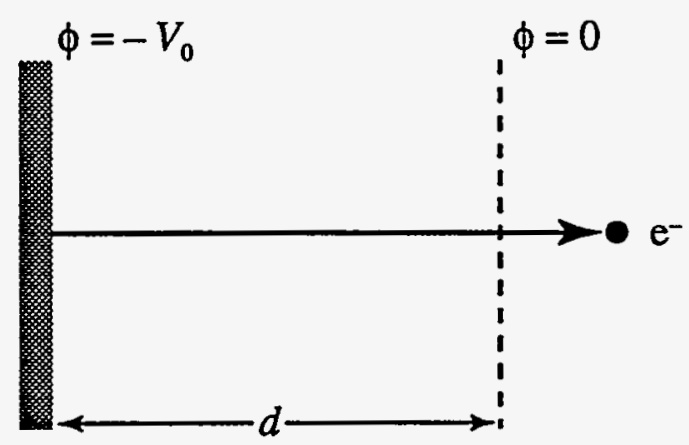

Figure 2.13: Planar electrodes separated by a distance $d$ and held at potentials $\phi=-V_{0}$ and $\phi=0$. 
improves remarkably by increasing the voltage between the electrodes and/or by decreasing their separation. However, practical considerations (mainly dark current produced by field emission) do not permit operating the SLAC guns at extremely high electric fields.[25] Utilizing data from a previous publication [3], the typical maximum current density for SLAC guns operating at a high voltage of $120 \mathrm{kV}$ is on the order of

$$
j \sim 3.4 \times 10^{10} \mathrm{e}^{-} / \mathrm{ns} / \mathrm{cm}^{2}=5.4 \mathrm{~A} / \mathrm{cm}^{2}
$$

This number was obtained by illuminating a $14 \mathrm{~mm}$ diameter $\mathrm{GaAs}$ photocathode with a very high power laser pulse of wavelength $532 \mathrm{~nm}$. The electrons excited by these photons have enough energy to overcome the semiconductor's work function and escape easily. Thus, in this case the limiting factor on the maximum current density was the space-charge characteristics of the gun and not the photoemission limits of the photocathode.

\subsubsection{Charge Limit}

Semiconductor photocathodes are not the perfect sources of electrons assumed in the derivation of Child's Law. On many occasions the maximum charge obtainable from a photocathode is determined by its photoemission characteristics (CL effect) and not by the space-charge characteristics of the gun.[3] (This is particularly the case during the production of polarized beams where it is necessary to illuminate the photocathode with light of energy close to the bandgap energy.)

Very little is known about the CL phenomenon. Some of the few publications that discuss this topic include Woods et. al., "Observation of a Charge Limit for Semiconductor Photocathodes," Herrera and Spicer's "Physics of High Intensity Nanosecond Electron Source," and Alley et al., "The Stanford Linear Accelerator Polarized Electron Source."[3][26][27] Since a large part of this thesis consisted of investigating the CL phenomenon and its effects on the beam polarization, the $\mathrm{CL}$ will be discussed in detail in the experimental sections 4.4 and 4.5 . 


\section{Chapter 3}

\section{The Gun Test Laboratory}

The SLAC Gun Test Laboratory (GTL) was built to develop and study sources of polarized electrons for injection into high-energy linear accelerators. The GTL has a replica of the first few meters of the SLAC injector beamline. The facility includes a high-voltage (HV) electrostatic gun with a loadlock system for easy photocathode exchange under vacuum, a Nd:YAG-pumped pulsed Ti:Sapphire tunable laser, an electron beamline with beam monitoring capabilities, and a Mott polarimeter. Figure 3.1 shows a GTL layout plan including all the beamline components. The GTL has proven very useful for testing charge production, beam transport, $\mathrm{HV}$ processing, injector reliability (including gun diagnostics), photocathode preparation, and the polarization of new photocathodes.

The HV electrostatic gun was designed to produce very intense space-charge limited beams with enough energy for efficient injection into the accelerator. The gun cathode currently operates at a $\mathrm{HV}$ of $-120 \mathrm{kV}$ with respect to ground. However, this $\mathrm{HV}$ makes operation of the photocathode more difficult. The gun must undergo thorough $\mathrm{HV}$ processing to reduce field emission which causes outgasing and contamination of the photocathode.[25] In addition, an ultrahigh vacuum (UHV) of $\sim 1 \times 10^{-11}$ Torr is required to reduce contamination of the extremely sensitive surfaces of the photocathode and HV electrodes.

A loadlock system (similar to those used to manipulate samples between chambers in semiconductor physics) was develop to isolate the gun from the maintenance process of the photocathodes.[28] This isolation has proven beneficial to the HV operation of the gun by protecting the sensitive HV electrodes from contaminants. The loadlock also serves as the transition point for photocathodes between atmosphere and the UHV. The activation of the photocathode in the loadlock chamber involves heat cleaning the surface under vacuum and then applying layers of $\mathrm{Cs}-\mathrm{NF}_{3}$ until the photocathode achieves its maximum photoemission efficiency. (For more details on photocathode preparation see 


\title{
POLARIZATION AND CHARGE LIMIT STUDIES OF STRAINED GaAs PHOTOCATHODES
}

\author{
Pablo J. Sáez
}

SLAC-Report-501

March 1997

Prepared for the Department of Energy

under contract number DE-AC03-76SF00515 


\section{SLAC Gun Test Laboratory}

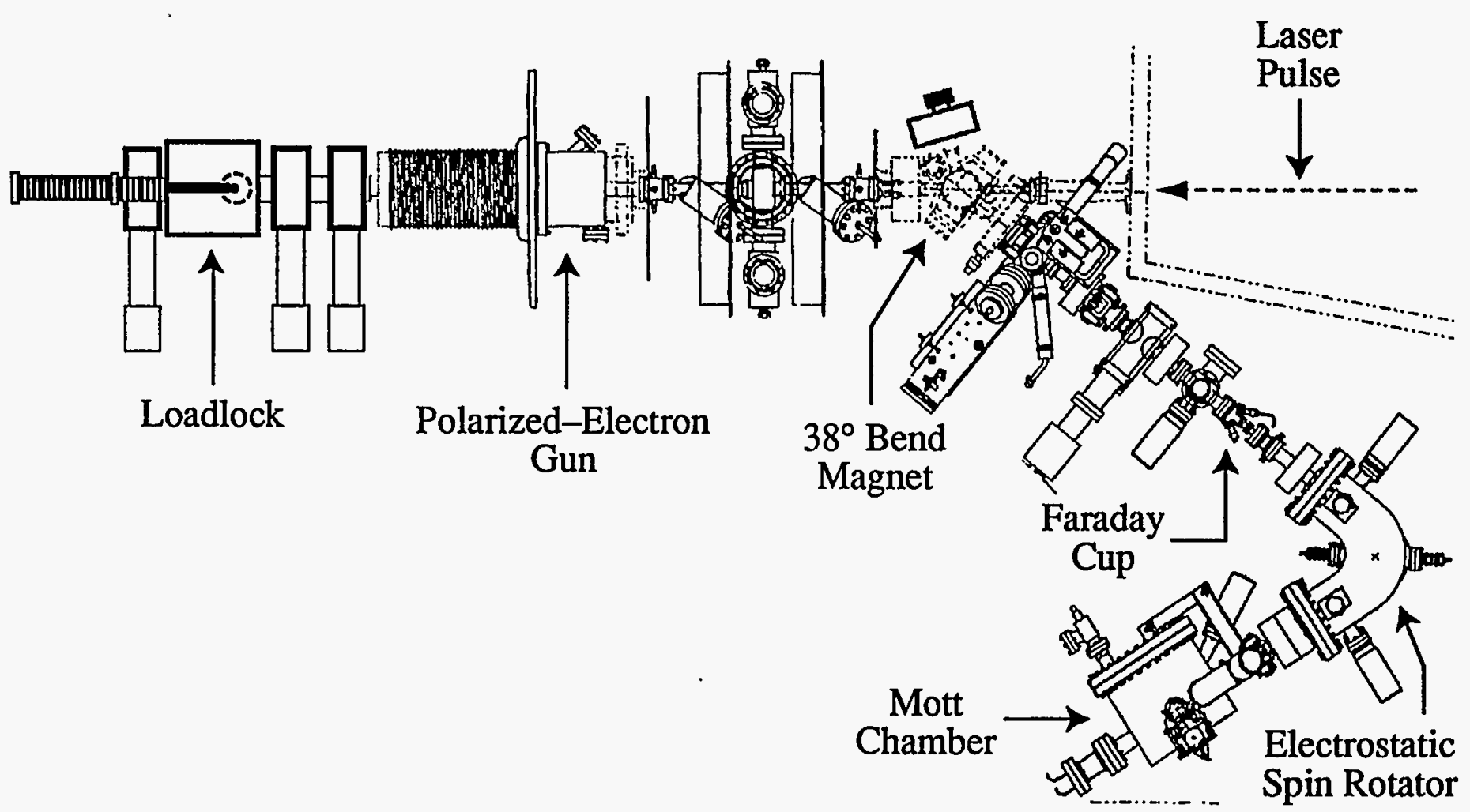

Figure 3.1: Layout of the SLAC Gun Test Laboratory. 
reference [27].) Once activated, the photocathode is transferred from the loadlock activation chamber into the gun.

The injector beamline serves to transport the beam into the accelerator and to monitor beam conditions. The beamline is made up of a series of solenoids which are used as lenses to focus the beam as it spreads due to emittance and non-relativistic space-charge forces. A bend magnet is used to steer the beam towards the Mott beamline. Modeling of the beamline optics was done using PARMELA and CONDOR.[29][30] Beam position monitors (BPMs) located along the beamline measure the beam intensity and its position with respect to the beamline centroid. A gap monitor (GAPM) displays the temporal profile of the electron bunch. The fast Faraday cup (FFC) is also used to measure the temporal profile of the beam, as well as to collimate the large beam intensity by $90 \%$ in order to accommodate the sensitivity of the phototubes in the Mott polarimeter. When the beamline solenoids and steering coils are tuned optimally, the charge transmission between the gun and the FFC can be greater than $95 \%$.

The laser system consists of a Ti:Sapphire laser cavity pumped by a high intensity Nd:YAG laser.[31] The laser wavelength is tunable between $750 \mathrm{~nm}$ and $870 \mathrm{~nm}$. A pulse chopper Pockels-cell system and an intensity control Pockels-cell system produce a temporal Gaussian pulse that is 2 ns full width at half-maximum (FWHM) and a nearGaussian transverse profile. The Ti:Sapphire laser spot size was always adjusted to fully illuminate the photocathode during the experiments described in Chapter 4. A number of low power lasers are used to monitor the $\mathrm{QE}$ in the GTL. These lasers include a HeNe laser with a wavelength of $633 \mathrm{~nm}$ and various $\mathrm{cw}$ diode lasers with wavelengths of 751, 833 and $850 \mathrm{~nm}$. For more information on the laser system see references [31] and [27].

The Mott polarimeter is the latest addition to the GTL. It allows SLAC scientists to measure the polarization of various experimental photocathodes under the same conditions existing at the injector. In particular, the Mott polarimeter can help to investigate how the $\mathrm{CL}$ affects the beam polarization. Section 3.3 discusses the theory of operation of the Mott polarimeter and the design considerations of its various components.

The following sections in this chapter describe the instruments in the GTL which were used to investigate both the polarization and CL characteristics of strained GaAs photocathodes. Special emphasis will be placed on the Mott polarimeter which was built as part of this dissertation. 


\subsection{Gun and Loadlock}

The current design of the SLAC polarized electron gun has evolved from the old thermionic gun designs.[32][33] A cross-section of the gun is shown in Fig. 3.2. The objective of the gun is to produce very intense, low-emittance electron beams that can be efficiently injected into the linear accelerator. The present gun design has achieved these goals very well. However, the arrival of semiconductor photocathodes have made the structural design requirements more complicated. The sensitive photocathode surface must operate in a UHV environment. Therefore the gun cannot have any HV breakdowns or field emission-induced outgasing. In addition, these photocathodes need frequent maintenance such as cesiation of the surface every few days and heat cleaning every few months. The loadlock helps to protect the gun against contaminants by allowing exchange of photocathodes under a UHV environment. The loadlock also permits activation of the photocathodes outside the gun. This section will review the photocathode gun, the HV

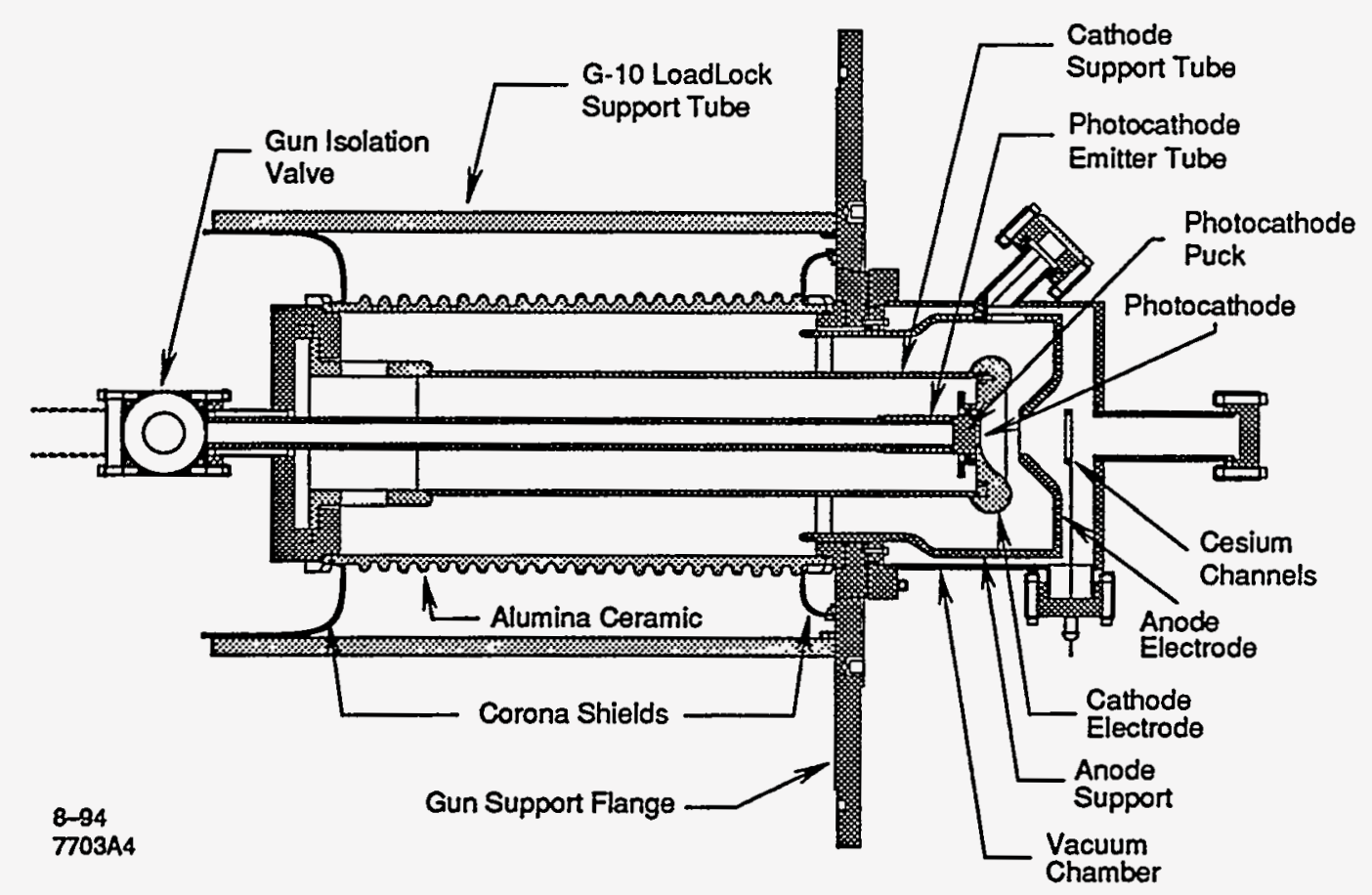

Figure 3.2: Schematic of the SLAC polarized-electron gun. 
processing, and the loadlock system.

The core of the gun consists of a pair of cylindrical Pierce electrodes with a $20 \mathrm{~mm}$ diameter planar semiconductor photocathode. The electrode design was done by Professor Roger Miller who utilized the EGUN simulation program in his design.[34] A crosssection of the electrode design is shown in Fig. 3.3. The curvature of the electrodes produces equipotential surfaces that minimize the emittance from the gun. EGUN calculations for a $-120 \mathrm{kV}$ cathode bias show a field gradient at the photocathode surface of $1.8 \mathrm{MV} / \mathrm{m}$ and a space-charge limited emittance of $35 \mathrm{~mm}-\mathrm{mrad}$.[27] The gun performance would improve with a higher HV for the following reasons: it would yield higher space-charge-limited current densities, and the near-relativistic beam would have smaller radial space-charge forces that tend to blow it up. However, it will be shown later in this section that $\mathrm{HV}$ breakdowns and field emission problems limit the practical $\mathrm{HV}$ bias of the gun to $-120 \mathrm{kV}$. The photocathode is operated in reflection mode, i.e. photoelectrons are emitted from the illuminated surface. The gun is maintained in a UHV environment with a total pressure of $\sim 1 \times 10^{-11}$ Torr. The partial gas pressures are monitored with a residual gas analyzer. A channel cesiator and a $\mathrm{NF}_{3}$ leak valve in front of the electrodes are used to restore NEA to the photocathode after its $Q E$ has deteriorated

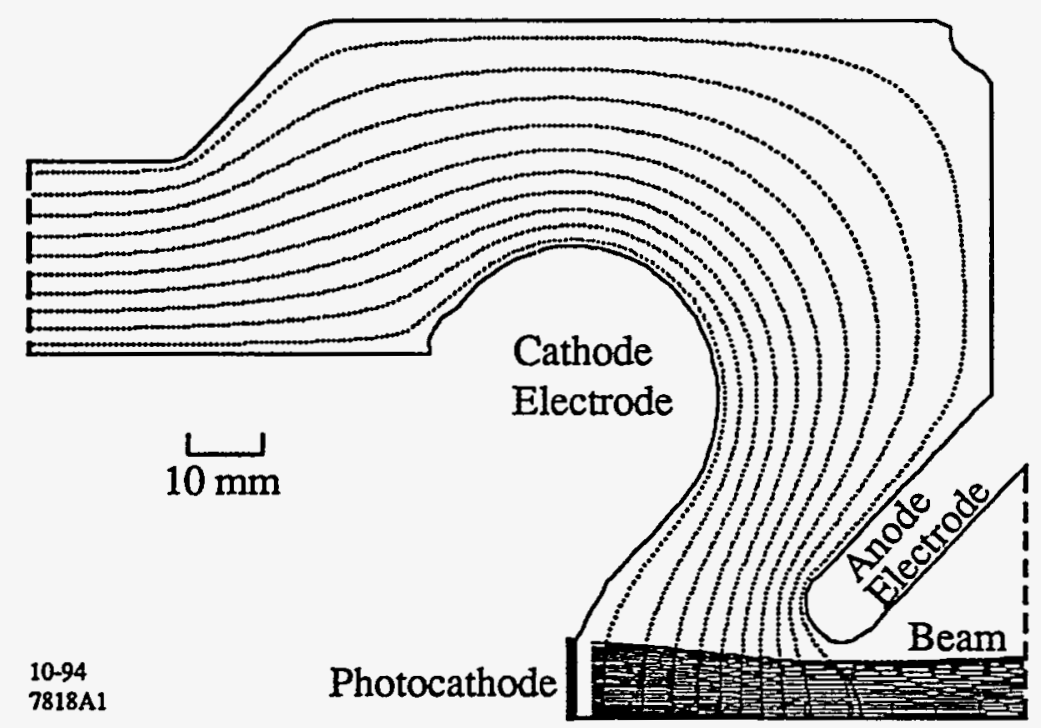

Figure 3.3: Schematic of the Pierce-type electrodes for the SLAC polarized-electron gun. Also shown are the equipotential field lines for $-120 \mathrm{kV}$ cathode bias and the profile of the electron beam density. 
below useful levels. The gun is equipped with a recirculating $\mathrm{N}_{2}$ blow-off cooling system that allows photocathodes to be cooled down to $150^{\circ} \mathrm{K}$. During beam operations the photocathode is maintained at a temperature of $0^{\circ} \mathrm{C}$. Low photocathode temperatures have been associated with longer $\mathrm{QE}$ lifetimes and higher polarizations. However, very low temperatures can result in cryoabsorption of contaminants that can reduce photoemission and can cause HV problems. Finally, an ammeter in series with the gun power supply measures the photocurrent as well as the dark current. This instrument can be used to calibrate the beam current, monitor $\mathrm{QE}$, and diagnose $\mathrm{HV}$ problems. For more details on the photocathode gun see reference [27].

HV plays a central role in the production of high intensity beams. Child's Law states that the space-charge-limited current density of a cathode is directly proportional to $V^{3 / 2}$.[24] Thus a very high $\mathrm{HV}$ is desirable to obtain large current densities and low spacecharge forces. At these voltages, however, HV breakdowns and large dark currents produce a large amount of outgasing. This outgasing quickly contaminates the photocathode surface and destroys the $Q E$. By using $X$-ray sensitive film, it was found that the dark current was produced by field-emitting point sources on the electrodes.[25] Surface analysis of the electrodes found that some of the contaminants included potassium chloride (a common salt found in humans) and cesium. In the past, it was also observed that surfaces made of copper, which is much more reactive than stainless steel, were prone to HV problems. To reduce both the dark current and the probability of a HV discharge, new gun assembly procedures were adopted. The electric field gradients of the electrodes were reduced, the HV surfaces were carefully polished and chemically cleaned, the HV electrodes were fabricated from low carbon content and low inclusion density stainless steel, and very strict clean-room assembly procedures were adopted. In addition, it has been observed that HV problems can be considerably reduced if the gun is maintained in UHV after HV processing. It is possible to process a SLAC gun so that it can run for years at a HV of $-120 \mathrm{kV}$ and with dark currents below $50 \mathrm{nA}$.

The loadlock has become a critical part of the SLAC gun program. It allows the photocathodes to be handled in UHV in much the same way that samples are handled in experimental semiconductor physics. A schematic of the SLAC loadlock system is shown in Fig. 3.4. The main benefits of the loadlock can be summarized as follows:[28]

- isolates the gun UHV from the 'harsh' environment of photocathode activation 
- provides a photocathode activation chamber that is connected directly to the gun

- stores up to four photocathodes in UHV

- provides the transition point for photocathodes between atmosphere and the UHV of the accelerator

- stores the photocathodes during the HV processing of the gun

Having the photocathode activations done outside the gun UHV protects the gur electrodes from contaminants and thus improves the overall HV performance. The loadlock activation chamber has the capability to heat treat the photocathode, apply layers of $\mathrm{Cs}$ and $\mathrm{NF}_{3}$ to its surface, and monitor its QE. Every time the photocathode requires maintenance, these tasks can be performed both in a few hours and right in the accelerator. Finally, the puck tray brings flexibility to the loadlock system. It can carry up to four photocathodes and it can be attached quickly (in less than four hours) to the UHV of the loadlock. The loadlock system has clearly improved the flexibility and reliability of the injector.

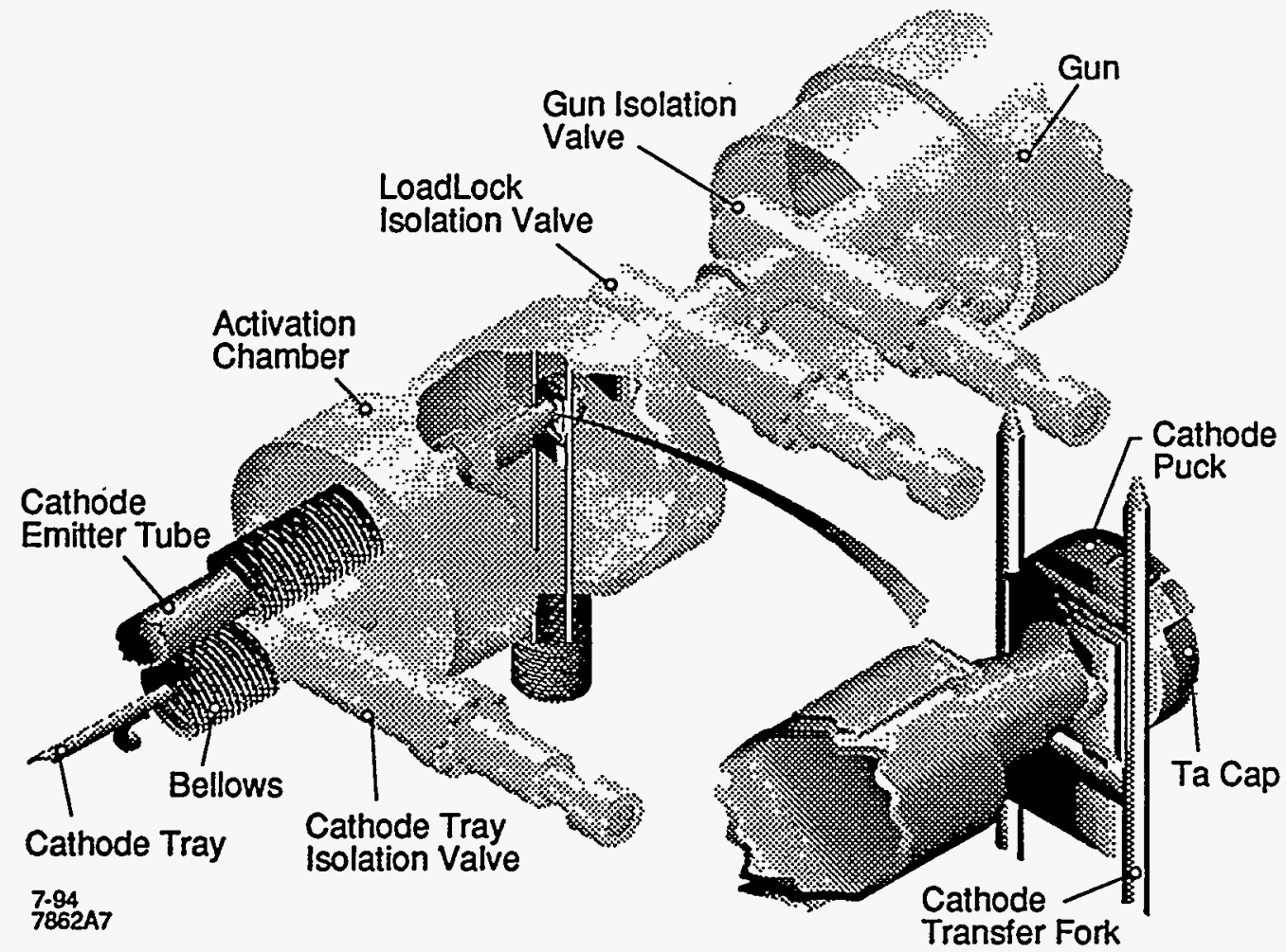

Figure 3.4: Schematic of the SLAC loadlock system. The various valves are used to isolate the activation chamber from the gun and from the puck tray which carries additional photocathodes. 


\subsection{Injector Beamline}

The objective of the beamline is to efficiently transmit the electron beam from the gun into the injector. The arrival of semiconductor photocathodes has increased the imperative to reduce beam interception with the beamline. Such interception, especially near the gun, produces outgasing that can quickly degrade the NEA surface. Beams of up to $10 \mathrm{Amps}$ at a $\mathrm{HV}$ of $-120 \mathrm{kV}$ have been successfully produced with the new photocathodes and transmitted through this beamline.

The electron optics design for the injector beamline was done with the aid of both the EGUN and CONDOR programs.[29][30] A series of solenoid magnets along the beamline control the space-charge of the beam. These magnets are shown in Fig. 3.1. A pair of Helmholtz coils are used to generate axial fields along the beamline to control the transverse beam size. A number of smaller solenoid lenses provide localized fields that help to focus the beam. A $38^{\circ}$ bend magnet is used to steer the beam into the Mott polarimeter. Smaller solenoids with fields perpendicular to the beam axis help to steer in the $\mathrm{X}$ and $\mathrm{Y}$ directions. $\mathrm{X}$-ray sensitive photomultiplier tubes (PMTs) are placed along the
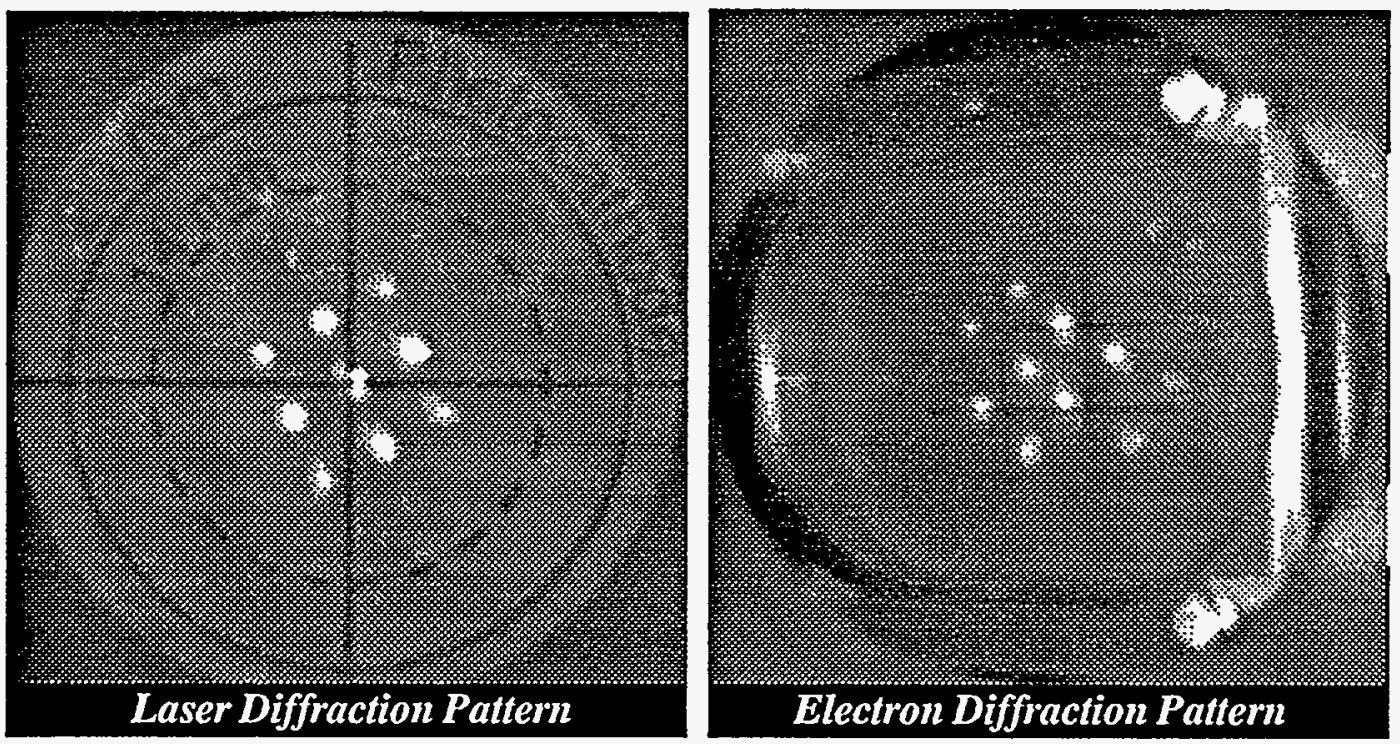

Figure 3.5: Transmission of a diffracted beam pattern in the GTL beamline. The picture on the left shows how the optical diffraction of the laser beam looks on the photocathode. The diffraction pattern was obtained by placing a fine metallic mesh in the laser path. The picture on the right shows the electron pattern on the alignment screen of the FFC. 
beamline to monitor scraping of the beam on the beampipe. Beam loses are reduced by steering the beam in such a way as to minimize PMT signals. The beam transmission between the gun and the FFC for an optimized beamline is greater than $95 \%$. The good transmission properties of the beamline can be seen in Fig. 3.5. The photocathode was illuminated with a diffracted laser beam and the image of the pattern was obtained on the alignment screen of the FFC.

Several beam monitoring instruments help to characterize and monitor the electron bunch. The BPMs can measure the beam intensity as well as the transverse centroid position. The GAPM and FFC display the temporal profile of the electron bunch. A wire scanner gives a cross section of the electron bunch. The FFC has a $2 \mathrm{~mm}$ diameter aperture to collimate the beam. The collimated beam goes into the Mott beamline for polarization studies.

\subsection{Mott Polarimeter Beamline}

The Mott polarimeter beamline is a simple extension of the injector beamline whose purpose is to measure the average polarization of the electron beam produced by the photocathode gun. In Mott scattering the spin-orbit interaction of high-energy electrons impinging on fixed-target nuclei results in an azimuthal scattering asymmetry. From this asymmetry the average beam polarization can be computed. A schematic of the beamline is shown as part of Fig. 3.1. The Mott beamline components include the FFC which collimates most of the intense beam, an electrostatic spin rotator which transforms the longitudinal electron spin to transverse, the Mott chamber with gold foil targets and two detectors, and a series of solenoids and corrector coils to focus and steer the beam.

\subsubsection{Fast Faraday Cup}

The FFC was developed to study the time structure of the electron bunch. Specifically, it was designed to study the time evolution of the $\mathrm{CL}$ in photocathodes. Lowell Klaisner designed the FFC to resolve time structures with a resolution of $100 \mathrm{psec}$.[35] In addition, 
the FFC has a $2 \mathrm{~mm}$ diameter aperture which lets only a small fraction of the beam go on into the Mott polarimeter.

Figure 3.6 shows a schematic of the FFC. A six-way cross supports the various components of the FFC. These components include a translating fluorescent screen used to align the electron beam, a viewing port for the fluorescent screen, a pump-out port for a 45 $\mathrm{l} / \mathrm{s}$ ion pump, and the beam collector cup. One arm of the cross is available to add new instruments for future experiments.

The cup itself is designed to be the center conductor of a coaxial line with the beam pipe being the outer conductor. The characteristic impedance of this line is $12.5 \Omega$. This low impedance produces a voltage of $125 \mathrm{~V}$ for a typical 10 Amps beam. The low impedance of the cup was chosen to limit the voltage, which accelerates secondary electrons away from the cup, and to provide a large aperture of 1.0 " ID for the incident

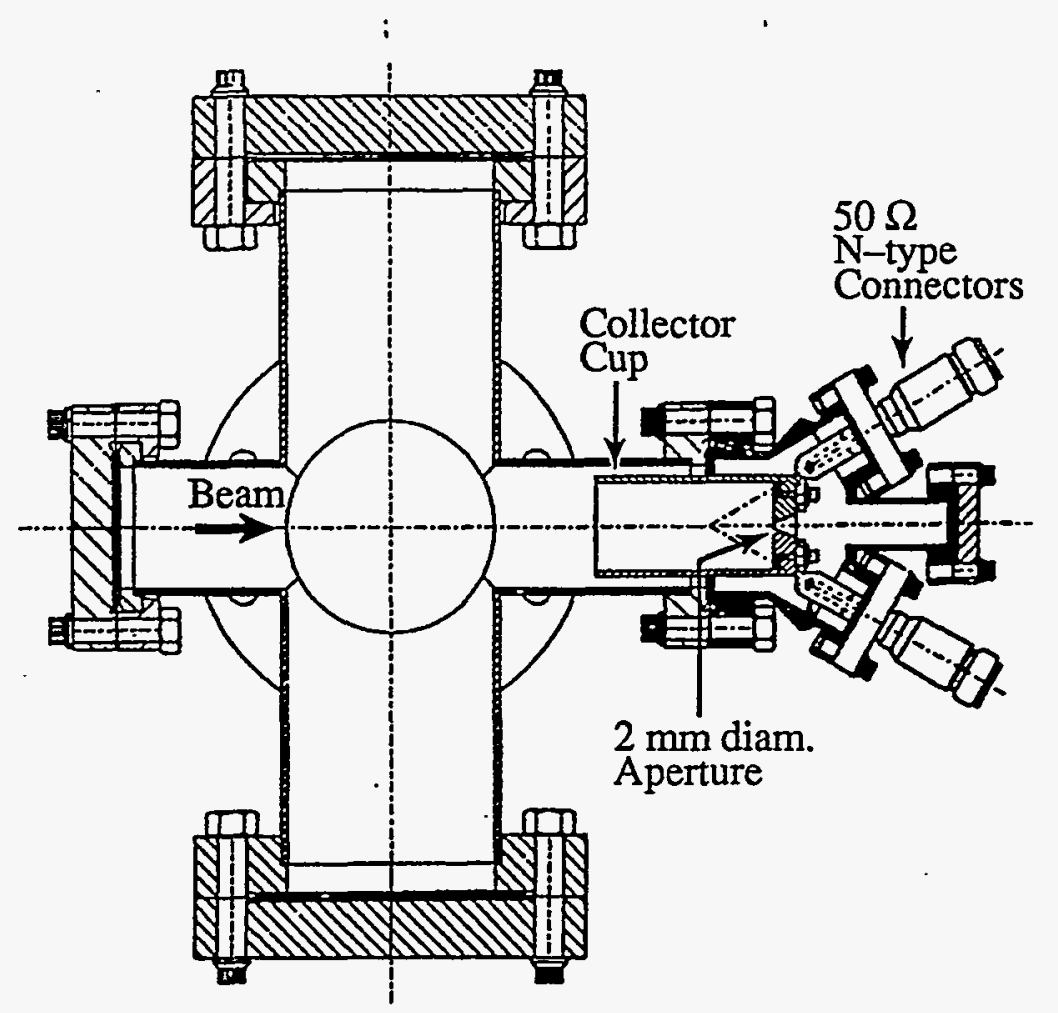

Figure 3.6: Schematic of the fast Faraday cup. The six-way cross supports all the FFC components and leaves one port available for future experiments. The beam enters the cross from the left and is collected in the cup on the right side of the cross. A $45 \mathrm{l} / \mathrm{s}$ ion pump is located on the bottom and an alignment screen is lowered from the top arm of the cross. 
beam. The transmission line is terminated with four parallel $50 \Omega, N$-type connectors which match the line impedance. Three of these connectors are terminated at the cup and the fourth is attached to a Heliax cable which carries the signal to a $250 \mathrm{GHz}$ sampling oscilloscope and to a Gated Analog to Digital Converter (GADC).

The $2 \mathrm{~mm}$ diameter aperture allows a small fraction of the beam to pass on to the polarimeter. The amount of charge transmitted is adjusted by changing the beam spot size on the cup. The aperture has a 0.025 " edge in the $\mathrm{Z}$ direction to minimize the number of scattered electrons passing on down the beamline.

\subsubsection{Spin Rotator}

The asymmetry in Mott scattering arises from the spin-orbit interaction between the electron beam and the target nuclei. The strength of the interaction is greater when the electron spin is perpendicular to the scattering path. The spin rotator transforms the spin orientation of the beam from longitudinal to transverse. The spin transformation is achieved by electrostatically deflecting the beam trajectory by $90^{\circ}$. Since the electric field does not interact with the magnetic moment of the electron, the spin orientation remains intact and thus becomes transverse to the trajectory after the deflection. However, there is an additional precession of the spin for semi-relativistic beams like the ones produced by the SLAC guns. In the rest frame of the spin, the moving electric field looks like a magnetic field which induces a small torque on the magnetic moment of the electron. This section will review the physics of spin transformation, the HV required for circular deflection, and how the spin rotator can be used to calibrate the beam energy.

The spin rotator in the GTL Mott beamline consists of two plates held at equal potential magnitude but opposite polarity. The resulting uniform electric field deflects the beam in a circular trajectory. Figure 3.7 shows an illustration of the electrostatic plates. To determine how much the beam trajectory needs to be deflected in order to obtain a transverse spin orientation, it is necessary to understand how the magnetic moment of the electron is affected by the electric field. For an electron traveling in an electric field, the relativistic expression for the electron spin precession is given by 


$$
\Theta_{\text {prec }}=\left[\frac{g-2}{2}-\frac{1}{\gamma^{2}-1}\right] \frac{\gamma^{2}-1}{\gamma} \Theta_{b e n d}
$$

where $\Theta_{\text {prec }}$ is the spin precession angle, $g$ is the gyromagnetic ratio (Landé factor), $\gamma$ is the Lorentz energy factor $\left(1-\beta^{2}\right)^{-1 / 2}$, and $\Theta_{b e n d}$ is the angle of trajectory deflection in the plane of precession.[36] An outline of the derivation of Eqn. 3.1 is given in Appendix C. The gyromagnetic anomaly $(g-2) / 2$ for electrons and positrons is $1.159652 \times 10^{-3}$. A simpler form of Eqn. 3.1 can be obtained by ignoring the small effect of the gyromagnetic anomaly

$$
\Theta_{\text {prec }} \approx-\frac{\Theta_{\text {bend }}}{\gamma}
$$

Equation 3.2 says that the angle of spin precession lags the angle of trajectory deflection by the Lorentz energy factor. Figure 3.8 shows how the spin rotator accomplishes the $\Theta_{\text {prec }}=$ $90^{\circ}$ spin transformation by electrostatically deflecting the trajectory of a $120 \mathrm{keV}(\gamma=$ 1.2348 ) electron beam by $\Theta_{\text {bend }}=111.2^{\circ}$.

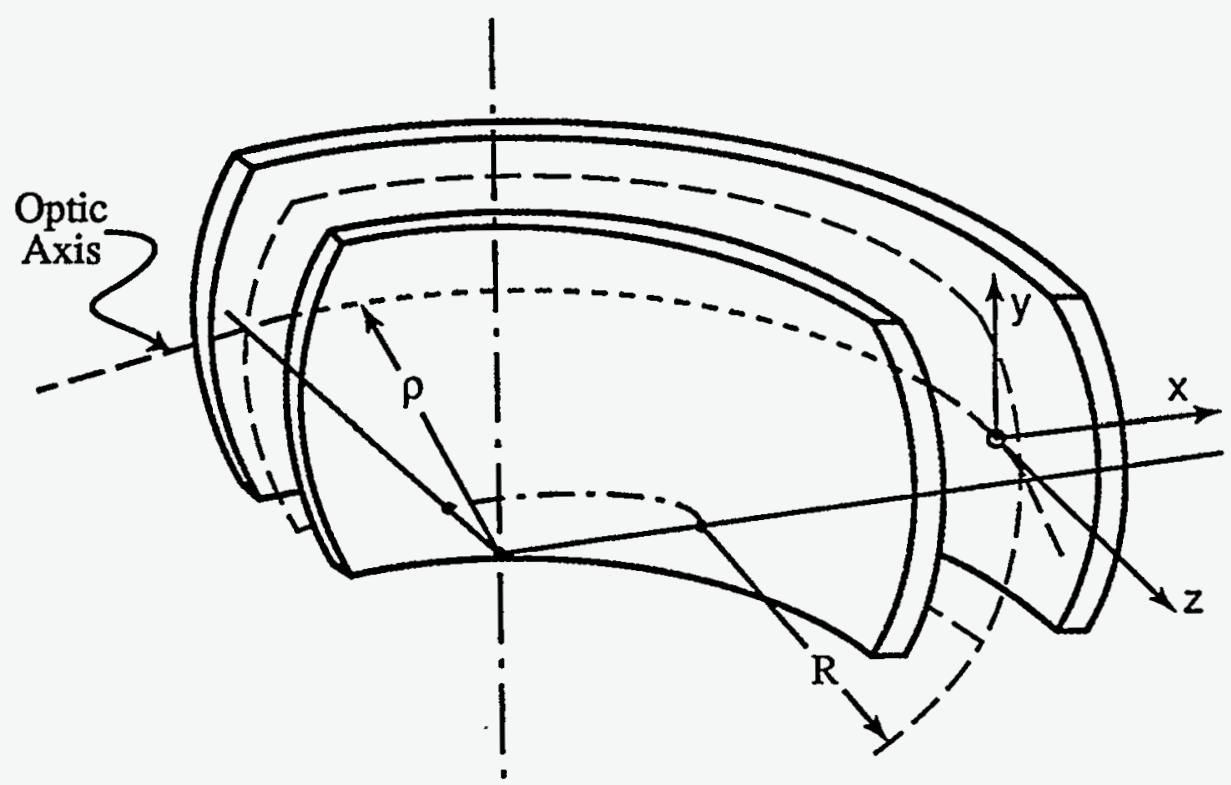

Figure 3.7: The electrostatic spin rotator.[37] After the beam is deflected electrostatically, the spin orientation becomes transverse to the direction of propagation. 
The electric field necessary to deflect a relativistic beam in a circular trajectory of radius $r_{0}$ is given by

$$
e E=\frac{y m v^{2}}{r_{0}}
$$

To a first approximation, the deflecting plates can be thought of as two parallel plates held at equal potential magnitude but opposite polarity and separated by a distance $d$. The resulting electric field is then given by

$$
E \approx \frac{2 V}{d}
$$

where $V$ is the absolute value of the potential of each plate. Utilizing the theory of relativity, the square of the velocity can be expressed as

$$
v^{2}=\frac{\gamma^{2}-1}{\gamma^{2}} c^{2}
$$

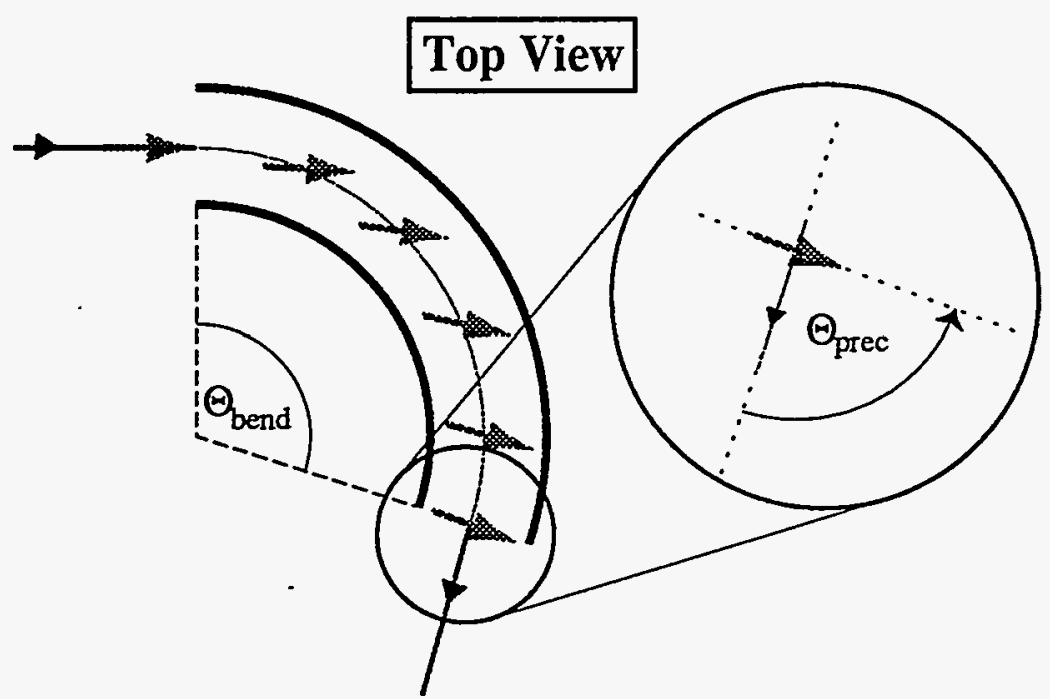

Figure 3.8: Spin rotation in an electrostatic bend. $\theta_{\text {bend }}$ is the angle that the trajectory sweeps under the electrostatic field. $\boldsymbol{\Theta}_{\text {prec }}$ is the angle that the spin has rotated after the deflection is completed. 
A new expression for the electrode voltage is obtained by substituting Eqns. 3.4 and 3.5 into Eqn. 3.3 and solving for $V$

$$
V=\frac{\gamma^{2}-1}{\gamma} \frac{d}{2 r_{0}} \frac{m c^{2}}{e}
$$

Equation 3.6 expresses the electrode voltage in terms of the plate separation, the radius of curvature of the trajectory, and the Lorentz energy factor of the beam. The spin rotator built for the GTL has the same design as the one used at CEBAF and manufactured by the $\mathrm{U}$. of Illinois.[38][39] The plate separation is $d=1.695 \mathrm{~cm}$ and the radius of curvature on the plane of deflection is $r_{0}=11.081 \mathrm{~cm}$. Substituting these values into Eqn. 3.6 gives an expression for the electrode voltage as a function of the beam energy

$$
V=\frac{\gamma^{2}-1}{\gamma}(39.0824 \mathrm{kV})
$$

Utilizing the theory of relativity, the Lorentz energy factor can be expressed in terms of the beam energy as

$$
\gamma=1+\frac{T}{m c^{2}}
$$

where $T$ is the beam kinetic energy. Table 3.1 utilizes Eqns. 3.1, 3.7 and 3.8 to summarize the angle of trajectory deflection and electrode voltage required to produce beams with transverse spin orientation.

The spin rotator at CEBAF was designed for a $100 \mathrm{keV}$ beam. Its angle of trajectory deflection is $107.7^{\circ}$. In addition, the electrodes have a toroidal shape (see Fig. 3.7) that form a radially inhomogeneous electrostatic sector field.[37][38] Such field provides additional focusing in the y direction. Since the typical beams used in the GTL have an energy of $120 \mathrm{keV}$, the CEBAF spin rotator will produce spin precession angles which are less than $90^{\circ}$. The spin precession angle for a $120 \mathrm{keV}$ beam is obtained by substituting $\gamma=1.23483$ and $\Theta_{\text {bend }}=107.7^{\circ}$ into Eqn. 3.1 


\begin{tabular}{|l|l|l|l|}
\hline$T(\mathrm{keV})$ & $\gamma$ & $\Theta_{\text {bend }}$ & $V(\mathrm{kV})$ \\
\hline 60 & 1.11742 & $100.5968^{\circ}$ & 8.6956 \\
\hline 80 & 1.15656 & $104.1312^{\circ}$ & 11.4092 \\
\hline 100 & 1.19570 & $107.6667^{\circ}$ & 14.0450 \\
\hline 120 & 1.23483 & $111.2024^{\circ}$ & 16.6101 \\
\hline 150 & 1.29354 & $116.5096^{\circ}$ & 20.3411 \\
\hline 180 & 1.35225 & $121.8196^{\circ}$ & 23.9474 \\
\hline
\end{tabular}

Table 3.1: Angle of trajectory deflection and electrode voltage required to produce beams with transverse spin orientation.

$$
\Theta_{\text {prec }}=87.1654^{\circ}
$$

Equation 3.9 will be used in section 3.3.3 to derive more precisely the polarization of beams measured with the GTL Mott polarimeter.

The GTL spin rotator was constructed with very high precision such that it can also be used as a spectrometer. For instance, the beam energy can be computed from the electrode voltage necessary to align the beam with the optic axis. This technique is
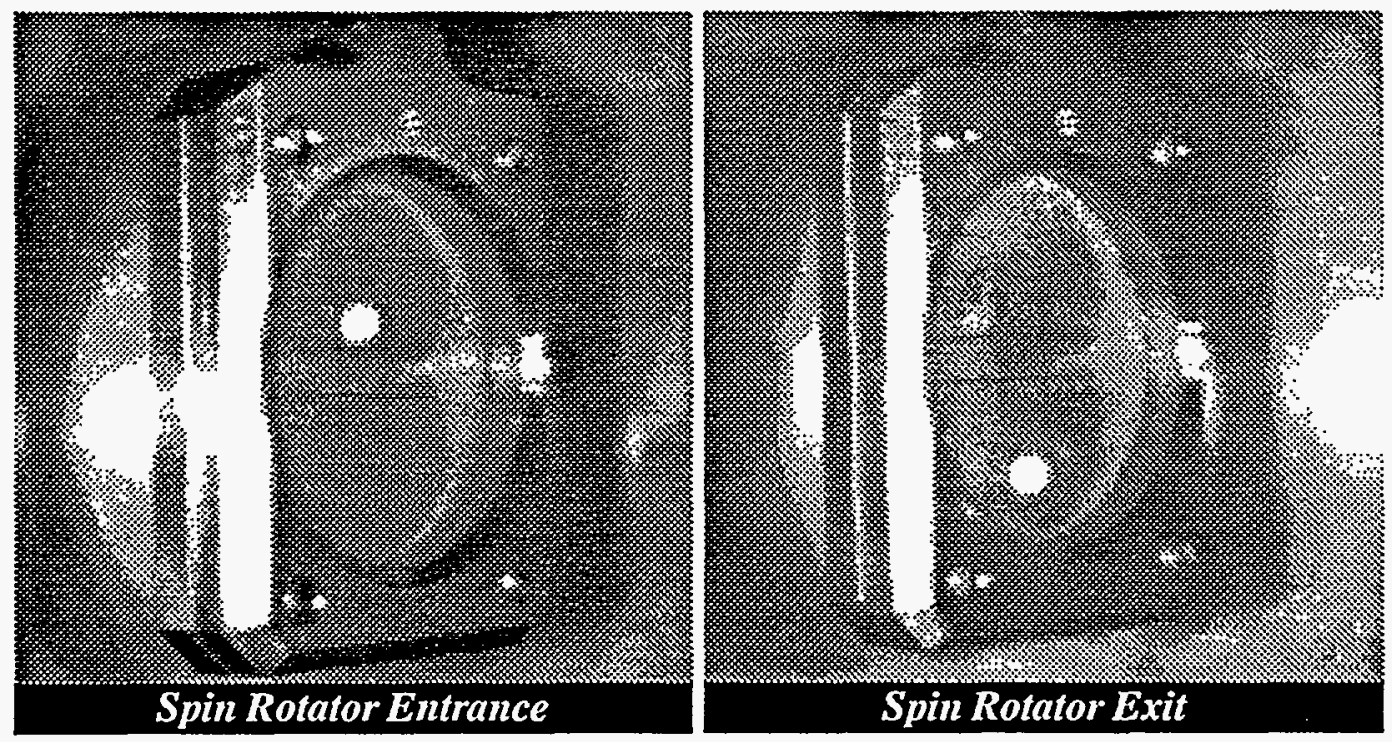

Figure 3.9: Alignment of the beam spots to the optic axis of the spin rotator. The beam energy is obtained from the voltage required to align the beam horizontally ( $x$ direction in Fig. 3.7 ) to the optic axis. 
illustrated in Fig. 3.9. On one occasion alignment of the spin rotator suggested that the beam energy was $10 \mathrm{keV}$ higher than that indicated by the gun power supply. A second independently calibrated gun power supply agreed with the extra $10 \mathrm{keV}$ observation.

\subsubsection{Mott Polarimeter}

The Mott polarimeter is the newest beam diagnostic tool in the GTL. It can be used to study the polarization of new photocathodes in the same environment that they experience in the accelerator. More importantly, however, the polarimeter can be used to study the effect of $\mathrm{CL}$ on polarization. It is conceivable that as the photoemission properties of the photocathode change at high current densities, the beam polarization could also be affected. The GTL is the only laboratory that can simultaneously study the beam production and polarization of photocathodes used in linear accelerators. This section will review the basic physics of Mott scattering and it will discuss some of the design features of the GTL polarimeter.

The theory of operation of Mott polarimeters is based on the spin-orbit interaction between the beam electrons and the target nuclei. The Hamiltonian of the spin-orbit interaction is given by

$$
H_{\text {spin-ordis }}=\frac{Z e^{2}}{2 m^{2} c^{2} r^{3}} \mathbf{L} \cdot \mathbf{S}
$$

where $Z$ is the atomic number of the nucleus, $e$ and $m$ are the charge and mass of the electron, respectively, $c$ is the speed of light, $r$ is the separation between the electron and the nucleus, $\mathbf{L}$ is the orbital angular momentum operator, and $\mathbf{S}$ is the spin angular momentum operator.[40][1] The scattering cross-section of the spin-orbit interaction is given by

$$
\sigma(\theta)=I(\theta)[1+S(\theta) \mathbf{P} \cdot \mathbf{n}]
$$


where $I(\theta)$ is the intensity of scattered electrons in the azimuthal angle $\theta, S(\theta)$ is the Sherman or asymmetry function of the target, $\mathbf{P}$ is the beam polarization, and $\mathbf{n}$ is the normal to the scattering plane. For a gold target $(Z=79)$ and beam energy of $100 \mathrm{kV}$, the Sherman function is largest at $\theta=120^{\circ}$. Note that the term $S(\theta) \mathbf{P} \cdot \mathbf{n}$ is largest when the polarization vector is perpendicular to the scattering plane. By flipping the polarization sign in Eqn. 3.11, a large change in the scattering cross section occurs. It will be shawn later that the beam polarization can be obtained from this scattering asymmetry.

Figure 3.10 illustrates how the beam polarization is measured in Mott polarimeters. Two detectors are placed at the azimuthal angle where the scattering cross section is largest. The scattering plane is thus defined by the incident beam and these detectors. The beam polarization is transformed perpendicular to the scattering plane in order to maximize the scattering cross section. A beam with positive helicity polarization will have its polarization vector pointing into the scattering plane (clear spin vector in Fig. 3.10). A beam with negative helicity polarization will have its polarization vector pointing out the scattering plane (dark spin vector in Fig. 3.10). From Eqn. 3.11, the positive helicity electron will have a preference to scatter to the lower detector while the negative helicity electron will have a preference to scatter to the upper detector. The beam polarization is computed by measuring the asymmetry in the signals of these detectors when the helicity is switched.

As mentioned above, the beam polarization can be computed from the asymmetry measured in the detector signals. Let the top detector be $T$ and the bottom detector be $B$. The expected number of counts in detector $B$ for positive helicity electrons is given by

$$
B_{\uparrow}=C_{B}(1+P S)
$$

where $C_{B}$ is a proportionality constant, $P$ is the beam polarization and $S$ is the Sherman function of the target. For negative helicity electrons, the expected number of counts is given by

$$
B_{\downarrow}=C_{B}(1-P S)
$$




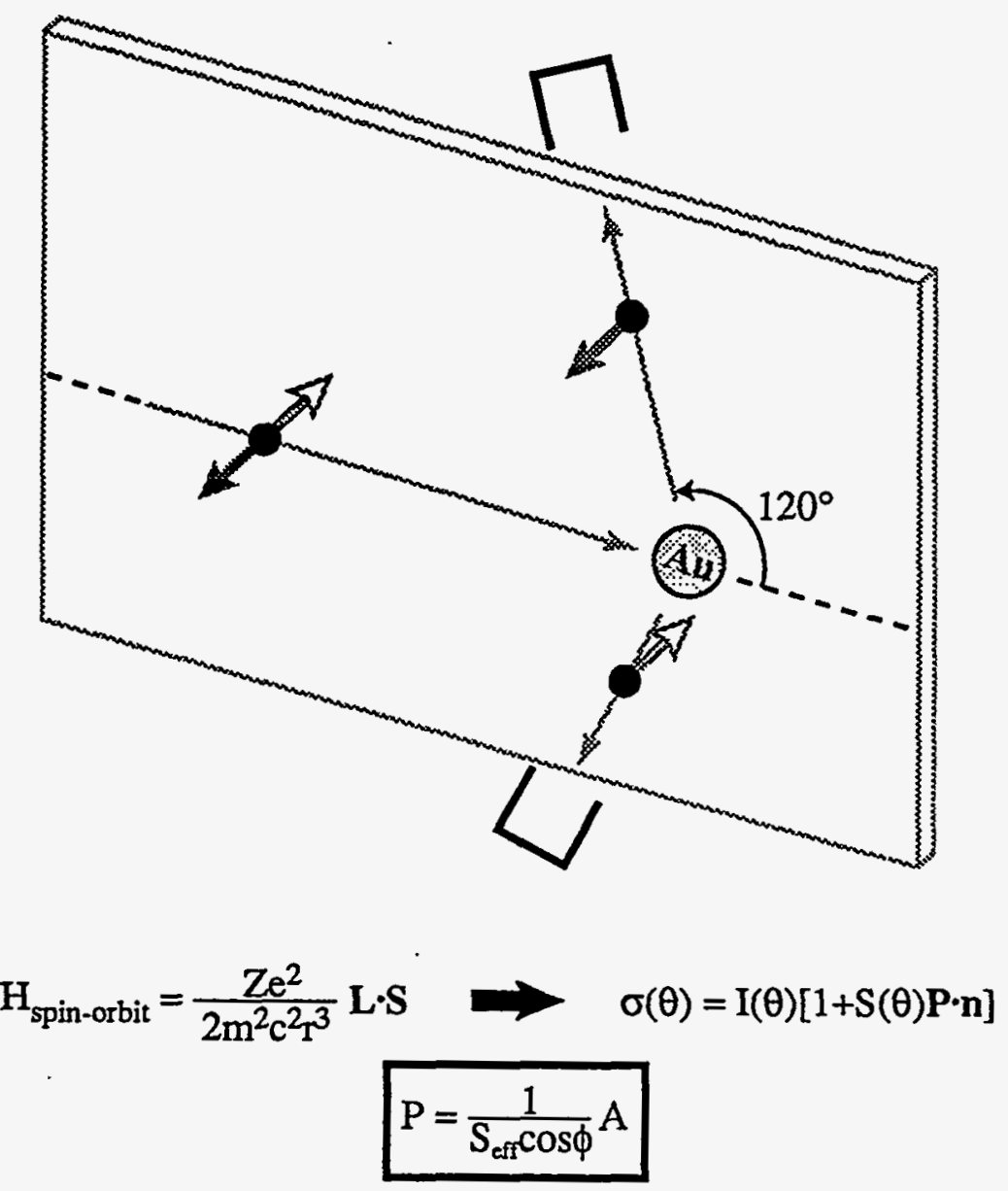

Figure 3.10: Measurement of an electron beam polarization with Mott scattering. The spin-orbit interaction between the beam electrons and the target nuclei causes an symmetry in the azimuthal scattering. Positive helicity electrons (clear arrows) will tend to scatter more favorably to the bottom detector while negative helicity electrons (dark arrows) will tend to scatter more favorably to the upper detector. By measuring the asymmetry in the signals of these detectors, the beam polarization can be computed.

Dividing Eqn. 3.12 by 3.13 results in

$$
\frac{B_{\uparrow}}{B_{\downarrow}}=\frac{1+P S}{1-P S}
$$

Note that the polarization can already be obtained from Eqn. 3.14 by solving for $P$. However, detector misalignments can result in erroneous values for $S$. These misalignment errors can be averaged out by also utilizing the top detector in the 
computation of the polarization.[40][41] Repeating the same steps given in Eqns. 3.12, 3.13 , and 3.14 for the top detector results in

$$
\frac{T_{\downarrow}}{T_{\uparrow}}=\frac{1+P S}{1-P S}
$$

The beam polarization can be simply computed by multiplying Eqns. 3.14 and 3.15 and solving for $P$

$$
P=\frac{1}{S} A
$$

where $A$ is defined as the scattering asymmetry and given by

$$
A=\frac{\Delta-1}{\Delta+1}, \Delta=\sqrt{\frac{T_{\uparrow} B_{\downarrow}}{T_{\downarrow} B_{\uparrow}}}
$$

Equations 3.16 and 3.17 are the standard equations used to compute the beam polarization utilizing Mott polarimeters. In deriving Eqn. 3.16 it was assumed that the beam polarization was transverse to the scattering plane, i.e. $\mathbf{P} \cdot \mathbf{n}=P$. However, it was shown in section 3.3.2 that the angle of precession of a $120 \mathrm{keV}$ beam traveling through the CEBAF spin rotator was less than $90^{\circ}$ and given by Eqn. 3.9. Thus Eqn. 3.16 can be corrected easily by reintroducing a cosine factor

$$
P=\frac{1}{S \cos \phi} A
$$

where $\phi=2.83462^{\circ}$ for a $120 \mathrm{keV}$ beam. It will be shown in section 4.1 that this correction is rather small compared to the systematic errors of the instrument.

One key problem with Eqn. 3.18 is that it assumes knowledge of the Sherman function. The Sherman function is very sensitive to the composition and thickness of the target and thus it must be measured carefully for each Mott polarimeter. In the scattering of 
electrons off a solid target there are two types of deflections which differ from the ideal single atom scattering: plural scattering in which the electron undergoes large-angle collisions and multiple scattering corresponding to many small-angle scattering events. These deflections have the effect of reducing the scattering asymmetry. Thus to properly calibrate a Mott polarimeter it is necessary to find the effective Sherman function that will yield the true beam polarization for the measured asymmetry.

The most common method used to measure the effective Sherman function $S_{\text {eff }}$ in Mott polarimeters is known as foil thickness extrapolation. In this method the scattering asymmetry is measured as a function of the (target) foil thickness. As the foils become thinner, the scattering asymmetry will approach that of the ideal single atom. This method assumes a linear dependence between scattering asymmetry and target thickness. By fitting the data points to a straight line and by using the theoretical value of the Sherman function at zero thickness, the beam polarization can be measured. The effective Sherman function for a given foil is then given by the measured asymmetry divided by the beam polarization

$$
S_{e f f}=\frac{A_{\text {meas }}}{P \cos \phi}
$$

Figure 3.11 illustrates the target holder in the GTL Mott polarimeter. The image on the left
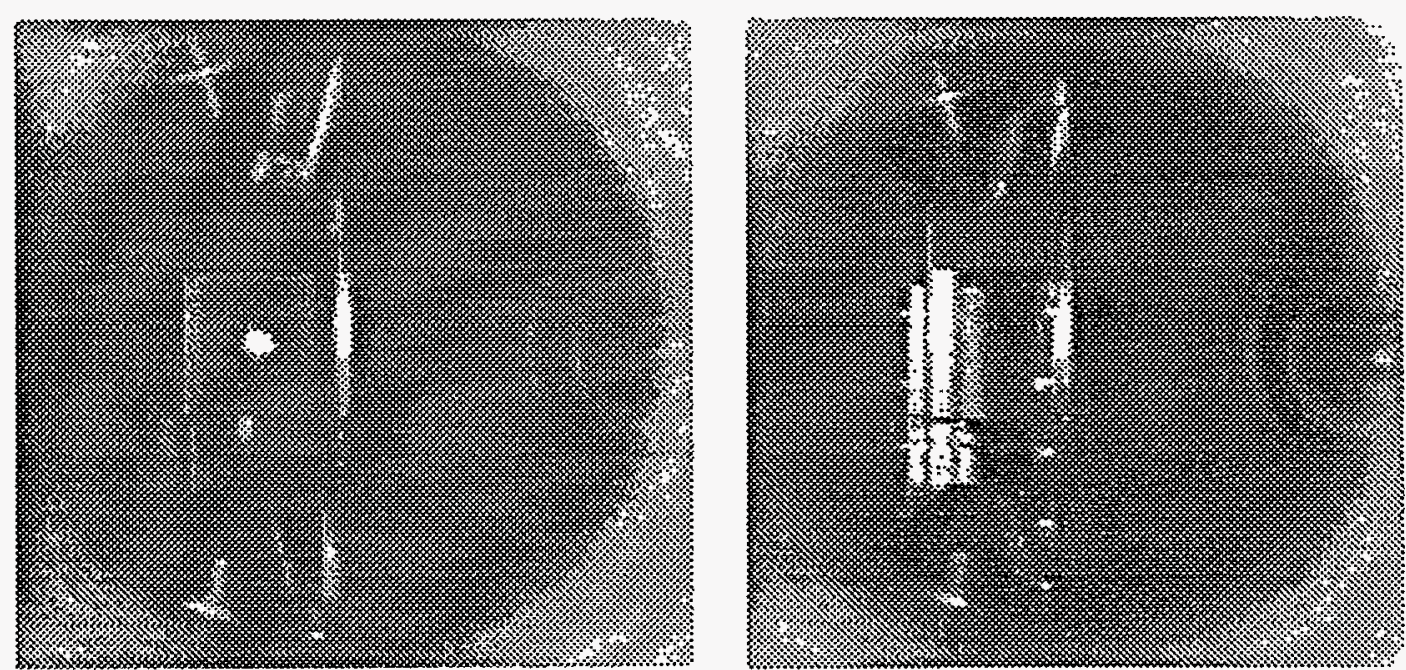

Figure 3.11: Target holder in the GTL Mott polarimeter. The image on the left shows the fluorescent alignment piece while the image on the right shows the first of four foil targets. 
in Fig. 3.11 shows the fluorescent aluminum oxide piece which is used to align the beam to the axis of the detectors. The image on the right in Fig. 3.11 shows the first of four foil targets. These four targets include: $700 \AA$ gold on $1.3 \mu \mathrm{m}$ carbon, $400 \AA$ gold on $1.3 \mu \mathrm{m}$ carbon, $200 \AA$ gold on $1.3 \mu \mathrm{m}$ carbon, and $1.3 \mu \mathrm{m}$ carbon.

The foil thickness extrapolation method has been widely criticized as an inaccurate calibration procedure for Mott polarimeters. This criticism holds that many of this method's underlying assumptions are false. For instance, as the targets become thicker, less electrons scattered deep in the target will reach the detectors. This effect will tend to flatten out the dependence of scattering asymmetry versus target thickness. In addition, the electrons scattered off the carbon backing will reduce the asymmetry measured at the detectors. Even if the electrons scattered from the carbon are subtracted from the detector signal, the signal to noise ratio for the thin foils is so small that it will increase the uncertainty in the asymmetry measurement. Many of the problems with foil thickness extrapolation can be reduced both by using only very thin foils and by using energy discrimination (thus eliminating the inelastic scattering in the detector signal).

Because the foil thickness extrapolation method presents so many problems, the Sherman function for the GTL Mott polarimeter was measured in a different way. A 'standard' source of polarized electrons was instead developed to determine the effective Sherman function.[42] The polarization of the standard source was measured at various institutions which had developed highly accurate, self-calibrating polarimeters. The polarization value for the standard source used in the calibration of the GTL polarimeter was the average of the polarization values at those institutions. The calibration procedure for the GTL polarimeter is explained in more detail in section 4.1. One additional advantage of using a standard polarization source is that it helps to compare the polarimeter calibrations of the various institutions. For instance, some institutions that were reporting high values for its polarization measurements were found to have a higher than average polarization value for the standard source.

The standard source of polarized electrons had to be simple and its properties had to be reproducible in every measurement in order not to introduce errors into the calibration procedure of the GTL polarimeter. The standard photocathode profile is shown in Fig. 3.12. The arsenic passivation layer was used to protect the photocathode surface from the time it was grown to the time it was first activated. It is believed that this layer faded from 


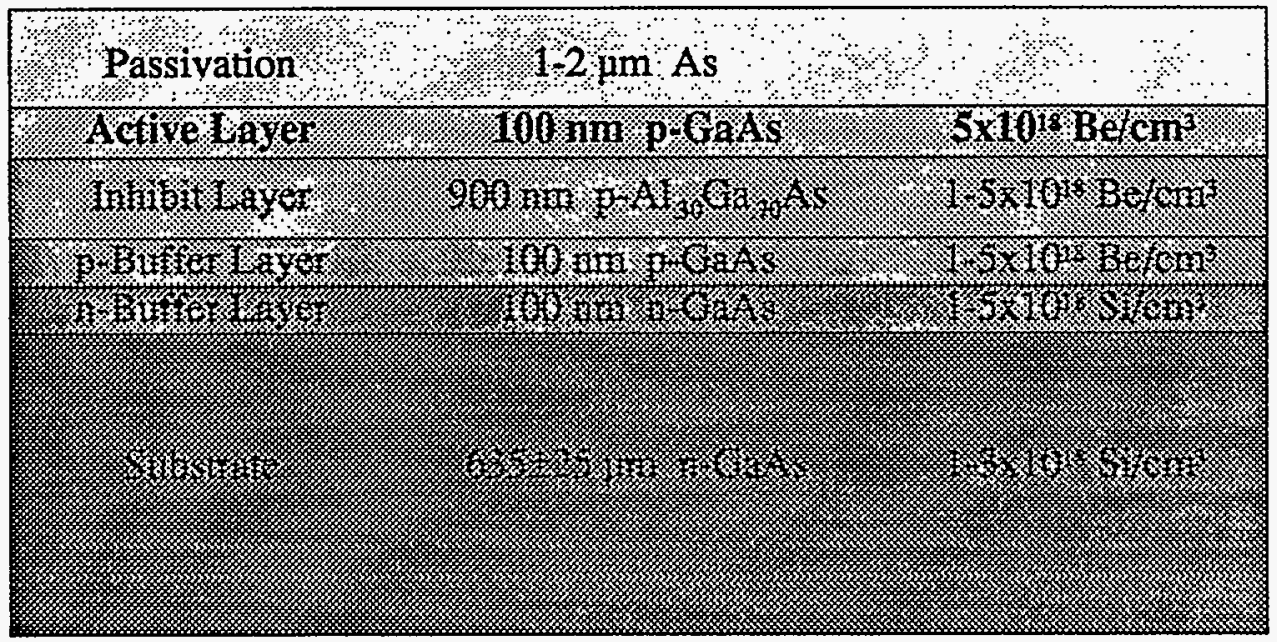

Figure 3.12: Profile of the standard GaAs photocathode. The thickness of the layers is not to scale.

the surface rather quickly. The active layer (i.e. the source of polarized electrons) was made of $100 \mathrm{~nm}$ of MBE grown GaAs with a beryllium impurity concentration (p-doping) of $5 \times 10^{18}$ atoms $/ \mathrm{cm}^{3}$. The inhibit layer was made of $\mathrm{p}-\mathrm{Al}_{.30} \mathrm{Ga}_{.70} \mathrm{As}$. The bandgap of the inhibit layer is larger than the bandgap of $\mathrm{GaAs}$ in order to ensure that it will not contribute any electrons to the photoemission process and that it will block any electrons exited in the buffer layers from reaching the photocathode surface. In addition, the inhibit layer has the same crystal lattice constant and doping concentration as the active layer in order to prevent any strain between the two layers. The buffer layers act as a transition between the substrate and inhibit layers. The substrate layer is the supporting structure of the photocathode. It is thick enough to prevent bending and other structural deformations in the photocathode. 


\section{Chapter 4}

\section{Experimental Results}

After all the modeling on the behavior of high-polarization photocathodes is complete, experimental testing must ultimately be performed to discern which ideas are valid. During this testing period it is possible to discover physical behavior that was not predicted by any model and that can surprise the scientists. The GTL is equipped with instruments that can test the charge production and polarization characteristics of a photocathode. Some of the instruments used in these tests include: a beam current measuring device (nanoamp meter), a GAPM and a FFC to study the temporal profile of the beam, a photoluminescent screen to observe the cross-sectional profile of the beam, a Mott polarimeter to measure the average beam polarization, and Ti-Sapphire and diode lasers to probe the photoemissive properties

of the semiconductor. In addition, the laboratory has capabilities to lower the temperature of the photocathode to $150^{\circ} \mathrm{K}$.

Of the above-mentioned instruments, the Mott polarimeter beamline was the latest addition to the laboratory and had to be commissioned for operation. A number of systematic tests were performed on the polarimeter to study the reproducibility of the polarization measurements and to find any polarization dependence on the beam transport through the beamline. Once the polarization measurements proved to be consistent and reproducible, the study of the fundamental photocathode behavior began. These studies included the polarization dependence on $\mathrm{QE}, \mathrm{Cs}$ deposition, temperature, laser intensity, bunch to bunch effects, and CL.

A few of the measurements on the photocathode proved to be more difficult to make than originally thought. For instance, the test of polarization dependence on laser intensity involved the use of very small beams. These beams were easy to transport from the gun to the Mott target but their current was difficult to measure because the nanoamp meter had limited sensitivity and because the gun dark current was large compared to the photoemitted current. The biggest drawback to the experimental program, however, might have been the 
lack of a tunable-wavelength laser. Such laser is necessary to study the vacuum level at the semiconductor surface, to measure the change of the semiconductor bandgap with temperature, and to study more carefully the beam polarization dependence on photon energy.

The calibration of the Mott polarimeter was a very important step in the experimental program. Many polarization measurements under various beam conditions were performed to study the reproducibility and reliability of the results. In addition, the standard source of polarized electrons was used to obtain the effective Sherman function of the GTL polarimeter. The effective Sherman function determines the absolute calibration of the instrument.

One of the first tests on the inner workings of the photocathode itself was the study of how the beam polarization varies with the surface vacuum level. This study was known as the polarization dependence on $\mathrm{QE}$ test. The observation that polarization is higher at higher vacuum levels (low $Q E$ ) was reconfirmed.[27] A substantial drop in polarization with new cesium deposition on the photocathode surface was also reconfirmed. However, careful analysis of the polarization dependence on $\mathrm{QE}$ suggests that two different effects might be at work. The first is that polarization drops when electrons scatter with the recently deposited Cs layer and the other effect is that low polarization electrons are filtered out by a higher vacuum level.

Since many spin relaxation models have a strong temperature dependence, a lot of motivation existed to test how the polarization was affected by the photocathode temperature. It was found in our tests, however, that polarization had a weak dependence on temperature. It seems that the short active region in the SLAC photocathodes limits the time that the electrons spend inside the material and thus reduces the effect of temperaturedependent spin relaxation mechanisms.

The polarization behavior under CL operation proved to be the most interesting effect. As it had been observed at the SLAC injector, no appreciable change in polarization was observed between low and high laser intensities for full photocathode illumination. However, for small and intense laser spots, a significant drop in polarization was observed. This was the first time this observation had been obtained at SLAC. This curious result might shed more information about the behavior of electrons near the surface of the semiconductors. In regards to the CL effect itself, careful analysis of the charge 
versus illumination intensity curve suggests that, for a given $\mathrm{QE}$, there might be an intrinsic semiconductor limit to the current density that can be extracted from these photocathodes.

One experiment that did not work as expected was the study of how an intense electron bunch affects the polarization of a second bunch. It had been observed that if a first bunch drives the photocathode into $\mathrm{CL}$ then the charge of a second bunch will be reduced.[3] The main difficulties with this test included: nonreproducibility of the spatial superposition of the first and second laser pulses due to the spatial jitter of the laser, high instability of the laser intensity, and drift of the laser intensity as the laser pulse timing was changed.

An interesting experiment that would complement the studies performed in the GTL would be a study of the polarization and QE dependence on the photocathode crystal orientation. Several papers indicate that the electron behavior at the semiconductor surface and in the bulk differs for various crystal orientations.[10][43][44][45] Completion of this experiment will yield important information for the future development of high polarization and high $\mathrm{QE} \mathrm{GaAs}$ photocathodes.

The experimental program for this dissertation was a mixture of many interesting and important results together with a few disappointing results. An important realization from the experimental program is that improvements in the laboratory instrumentation will aid the ability to perform new experiments on these photocathodes. Section 5 will discuss how the results of this experimental program together with future experiments will help to improve the charge production and polarization of $\mathrm{GaAs}$ photocathodes.

\subsection{Mott Polarimeter Calibration}

The new Mott polarimeter in the GTL was calibrated using the standard GaAs source of polarized electrons described in section 3.3.3. The calibration procedure was quite simple; it consisted of finding the effective Sherman function $S_{\text {eff }}$ that would yield the polarization of the standard source for the measured asymmetry. The relationship between polarization, measured asymmetry, and effective Sherman function is given again by 


$$
P=\frac{A_{\text {meas }}}{S_{e f f} \cos \phi}
$$

The standard GaAs photocathode was thoroughly tested in the Cathode Test Laboratory (CTL) and the reproducibility of its polarization was confirmed under various external conditions. The polarization of the standard source was also measured by very accurate, self-calibrating polarimeters at various institutions.[42] These measurements on the standard source can help scientists to compare the polarimeter calibrations being used at the various institutions. A graph depicting the typical plot of polarization and $Q E$ versus wavelength of illumination for the standard source is shown in Fig. 4.1. The measurements shown in Fig. 4.1 were performed by Greg Mulhollan in the CTL. After several measurements, a mean polarization value of $43.4 \%$ with $841 \mathrm{~nm}$ illumination was obtained. These measurements also showed virtually no polarization dependence on $\mathrm{QE}$ for $\mathrm{QE}$ values between $0.1-1.2 \%$ and $841 \mathrm{~nm}$ illumination. In addition, the polarization showed no dependence on wavelength, for wavelengths between $800-890 \mathrm{~nm}$. Three semiconductor wafers were grown with the same standard source methodology as described in section 3.3.3. Photocathode samples from all three wafers yielded the same

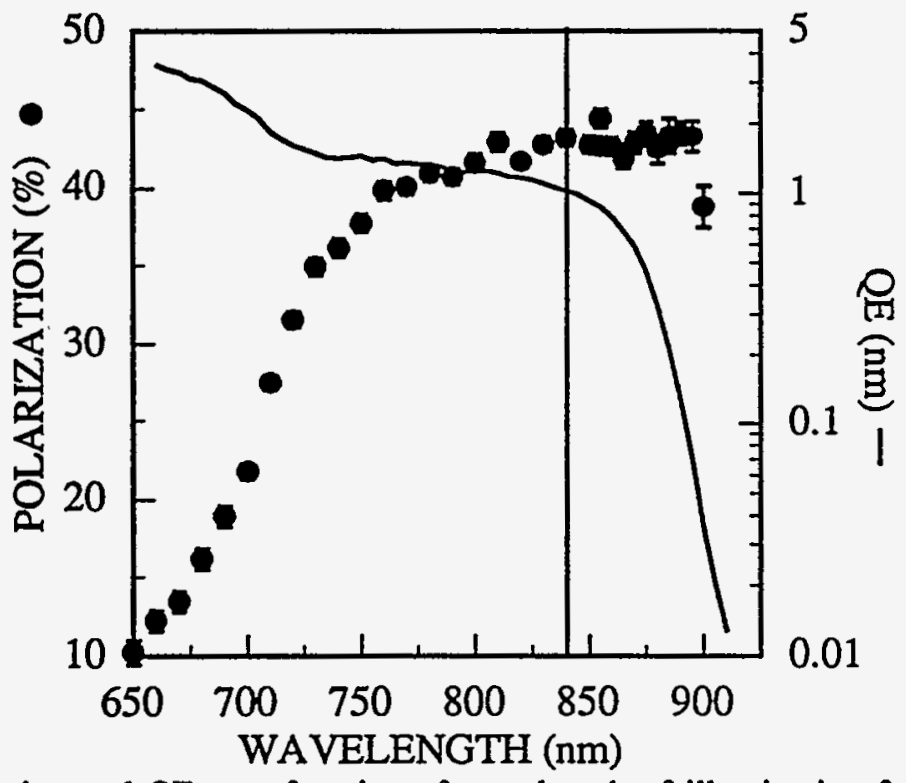

Figure 4.1: Polarization and $\mathrm{QE}$ as a function of wavelength of illumination for the standard GaAs photocathode. The vertical line at $841 \mathrm{~nm}$ represents the wavelength at which the polarization measurements were performed across the various institutions. 
polarization results.

Samples of the standard source were also measured at various institutions with highly accurate, self-calibrating polarimeters. The results of these measurements are summarized in Table 4.1. All the polarization values were taken with $841 \mathrm{~nm}$ illumination. The institution with the best performing polarimeter and with the lowest measurement error was the University of California at Irvine. It measured a polarization value of $44.66 \pm$ 0.94\%. Unfortunately, the polarimeters at the University of Nebraska, Rice University, and University of Mainz in Germany had a variety of problems during their polarization measurements. Thus their results are not being used for our calibration purposes until reliability in their measurements can be reestablished. Other polarimeters whose measurements of the standard source are interesting because they have produced several publications are the PEGGY polarimeter at SLAC and the Mott polarimeter at Nagoya University in Japan. Both instruments obtained polarization values that were higher than the values from $U$. C. Irvine.

The standard source was also used to study the reproducibility of the asymmetry measurements of the GTL Mott polarimeter. The systematic studies performed on the polarimeter included: how the scattering asymmetry depends on position of the beam on the target (the beam position was steered around the fixed target), how the scattering asymmetry depends on the target itself (the target was moved vertically while the beam position was being held fixed), and how the scattering asymmetry depends on the beam

\begin{tabular}{|l|l|l|l|}
\hline Institution & Polarization & Systematic Error & Statistical Error \\
\hline U. C. Irvine & $44.66 \%$ & $0.89 \%(2 \%$ rel. $)$ & $0.3 \%$ \\
\hline U. Nagoya & $46.29 \%$ & $3.24 \%(7 \%$ rel. $)$ & $0.33 \%$ \\
\hline Rice U. & $\leq 42 \%$ & $\sim 3-4 \%$ & \\
\hline U. of Mainz & pending & & \\
\hline U. Münster & pending & & \\
U. Nebraska & pending & & \\
\hline SLAC CTL & $43.4 \%$ & $1.3 \%(3 \%$ rel.) & $0.4 \%$ \\
\hline SLAC PEGGY & $49.3 \%$ & $2.5 \%(5 \%$ rel.) & $0.2 \%$ \\
\hline
\end{tabular}

Table 4.1: Polarization of the standard GaAs photocathode measured at the various academic institutions. 
steering along the injector transport line. These studies were later performed with a strained photocathode which has almost twice the asymmetry of the standard source and where any deviations in the asymmetry measurements would have been amplified. Details of these systematic tests can be found in the Polarized Gun \#3 Logbook for 10/12/94 8/24/94, pages 76-77 (utilizing the $100 \mathrm{~nm} \mathrm{GaAs}$ standard photocathode), and in the Polarized Gun \#3 Logbook starting on 8/24/94, pages 25 (utilizing a $200 \mathrm{~nm}$ strained GaAs photocathode) and pages 54-60 (utilizing a $100 \mathrm{~nm}$ strained GaAs photocathode). These logbooks can be found in SLAC Bldg. 006, Room 109.

The systematic studies showed that consistent and reproducible measurements of the scattering asymmetry were easily obtained with the GTL polarimeter. Figure 4.2 shows the electron beam on the alignment target at the spot which maximizes the counts on both the top and bottom detectors. The white rectangle in Fig. 4.2 indicates the region on the targets where the asymmetry measurements yield consistent values. The systematic error in the polarization due to steering the beam around the target was $0.47 \%$ (absolute). This number was computed by measuring the mean scattering asymmetry within the white rectangle area. As the beam is steered away from this area, the increase in background counts reduces the scattering asymmetry. In addition, if the laser spot was moved around the photocathode, the value of the scattering asymmetry could have changed. It will be shown in sections $4.3,4.4$, and 4.5 that the beam polarization can depend on the laser position on the photocathode. Areas on the photocathode with different QEs or with

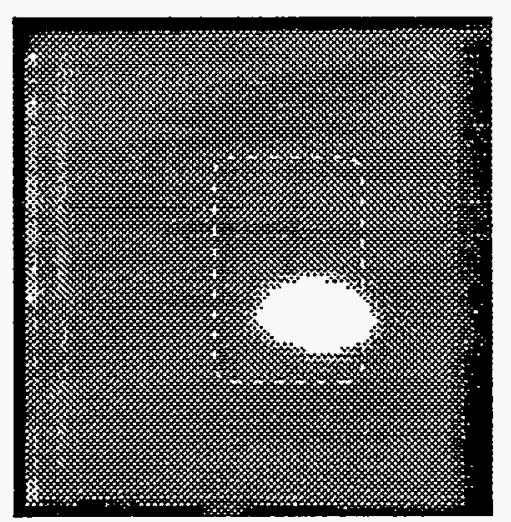

Figure 4.2: Electron beam impinging on the alignment target inside the Mott chamber. The location of the spot shown here had the highest count rates on both detectors. The white rectangle area represents the location were the asymmetry measurements were most consistent. The size of the alignment grid is $9 \mathrm{~mm}$ x $9 \mathrm{~mm}$. 
different degrees of charge saturation can yield different asymmetry values. One last important point to remember when making asymmetry measurements with the GTL polarimeter is that the gain of the photomultipliers can drift with time. For this reason, it is recommended that frequent pedestal measurements are taken during the course of an experiment.

A few instrumental problems had to be corrected during the commissioning of the GTL Mott polarimeter. Since these problems might occur again, it is worth mentioning them here in order to help future GTL users. The problems included: a bad Pockels cell yielding circular polarization values for the laser that were off by as much as $10 \%$, an improperly calibrated laser whose wavelength was off by as much as $20 \mathrm{~nm}$, an improperly calibrated gun HV power supply with a HV that was off by as much as 7\%, and a drop in polarization due to high-intensity laser illumination (see section 4.3). The circular polarization of the laser was improved and carefully measured with lower bound values between $99.3-99.7 \%$. A beam energy error of $7 \%$ produces a small error in the asymmetry of approximately $+0.1 \%$ due to a smaller spin precession $(a-0.1 \%$ effect on the asymmetry) and a higher (absolute value) Sherman function ( $a+0.2 \%$ effect on the asymmetry). The HV power supply was replaced with another one whose calibration agreed with the spin rotator (see section 3.3.2). Finally, to avoid lower asymmetry values due to non-linear effects from high laser intensities, only those measurements taken at low laser intensities were used for the Mott calibration.

For the purposes of calibrating the Mott polarimeter, the beam from the standard photocathode had to be of high quality. To reduce the statistical error during calibration, it was required to have all of the following: a good beam transport to the Mott target, a low beam position and intensity jitter, and a scattering signal-to-ADC noise ratio of 200 or greater in the Mott photomultipliers. The typical statistical error in the asymmetry measurements was much less than $1 \%$.

A total of 18 measurements were used to compute the effective Sherman function of the GTL Mott polarimeter. These measurements can be found in the Polarized Gun \#3 Logbook for 10/12/94 - 8/24/94, pages 111-114. The data points were taken using beams originating from various spots on the standard photocathode with $Q E$ values ranging between $0.06-1.4 \%$ and with the photocathode at room temperature. The mean asymmetry value for the standard photocathode computed according to Eqn. (3.17) was 
different degrees of charge saturation can yield different asymmetry values. One last important point to remember when making asymmetry measurements with the GTL polarimeter is that the gain of the photomultipliers can drift with time. For this reason, it is recommended that frequent pedestal measurements are taken during the course of an experiment.

A few instrumental problems had to be corrected during the commissioning of the GTL Mott polarimeter. Since these problems might occur again, it is worth mentioning them here in order to help future GTL users. The problems included: a bad Pockels cell yielding circular polarization values for the laser that were off by as much as $10 \%$, an improperly calibrated laser whose wavelength was off by as much as $20 \mathrm{~nm}$, an unproperly calibrated gun HV power supply with a HV that was off by as much as 7\%, and a drop in polarization due to high-intensity laser illumination (see section 4.3). The circular polarization of the laser was improved and carefully measured with lower bound values between $99.3-99.7 \%$. A beam energy error of $7 \%$ produces a small error in the asymmetry of approximately $+0.1 \%$ due to a smaller spin precession $(a-0.1 \%$ effect on the asymmetry) and a higher (absolute value) Sherman function ( $a+0.2 \%$ effect on the asymmetry). The HV power supply was replaced with another one whose calibration agreed with the spin rotator (see section 3.3.2). Finally, to avoid lower asymmetry values due to non-linear effects from high laser intensities, only those measurements taken at low laser intensities were used for the Mott calibration.

For the purposes of calibrating the Mott polarimeter, the beam from the standard photocathode had to be of high quality. To reduce the statistical error during calibration, it was required to have all of the following: a good beam transport to the Mott target, a low beam position and intensity jitter, and a scattering signal-to-ADC noise ratio of 200 or greater in the Mott photomultipliers. The typical statistical error in the asymmetry measurements was much less than $1 \%$.

A total of 18 measurements were used to compute the effective Sherman function of the GTL Mott polarimeter. These measurements can be found in the Polarized Gun \#3 Logbook for 10/12/94 -8/24/94, pages 111-114. The data points were taken using beams originating from various spots on the standard photocathode with $Q E$ values ranging between $0.06-1.4 \%$ and with the photocathode at room temperature. The mean asymmetry value for the standard photocathode computed according to Eqn. (3.17) was 


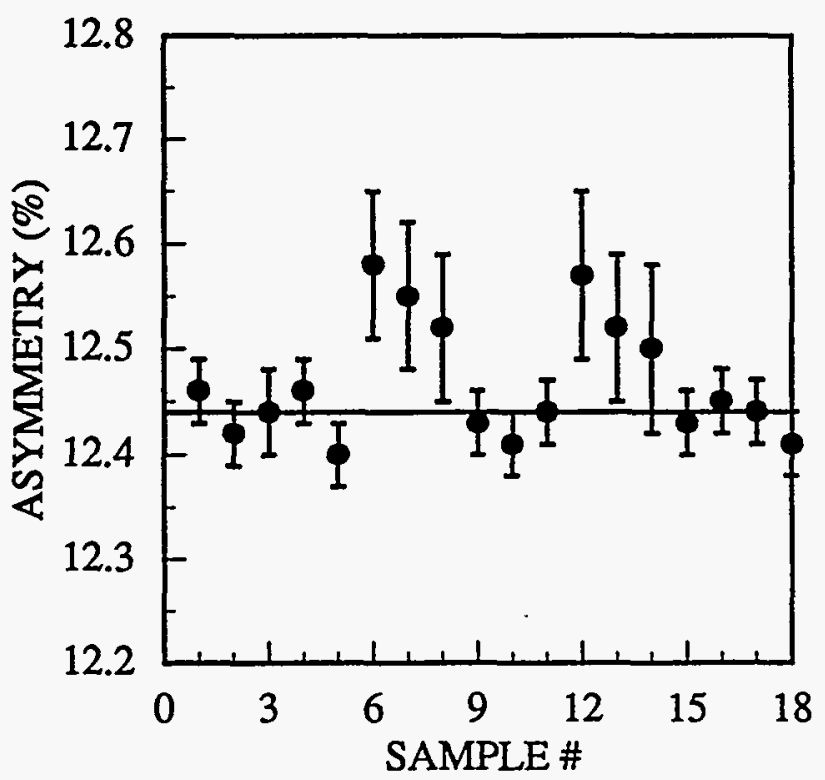

Figure 4.3: Calibration points taken from the standard GaAs photocathode. The horizontal line at Asy. = $12.44 \%$ represents the weighted average of the measurements. Note that the points with smaller errors have more weight on the average value of the asymmetry than the points with larger errors.

$$
A_{\text {meas }}=12.441 \pm 0.008 \%
$$

A plot of the data points used for the calibration can be seen in Fig. 4.3. The effective Sherman function for the GTL Mott polarimeter is obtained by rewriting Eqn. 4.1

$$
S_{e f f}=\frac{A_{\text {meas }}}{P \cos \phi}
$$

The polarization value $P$ that is most appropriate to use in Eqn. 4.3 is the one obtained at U. C. Irvine because it was, at the time of this dissertation, the only institution with an accurate, self-calibrating polarimeter that produced consistent results. The effective Sherman function for the GTL Mott polarimeter is obtained by substituting into Eqn. 4.3 the polarization value from $U$. C. Irvine in Table $4.1, P=44.66 \pm 0.94 \%$, and the standard photocathode asymmetry value in Eqn. 4.2

$$
S_{e f f} \cos \phi=0.2785 \pm 0.0059
$$


where the error is obtained from the propagation of errors of the asymmetry measurement in the GTL and the polarization measurement at $U$. C. Irvine. Thus, the relative error in the calibration constant in Eqn. 4.4 is on the order of 2.1\%. The calibration constant in Eqn. 4.4 was determined by utilizing a laser polarization of $99.0 \pm 0.5 \%$. If, however, the laser polarization used in future experiments is 99.9\%, then Eqn. 4.4 should be multiplied by a factor of 1.005 to compensate for the slightly higher asymmetry.

\subsection{Temperature Effects}

Temperature can have a significant effect on the basic properties of a semiconductor. The possibility that lower temperatures can improve the polarization of GaAs photocathodes is of special interest to SLAC. Many of the spin relaxation mechanisms discussed in section 2.3.2 have a strong temperature dependence. The spin relaxation dependence on temperature is supported by experimental evidence obtained using III-V semiconductor photocathodes.[14][44] Experiments performed in the GTL show only a small improvement in polarization at lower temperatures. Another interesting result from the GTL experiments is that the $\mathrm{QE}$ dependence on temperature for bulk photocathodes is the opposite to that of thin photocathodes. While the $\mathrm{QE}$ improves at lower temperatures for bulk photocathodes it gets worse for thin photocathodes. This result demonstrates that the photoemission process of bulk and thin samples can be quite different. This section will describe the experiments performed in the GTL that investigate how lower temperatures affect the photoemission and polarization characteristics of GaAs photocathodes.

The temperature of the photocathode in the SLAC polarized-electron guns is controlled by liquid nitrogen boil-off impinging on the back of the cathode support tube. This cooling mechanism is shown in Fig. 4.4. The temperature of the photocathode is taken to be the average of Gun_In and Gun_Out, the incoming and outgoing temperatures, respectively, of the nitrogen boil-off used to cool the photocathode puck. Even though the emitter tube and the puck are made of the excellent heat conductor molybdenum, there was concern that this average temperature might not the most accurate representation of the actual temperature of the photocathode. It is believed that the imperfect contact between the 


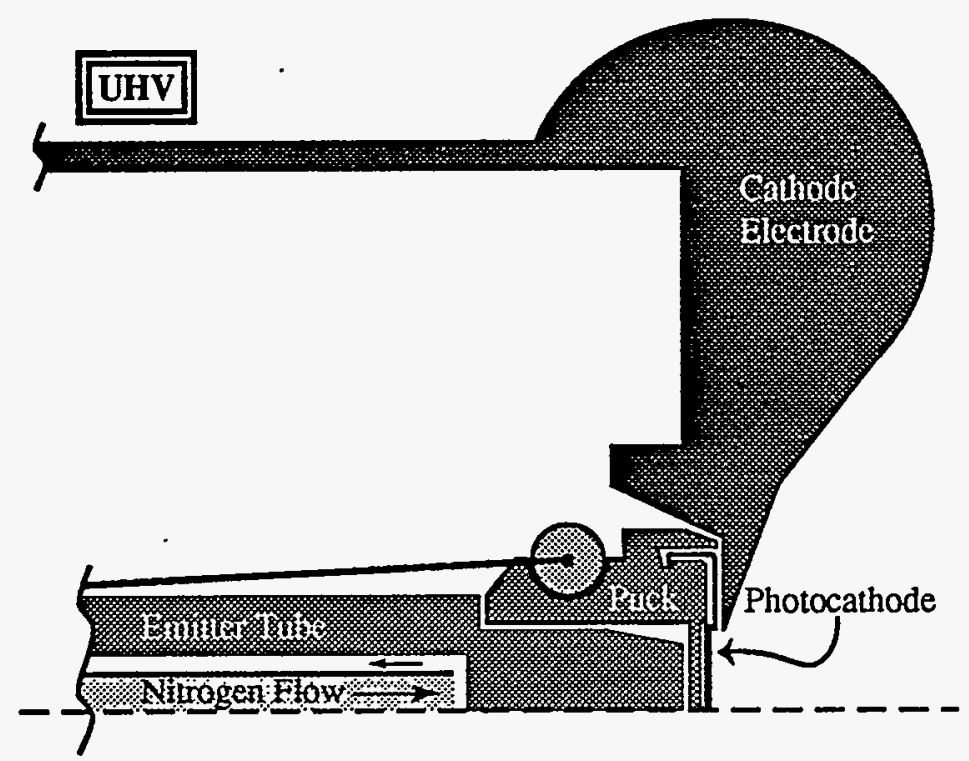

Figure 4.4: Cross-section of the polarized-electron gun electrode illustrating the cooling mechanism.

puck and the emitter tube and that the room-temperature thermal radiation can introduce a discrepancy between the actual temperature of the photocathode and the average value of Gun_In and Gun_Out. In addition, the temperature discrepancy between the photocathode and the in-and-out nitrogen seems to increase as the nitrogen temperature is changed very fast. This larger discrepancy in temperature between the in-and-out nitrogen and the photocathode is due to the poor heat conductivity of the photocathode (compared to the molybdenum) which prevents it from reaching equilibrium temperature very rapidly. Despite the assumed discrepancy in temperature between the photocathode and the in-andout nitrogen, it has been shown that the temperature at the end of the emitter tube does approximate the average temperature of the in-and-out cooling gas for slow enough changes in the gas temperature. This measurement was performed by Dave Schultz and can be found in the Polarized Gun \#3 Logbook of 10/12/92 through 8/24/94, pages 28-30. In this experiment, a thermocouple was placed against the molybdenum end of the emitter tube. The temperature of the emitter tube was compared to the average temperature of Gun_In and Gun_Out. A plot of these results is shown in Fig. 4.5. Even though the average temperature of 'Gun_In and Gun_Out is not a direct measurement of the photocathode temperature, Fig. 4.5 shows that it is a very good first order approximation to the temperature at the end of the emitter tube. 


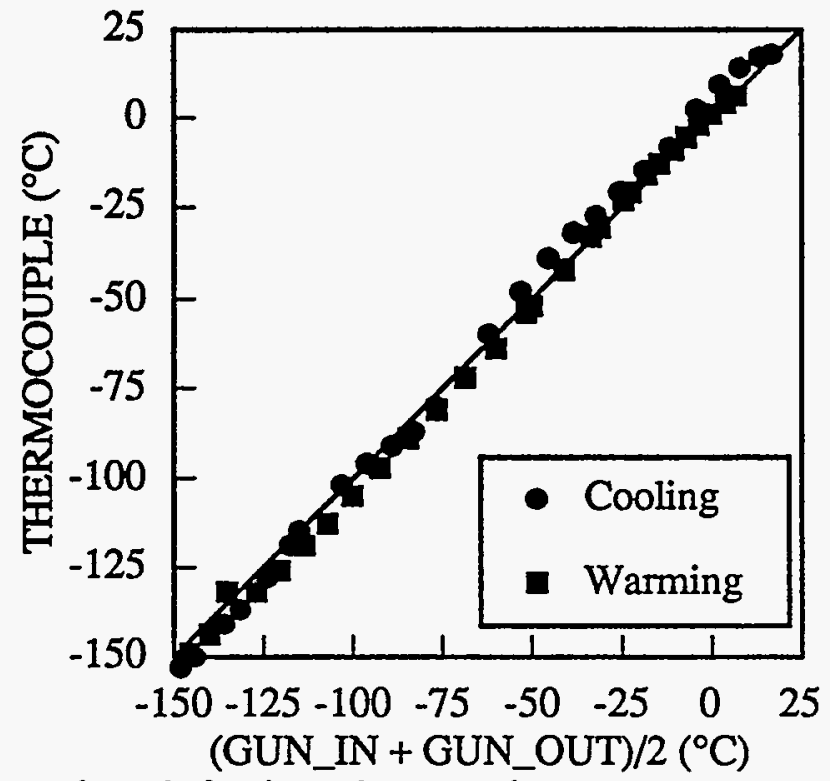

Figure 4.5: Temperature at the end of emitter tube versus the average temperature of the cooling nitrogen gas. The $45^{\circ}$ line represents a perfect correlation between the thermocouple temperature and the average temperature of the cooling gas.

A better method to measure relative changes in the photocathode temperature would be to monitor changes in the semiconductor bandgap (see, for example, section 2.1.2). The changes in the bandgap can be obtained by measuring either: the photoluminescence peak or the knee in the photoyield curve of the photocathode. To measure the photoyield curve all that is needed is a tunable-wavelength light source together with an ammeter to measure photocurrent. Unfortunately, the GTL was not equipped with a tunablewavelength light source at the time of this dissertation.

One of the first experiments performed involving the temperature of the photocathode was the study of the opposite $\mathrm{QE}$ dependence on temperature for bulk and thin GaAs. Figure 4.6 shows the $\mathrm{QE}$ at $750 \mathrm{~nm}$ illumination increasing with lower temperatures for a bulk GaAs photocathode. Note how a drop in temperature of $150^{\circ} \mathrm{C}$ causes an increase in $\mathrm{QE}$ of $50 \%$. The improvement of $\mathrm{QE}$ at low photocathode temperatures has been observed before.[2]

To understand the behavior of the $\mathrm{QE}$ at low temperatures, it is first necessary to take a careful look at the photoemission process. As discussed in section 2.2.1, the $\mathrm{QE}$ of 


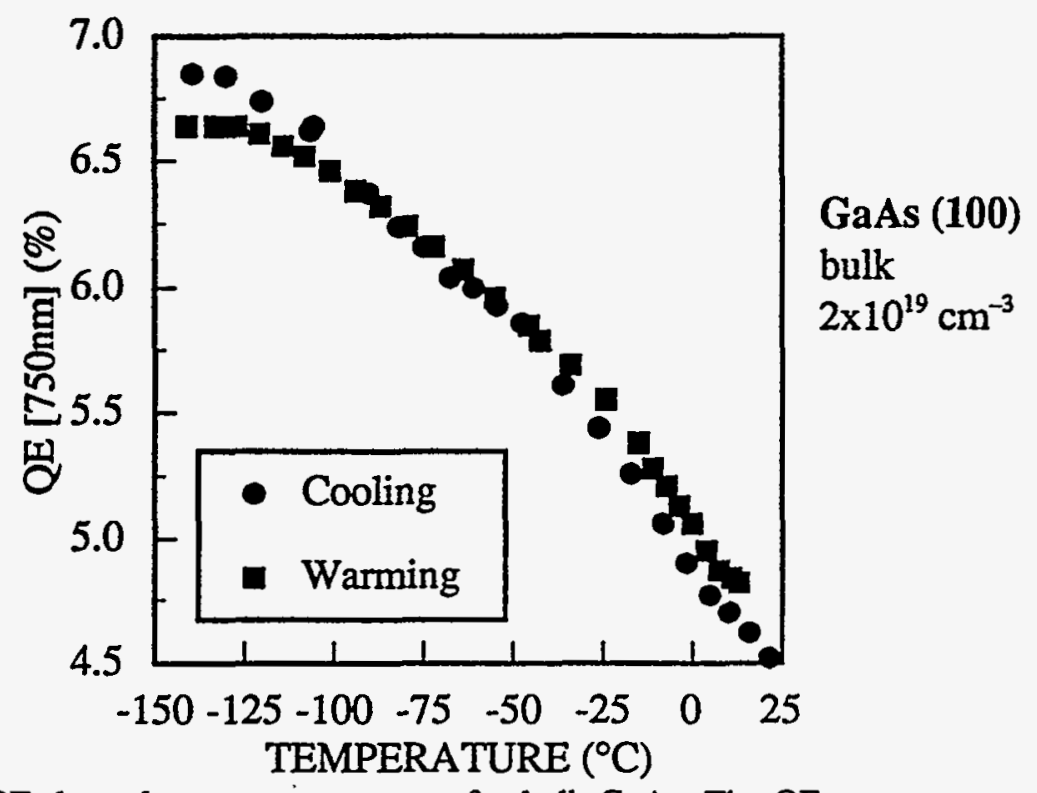

Figure 4.6: $Q E$ dependence on temperature for bulk GaAs. The QE measurements were taken for a wavelength of illumination of $750 \mathrm{~nm}$.

a semi-infinite photocathode can be derived utilizing Spicer's Three Step Model and is given by

$$
Q E=(1-R) \frac{\frac{\alpha_{e}}{\alpha} P_{e s c}}{1+\frac{l_{a}}{L}}
$$

Equation 4.5 has been shown to work very well for bulk photocathodes where the diffusion approximation is valid. All the variables in Eqn. 4.5 have some type of temperature dependence. However, the factors $(1-R)$ and $P_{\text {esc }}$ should have the same temperature dependence for both bulk and thin samples because these factors involve only the surface of the photocathode. The fraction of electrons excited above the vacuum level, $\alpha_{e} / \alpha$, decreases at lower temperatures due to a larger bandgap. On the other hand, the denominator, $1+l_{a} / L$, will decrease at lower temperatures due to an increase of the electron diffusion length in the conduction band.[8] The denominator in Eqn. 4.5 will be the dominating temperature-dependent factor in bulk semiconductors. The increase in the 
diffusion length improves photoemission because more electrons from deeper in the material are reaching the surface.

The $\mathrm{QE}$ behavior of thin photocathodes at low temperatures is the opposite of the behavior of bulk photocathodes, the $\mathrm{QE}$ decreases with lower temperatures. This effect is shown in Fig. 4.7. To properly describe the photoemission in thin photocathodes, Eqn. 4.5 must be modified for a photocathode of finite thickness.[7] To first order approximation, the $\mathrm{QE}$ is given by

$$
Q E=(1-R) \alpha_{e} P_{e s c} t
$$

where $t$ is the thickness of the photocathode. The larger semiconductor bandgap at low temperatures decreases both the absorption of light that excites electrons above the vacuum level $\alpha_{e}$ and the probability of escape $P_{e s c}$. Finally, note that the diffusion length $L$ was substituted in Eqn. 4.6 by the thickness of the photocathode $t$. This substitution is justified because the available source of electrons for photoemission is limited by the small thickness of the photocathode, not by the diffusion length. Thus the $\mathrm{QE}$ of thin photocathodes decreases at low temperatures because there are no more electrons available for

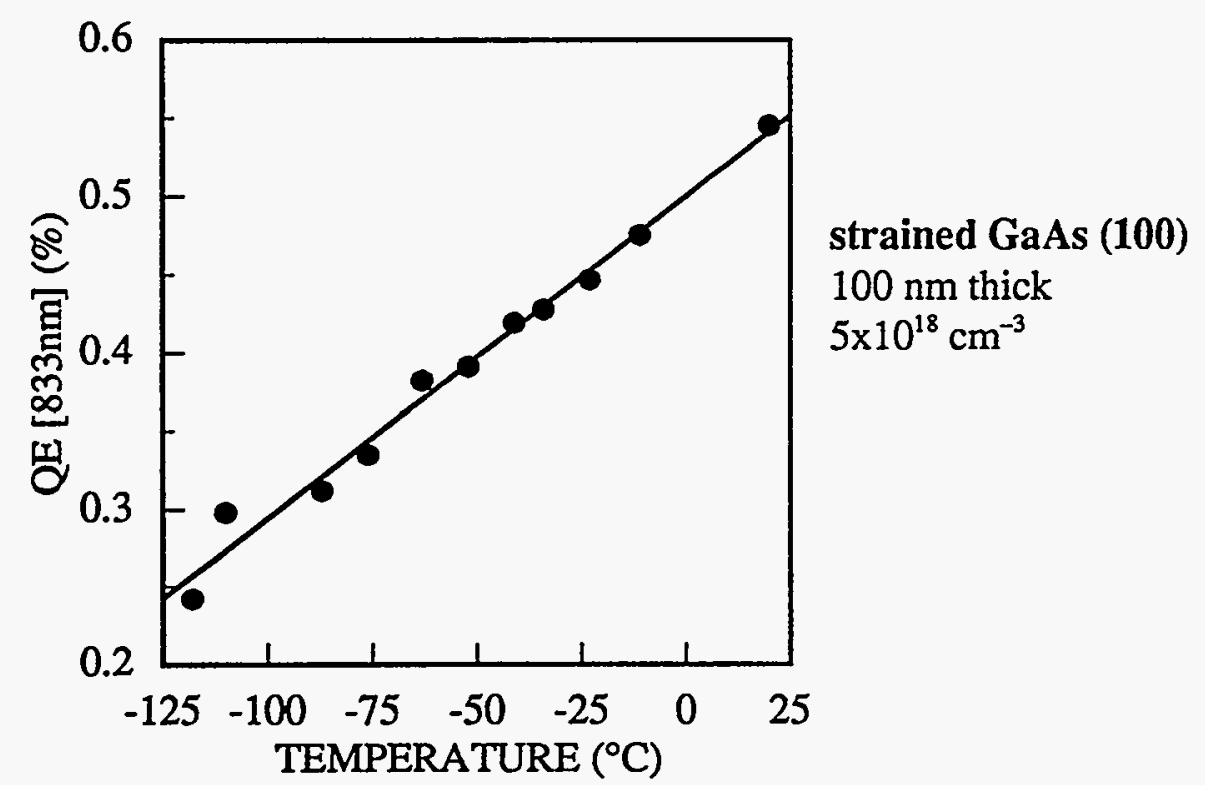

Figure 4.7: $\mathrm{QE}$ dependence on temperature for thin (strained) GaAs. The $\mathrm{QE}$ measurements were taken for a wavelength of illumination of $833 \mathrm{~nm}$. 
photoemission from deep in the material.

The studies involving the polarization dependence on temperature were probably the most exciting because they provided an opportunity to test the spin relaxation mechanisms. Many groups have already confirmed experimentally the polarization dependence on temperature of III-V semiconductor photocathodes.[5][44] Our tests in the GTL showed, however, that the polarization at lower temperatures improves very little for thin GaAs photocathodes. The polarization dependence on temperature for the $100 \mathrm{~nm}$ standard GaAs photocathode is shown in Fig. 4.8. For a temperature drop of over $150^{\circ} \mathrm{C}$, the relative improvement in polarization $(\Delta P / P)$ was only $\sim 1 \%$.

The polarization dependence on temperature for strained $\mathrm{GaAs}$ was more difficult to measure because the semiconductor has a narrow polarization peak versus wavelength near the bandgap. When the semiconductor is cooled, the bandgap increases and the peak in polarization shifts towards shorter wavelengths. For this reason, two laser cavities were set up; one tuned to a wavelength of $850 \mathrm{~nm}$ (the location of the polarization peak at room temperature) and the other one was tuned to a wavelength of $840 \mathrm{~nm}$. The polarization dependence on temperature for strained GaAs is shown in Fig. 4.9. Again, the relative improvement in polarization for a temperature drop of over $100^{\circ} \mathrm{C}$ was only $\sim 1 \%$. Note

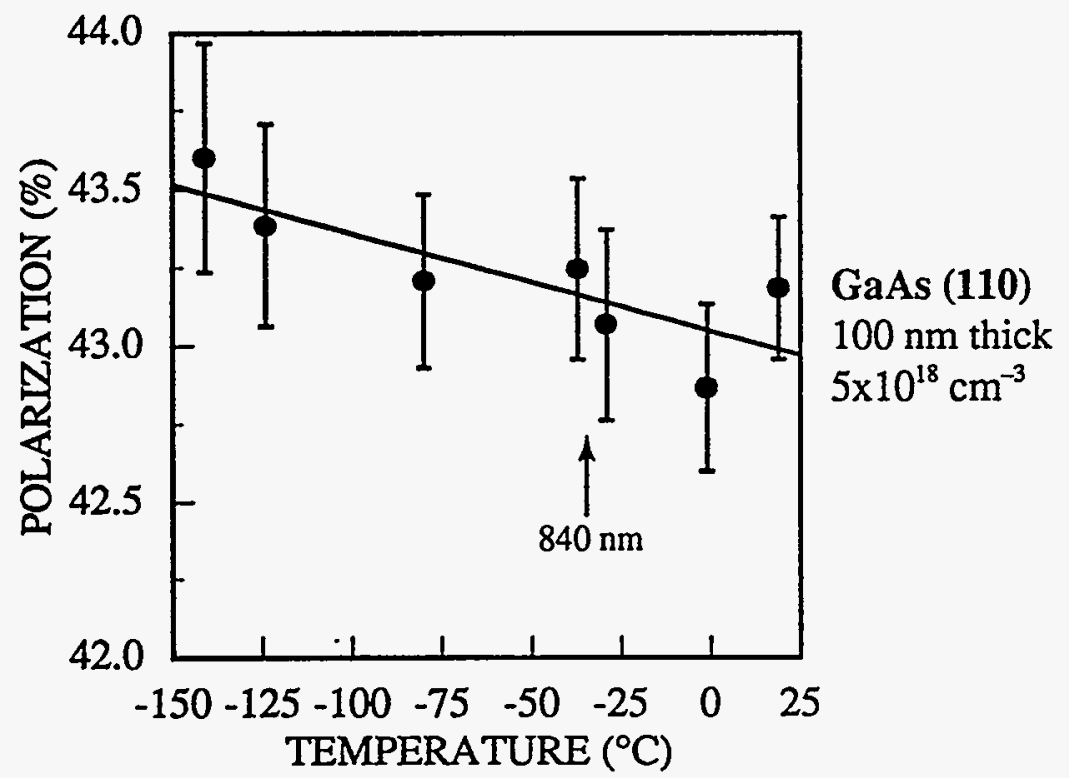

Figure 4.8: Polarization dependence on temperature for the $100 \mathrm{~nm}$ standard GaAs photocathode. The polarization measurements were taken at $840 \mathrm{~nm}$ illumination and at a $Q E[833 \mathrm{~nm}]$ of $0.90 \%$ at $25^{\circ} \mathrm{C}$. 


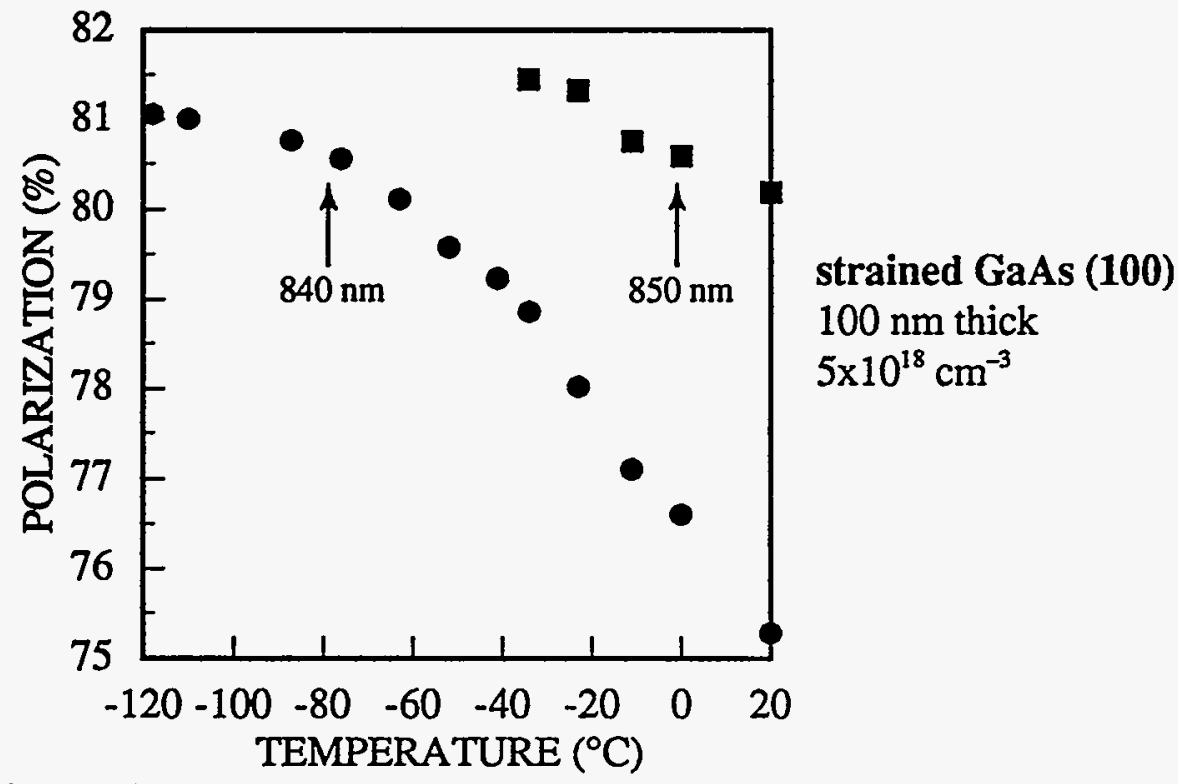

Figure 4.9: Polarization dependence on temperature for the $100 \mathrm{~nm}$ strained GaAs photocathode. The polarization measurements were taken at $840 \mathrm{~nm}$ and $850 \mathrm{~nm}$ illumination and at a $\mathrm{QE}[833 \mathrm{~nm}]$ of $0.55 \%$ at $20^{\circ} \mathrm{C}$.

that the peak in polarization for $840 \mathrm{~nm}$ illumination does not quite reach the peak in polarization for $850 \mathrm{~nm}$ illumination. This discrepancy might be due to the Pockels cell which was adjusted for $850 \mathrm{~nm}$ illumination. Discrepancies in the laser polarization for a $10 \mathrm{~nm}$ change in wavelength should be on the order of $1 \%$.

The measurements shown in Figs. 4.8 and 4.9 clearly indicate that the improvements in polarization at low temperatures are much smaller in thin photocathodes than the improvements reported for bulk photocathodes. Thin photocathodes were originally designed to minimize the time that electrons spend inside the semiconductor and thus to minimize the effect of the spin relaxation mechanisms. The results obtained in the GTL might be indicating that the photocathodes are already thin enough to minimize the effect of the spin relaxation mechanisms and that further reduction in temperature does not significantly improve polarization. In section 2.3 .2 , it was shown that the polarization of photoexcited electrons in the conduction band at the time of recombination was given by

$$
P=\frac{P_{0}}{1+\frac{\tau}{\tau_{s}}}
$$


The lifetime that an excited electron spends in a $100 \mathrm{~nm}$ semiconductor is very difficult to estimate because it depends strongly on the physical model used. An approximation of the lifetime of the electron can be obtained, however, by utilizing the thermalized velocity of an electron in the conduction band at room temperature $\left(4.0 \times 10^{7} \mathrm{~cm} / \mathrm{s}\right)$. [46] A ballistic electron (i.e. one that does not scatters) will travel $100 \mathrm{~nm}$ in $0.3 \times 10^{-12} \mathrm{sec}$. The real time of travel is expected to be slightly longer because the electron motion resembles a random walk more than ballistic motion. However, since the mean free path of electrons in $\mathrm{GaAs}$ is $l \approx 400 \AA$ (i.e. comparable to the distance of travel), the actual mean velocity is not expected to be much slower than the thermal equilibrium velocity.[46] From Fig. 2.11 in section 2.3.2, the spin relaxation time for highly doped GaAs is approximately $50 \times 10^{-12}$ sec. The polarization at room temperature for an electron in thin $\mathrm{GaAs}$ is obtained by substituting the spin relaxation time and the lifetime of a ballistic electron into Eqn. 4.7

$$
P \approx 0.994 P_{0}
$$

If the photocathode is cooled down sufficiently that the spin relaxation time becomes in essence infinite, the polarization in Eqn. 4.8 does not have much room to improve.

In summary, the time that a photoexcited electron spends inside the semiconductor has a significant effect upon the polarization during emission. The lifetime of photoexcited electrons in the conduction band of bulk GaAs is long enough for spin relaxation mechanisms to have a large effect on polarization. A polarization of only $29 \%$ was measured in bulk GaAs at room temperature. Since photoexcited electrons spend a short time inside thin photocathodes, the spin relaxation mechanisms do not adversely affect the polarization during emission. A much better polarization of $44 \%$ was measured in thin GaAs at room temperature. Thus, spin relaxation mechanisms that have a strong temperature dependence do not seem to have a large effect on thin photocathodes.

\subsection{Polarization Sensitivity To Quantum Efficiency}

It has been observed that the polarization of a photocathode changes as the $\mathrm{QE}$ changes.[27] In particular; the polarization seems to improve as the $\mathrm{QE}$ decays. On the 
other hand, the polarization drops a few percentage points after each cesiation when the $\mathrm{QE}$ is near its highest value. This section will present the study of polarization around the cesiation time and it will explore some possible explanations as to why the polarization changes.

Figure 4.10 illustrates the polarization and $\mathrm{QE}$ of a strained $\mathrm{GaAs}$ photocathode before and after cesiation. In this particular instance cesium was deposited on the surface of the photocathode until the $\mathrm{QE}$ reached its maximum value and then more cesium was deposited until the $\mathrm{QE}$ dropped to its previous level of $0.42 \%$. This tẹchnique of

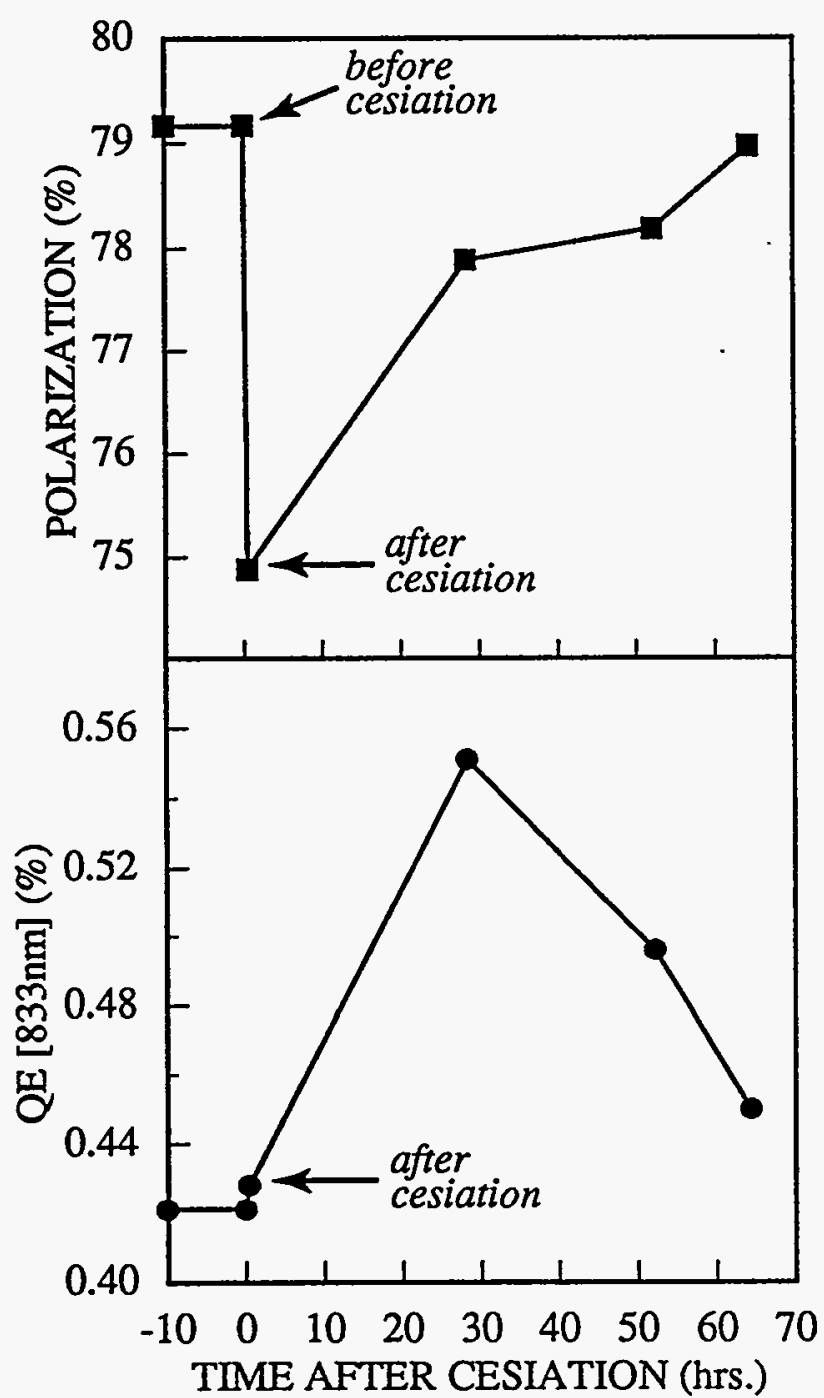

Figure 4.10: Polarization and $Q E$ of a strained GaAs photocathode before and after overcesiation. 
depositing extra cesium is called overcesiation and it is practiced frequently at the SLAC injector to increase the $\mathrm{QE}$ lifetime.[27] After the photocathode is overcesiated the $\mathrm{QE}$ typically rises and then it continues its normal time decay.

The top graph in Fig. 4.10 shows how the polarization drops after overcesiation even though the $\mathrm{QE}$ is at the relatively low level of $0.42 \%$. This behavior implies that the drop in polarization is due to the 'fresh' cesium on the photocathode surface. In order for the electrons to lose their spin orientation, they must be incurring a spin-exchange scattering with ionized atoms on the surface of the photocathode. After the excess cesium on the surface recombines with impurities and forms a new oxide layer, the $\mathrm{QE}$ returns to its high levels as shown in the bottom graph of Fig. 4.10. Note that as the QE rises, the polarization improves to a level close to where it was before cesiation. At this high $\mathrm{QE}$ level, the cesium-oxide layer does not seem to affect polarization in the same way that cesium did after overcesiation.

Figure 4.10 also shows that as the QE starts to decay, the polarization keeps improving. Why does the polarization improve at low QEs? The answer might lie in the higher vacuum level which occurs at low $\mathrm{QEs}$. The high vacuum level might be blocking the lower polarization electrons in the bottom of the conduction band. This idea is better illustrated in Fig. 4.11. Photoexcited electrons that reach the surface have a higher probability of getting reflected back into the band-bending region when the vacuum level is high. In section 2.3.2 it was described that an electron that spends more time inside the

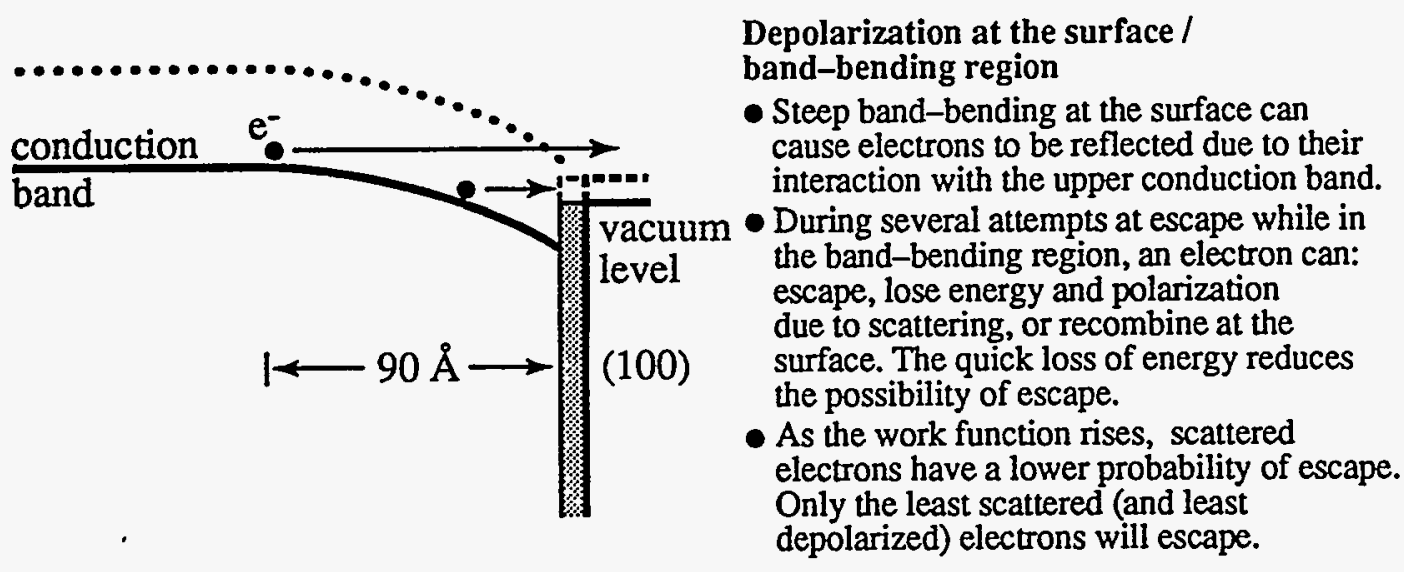

Figure 4.11: Depolarization at the surface / band-bending region. When the vacuum level rises, only the least scattered (and least depolarized) electrons escape. 
semiconductor is more likely to suffer spin relaxation and energy loss. Those electrons that lose too much energy will not be able to escape and will get trapped at the surface or recombine with the valence band. When the $\mathrm{QE}$ drops, the vacuum level rises and blocks those electrons that have scattered the most. Thus, only the least scattered (and least depolarized) electrons escape.

It has been observed that the phenomenon of higher polarization at lower QEs is more pronounced in strained GaAs photocathodes. Thus the high vacuum level and the photocathode strain are suspected to be closely related in the polarization sensitivity to $\mathrm{QE}$. Figure 4.12 illustrates the energy bands in GaAs for ideal strain and for partially relaxed strain. The actual photocathode is believed to be a combination of these two types of bands which results in the broadening of the energy bands. In the case of ideal strain, higher energy photons are required to excite electrons into the conduction band. Unfortunately, these high energy photons have a finite probability of exciting electrons of the wrong spin orientation into the conduction band, thus causing a drop in the overall polarization. Electrons photoexcited into the broader conduction band will have different polarization states, the electrons higher in the band will have higher polarization than those lower in the band. When the $\mathrm{QE}$ is high (i.e. the vacuum level is low), the electrons which are high and low in the conduction band will be able to escape, thus producing a lower polarization. As the $\mathrm{QE}$ decays, only those electrons higher in the conduction band will be able to escape, thus raising the polarization. This phenomenon is illustrated in Fig. 4.13.

In summary, the polarization sensitivity to $Q E$ is rather small, just a few percentage points. It is believed that when the photocathode has a low $\mathrm{QE}$, the high vacuum level

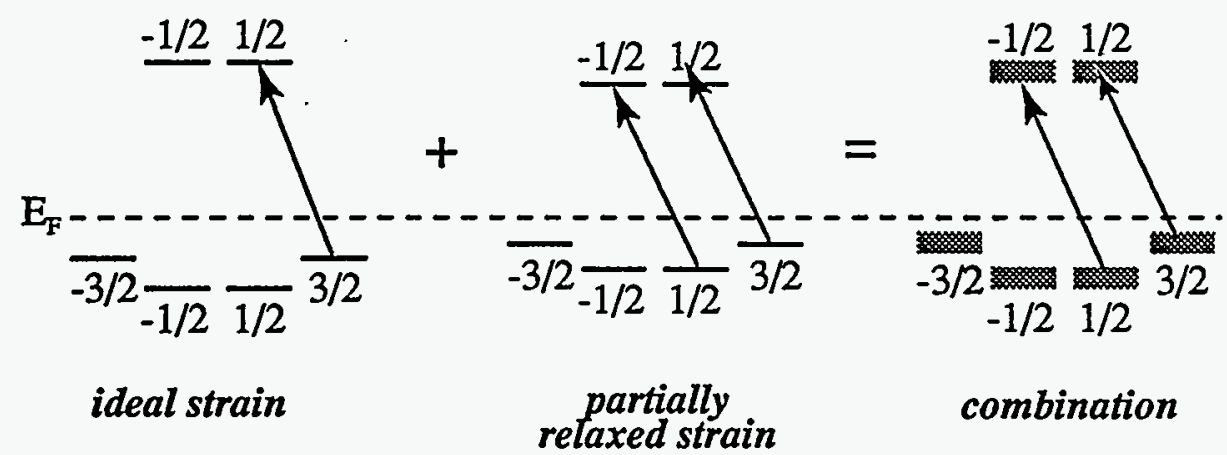

Figure 4.12: Effective energy band structure in strained GaAs photocathodes. The combination of regions of high strain with regions of relaxed strain results in a broadening of the valence and conduction bands. 


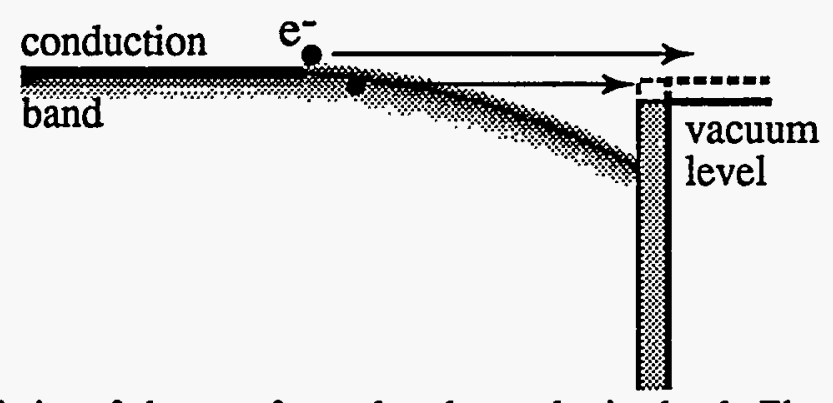

Figure 4.13: Photoemission of electrons from a broader conduction band. Electrons found higher in the band have higher polarization than those electrons found lower in the band. When the vacuum level rises, only those electrons high in the conduction band will escape, thus raising the overall polarization.

filters out the low polarization electrons thus producing slightly higher polarization beams. SLAC requires high intensity beams which in turn requires high $Q E$ values. This requirement results in a slightly smaller polarization than what is achievable.

\subsection{Charge Limit Effects}

In section 2.4.1 it was discussed that the maximum current density obtainable from an ideal cathode is given by Child's Law. In that derivation it was assumed that the cathode is an infinite source of electrons. In reality, however, the maximum current density obtainable from the SLAC photocathodes is lower than that predicted by Child's Law and it seems to depend on the photoemissive properties of the semiconductor. The maximum current density dependence on the semiconductor's photoemission properties is known as the semiconductor charge limit (CL) effect. As discussed in section 2.4.2, the CL depends on the surface properties of the semiconductor. The surface properties of a photocathode change rather quickly during the production of intense electron beams. This section will present data illustrating the different manifestations of the $\mathrm{CL}$ and then it will try to establish a physical basis for this phenomenon.

One of the first indications of a $\mathrm{CL}$ in GaAs photocathodes was the observation that the maximum emitted current density increased with higher values of $\mathrm{QE}$.[3] This behavior is illustrated in Fig. 4.14 for a $14 \mathrm{~mm}$ diameter bulk GaAs photocathode. The electron bunch was produced by a $2.5 \mathrm{~ns}$ FWHM, $720 \mathrm{~nm}$ Ti:Sapphire laser pulse. The QE 


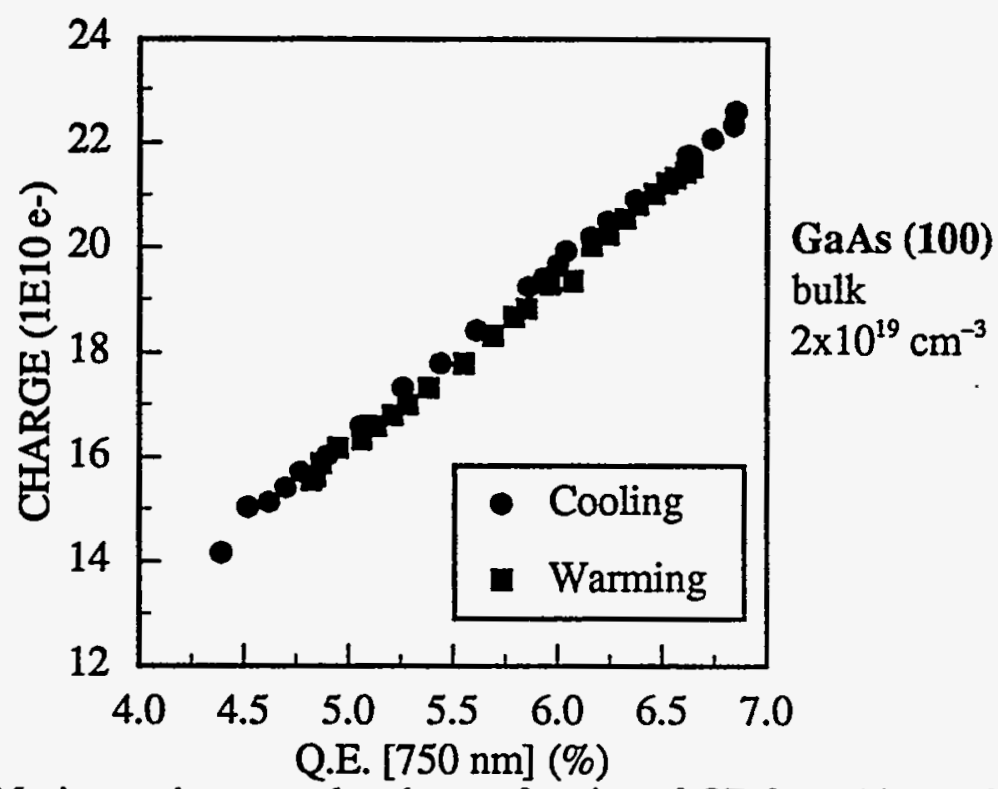

Figure 4.14: Maximum charge per bunch as a function of $\mathrm{QE}$ for a $14 \mathrm{~mm}$ diameter bulk GaAs photocathode. The QE was increased by lowering the temperature of the photocathode. The round points indicate when the photocathode was being cooled and the square points indicate when the photocathode was being warmed.

(measured at $750 \mathrm{~nm}$ illumination) was increased by lowering the photocathode temperature (see section 4.2). The plot in Fig. 4.14 shows a nearly linear relationship between the maximum emitted charge and $\mathrm{QE}$.

Another manifestation of the $\mathrm{CL}$ is the change in the temporal profile (i.e. the charge versus time plot) of an electron bunch. Figure 4.15 shows a GAPM oscilloscope scan of a severely charge limited electron bunch. The FWHM of the electron bunch is only $\sim 500$ ps while the FWHM of the laser pulse is $\sim 2 \mathrm{~ns}$. This behavior indicates that at very low $\mathrm{QE}$ and high laser illumination the $\mathrm{CL}$ can shut off photoemission.

Yet another interesting manifestation of the $\mathrm{CL}$ is observed during the production of a train of bunches. Figure 4.16 shows two bunches, initially separated in time by $60 \mathrm{~ns}$, with the second bunch being moved closer to the first bunch. The maximum charge in the second bunch decreases as it is moved closer to the first bunch. This behavior indicates that when a photocathode is illuminated into CL, it requires some time to return to 'normal' operation. It will be shown later in this section that all the above manifestations of charge saturation give clues about the physical basis of the CL phenomenon. 


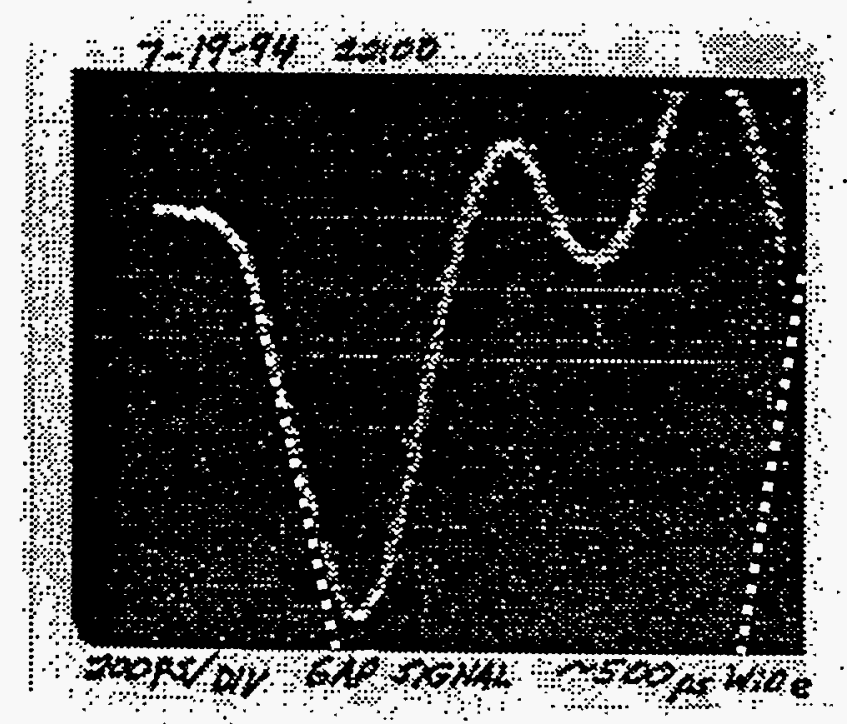

Figure 4.15: Temporal profile of a severely charge-limited electron bunch. The profile was obtained with the GAPM where the voltage signal is proportional to charge in the electron bunch. The white dashed line depicts a 2 ns FWHM electron bunch. The ringing in the GAPM signal is due to the mismatched transmission line.

The first step towards understanding the physical basis of the $\mathrm{CL}$ is to look at the photoemission process when it has a linear response to the incident light and then try to find out what changes take place in the semiconductor during periods of high intensity illumination. To simplify the discussion on the CL phenomena, it is easier to look at the photoemission process for continuous illumination. The equation that describes the relationship between the various electron current densities during the photoemission process can be found by utilizing charge conservation

$$
J_{0}=J_{e}+J_{R}+J_{S}
$$

where $J_{0}$ is the current density of electrons being photoexcited from the valence band into the conduction band, $J_{e}$ is the emitted current density, $J_{R}$ is the current density for electrons recombining to the valence band, and $J_{S}$ is the current density being trapped at the photocathode surface. Equation 4.9 says that as the photocathode is being illuminated, a continuous number of electrons are being excited from the valence band into the conduction 


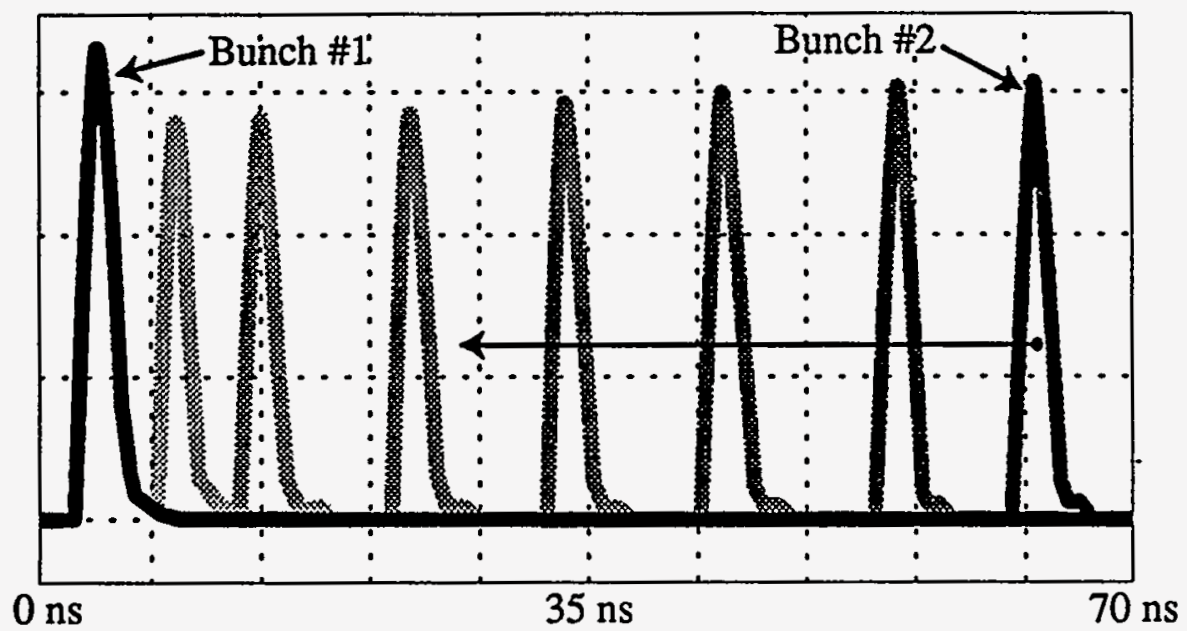

Figure 4.16: Effect of bunch \#1 on the maximum charge in bunch \#2 (bunch-bunch effect). As bunch \#2 is moved closer in time to bunch \#1, the maximum charge in bunch \#2 decreases. This illustration was adapted from an actual GAPM oscilloscope signal.

band and that these excited electrons can either escape, recombine to the valence band, or get trapped at the surface.

When the photoemission is in linear mode, the emitted current density is proportional to the excited current density (which in turns is proportional to the incident photon intensity). This proportionality is described by

$$
J_{e} \propto p_{e} J_{0}
$$

where $p_{e}$ is the probability that a photoexcited electron will escape once it reaches the surface. In addition, the rate at which the charge gets trapped at the surface is the same as the rate at which the surface charge recombines with the valence band. This relationship is described by

$$
J_{r}=J_{s}
$$

where $J_{r}$ is the current density that describes the recombination from the surface states into the valence band. The linear photoemission process is illustrated in Fig. 4.17. 


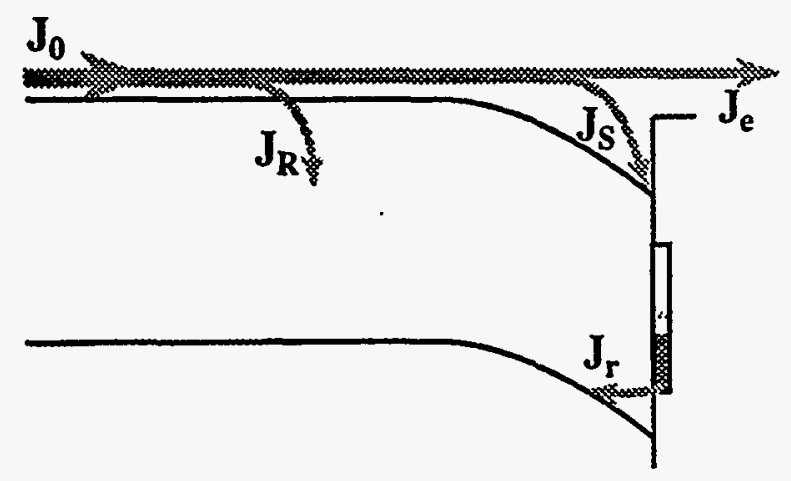

Linear-mode equations

$$
\begin{gathered}
\mathbf{J}_{\mathbf{0}}=\mathrm{J}_{\mathrm{e}}+\mathrm{J}_{\mathrm{R}}+\mathrm{J}_{\mathrm{S}} \\
\mathrm{J}_{\mathrm{e}} \propto \mathbf{p}_{\mathrm{e}} \mathrm{J}_{\mathbf{0}} \\
\mathrm{J}_{\mathrm{r}}=\mathrm{J}_{\mathrm{S}}
\end{gathered}
$$

Figure 4.17: Energy band diagram illustrating a semiconductor undergoing linear response photoemission. The linear-mode equations include: the current conservation, the proportionality between the excited and emitted current densities, and the equality of the current trapped at the surface and the current that recombines with the valence band.

The photoemission process deviates from its linear behavior when the density. of photoexcited electrons is increased. In particular, the changes in photoemission occur when the current density getting trapped in the surface states surpasses the current density recombining to the valence band, or simply put, when $J_{S}>J_{r}$. When this situation occurs, charge accumulates at the surface states which in turns raises the surface potential barrier. The rate of change of the trapped charge density at the surface $\sigma_{T}$ is described by

$$
\frac{d \sigma_{T}}{d t}=J_{S}-J_{r}
$$

The rise in the surface potential barrier reduces the probability of escape of photoexcited electrons and thus reduces the emitted current density. The emitted current density then becomes a complicated function of time

$$
J_{\iota} \propto p_{\varepsilon}(t) J_{0}
$$

The photoemission process for a saturated photocathode is illustrated in Fig. 4.18. It is important to note that the surface recombination current density $J_{r}$ is larger in high-doped semiconductors.[26] The larger surface recombination current density occurs because the trapped surface electrons have a higher probability of tunneling across the narrower depletion region (for a review of the depletion region see section 2.1.5). Since the 


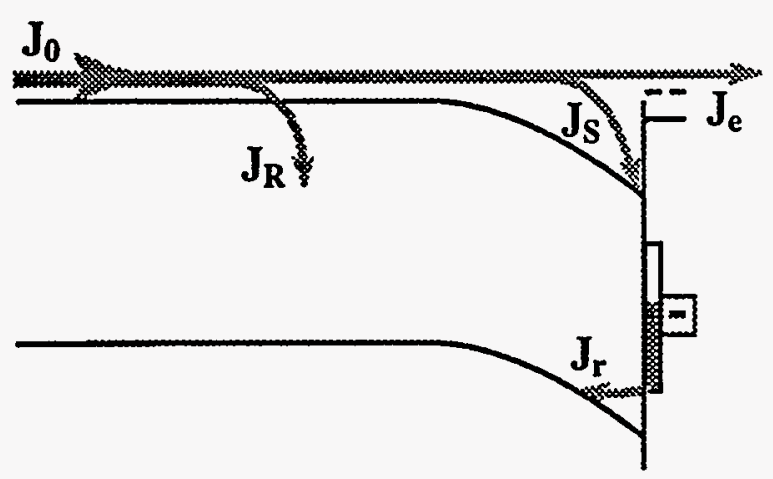

\section{$\underline{\text { Saturation-mode equations }}$ \\ $\mathbf{J}_{\mathbf{S}}>\mathbf{J}_{\mathbf{r}}$ \\ $\frac{\mathrm{d} \sigma_{\mathrm{T}}}{\mathrm{dt}}=\mathrm{J}_{\mathrm{S}}-\mathbf{J}_{\mathbf{r}}$ \\ $\mathbf{J}_{\mathrm{e}} \propto \mathbf{p}_{\mathrm{e}}(\mathbf{t}) \mathbf{J}_{0}$}

Figure 4.18: Energy band diagram illustrating a semiconductor with diminishing photoemission yield. Note in particular that the negative charge that accumulates at the photocathode surface raises the surface potential barrier. The saturation-mode equations include: the inequality between the current density of electrons getting trapped at the surface and the current density of electrons recombining to the valence band, the rate of change of charge density trapped at the surface, and the nonlinear relationship between emitted current density and the excitation current density.

maximum $J_{r}$ is larger in high-doped semiconductors, it takes a higher $J_{S}$ to reach saturation. For this reason, high-doped photocathodes achieve larger emitted current densities before reaching saturation than low-doped photocathodes.

The current conservation Eqn. 4.9 and the saturation-mode Eqns. 4.12 and 4.13 seem to correctly explain the CL behavior observed in Figs. 4.14, 4.15, and 4.16. For instance, if the probability of escape is high, a higher current density is going to be emitted and a smaller current density is going to get trapped at the surface. For this reason, higher QE photocathodes yield larger maximum emitted current densities than low QE photocathodes, as was shown in Fig. 4.14. On the other hand, low QE photocathodes have lower emitted current densities and higher charge trapped at the surface. This trapped charge raises the surface potential barrier and shut off photoemission as was shown in Fig. 4.15. Finally, the electrons trapped in the surface states will eventually recombine with the holes in the valence band. However, since the surface recombination velocity is finite (and probably has some type of time dependence), the surface potential barrier will decrease with time as if it had a decay constant. This behavior was observed in Fig. 4.16 as the maximum charge of the second bunch increased as it was moved away in time from the first bunch.

A limit in the surface recombination current density $J_{r}$ can have serious consequences on the maximum current density available from these photocathodes, 
especially for a wavelength of illumination close to the bandgap. This observation was made by Professor Roger Miller during the defense of this dissertation. It appears that if the surface recombination current density has an upper limit, then a high enough illumination intensity will cause enough trapping of electrons at the surface that will shutoff photoemission. The illumination intensity necessary to shut off photoemission can be raised by increasing the probability of escape at the surface. Another complication arising from the upper limit in the surface recombination current is that the maximum emitted current density will depend on the rate of creation of electron-hole pairs (which is determined by the laser pulse profile). The reason for this complication will be discussed later on in this section.

An important clue which indicates that the emitted current density has an upper limit is that the photocathodes tend to saturate once a given number of electron-hole pairs have been created. This behavior can be seen in Figs. 4.19 and 4.20. This data was taken by Huan Tang in the GTL in '93.[47] These figures show saturation plots (i.e. maximum emitted charge versus laser intensity) at two different wavelengths for various $Q E$ values. The vertical lines indicate the laser intensity which produces the maximum charge per pulse. Note that this maximum occurs roughly at the same laser intensity for various $Q E$

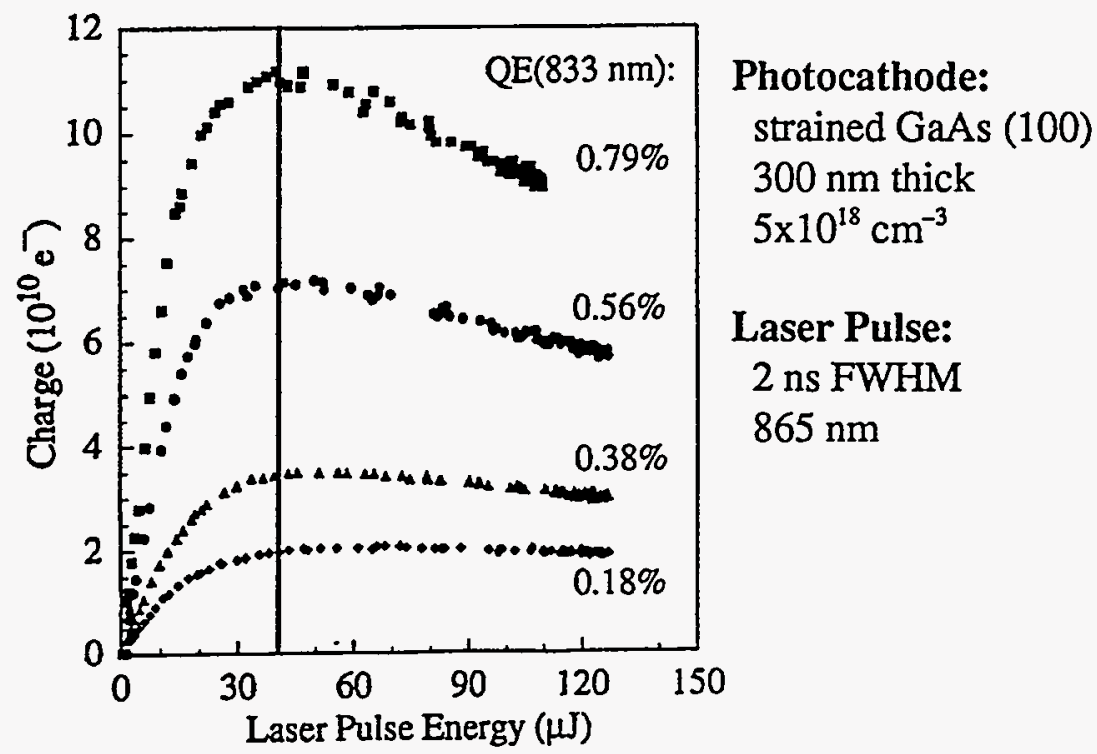

Figure 4.19: Saturation plots of $300 \mathrm{~nm}$ strained $\mathrm{GaAs}$ for various $\mathrm{QE}$ levels.[47] The vertical line indicates roughly the laser intensity at which the maximum charge per pulse occurs. 
levels. This behavior implies that after a large number of electrons are photoexcited into the conduction band, many of them will get trapped at the surface and fill the surface states. The filled surface states will in turn raise the surface potential barrier and prevent further photoemission.

Note that in Figs. 4.19 and 4.20 the laser intensity that saturates the photocathode changes with wavelength. The reason for this behavior is that the production of electronhole pairs has a very strong wavelength dependence. For instance, shorter wavelength photons are absorbed much more efficiently than longer wavelength photons. Therefore, shorter wavelength illumination requires less photons to achieve saturation. In Fig. 4.19 the charge production saturates for a laser pulse intensity of $40 \mu \mathrm{J} /\left(\pi^{*} \mathrm{~cm}^{2}\right) /$ pulse or, for $865 \mathrm{~nm}$ illumination, $5.5 \times 10^{13}$ photons $/ \mathrm{cm}^{2} /$ pulse. In Fig. 4.20 the charge production saturates for a laser pulse intensity of $10 \mu \mathrm{J} /\left(\pi^{*} \mathrm{~cm}^{2}\right) /$ pulse or, for $775 \mathrm{~nm}$ illumination, $1.24 \times 10^{13}$ photons $/ \mathrm{cm}^{2} / \mathrm{pulse}$. Since the shorter wavelength photons are absorbed more efficiently than the longer wavelength photons, $1.24 \times 10^{13} \mathrm{pairs} / \mathrm{cm}^{2} / \mathrm{pulse}$ is closer to the actual number of electron-hole pairs created in the active region of the photocathode rather than the $5.5 \times 10^{13}$ pairs $/ \mathrm{cm}^{2} /$ pulse. Since about $30 \%$ of the incident light is reflected in GaAs [48], the number of electron-hole pairs created is closer to $8.7 \times 10^{12}$ pairs $/ \mathrm{cm}^{2} / \mathrm{pulse}$. This number is very close to the positive surface charge density of $4.5 \times 10^{12} \mathrm{e} / \mathrm{cm}^{2}$ due to

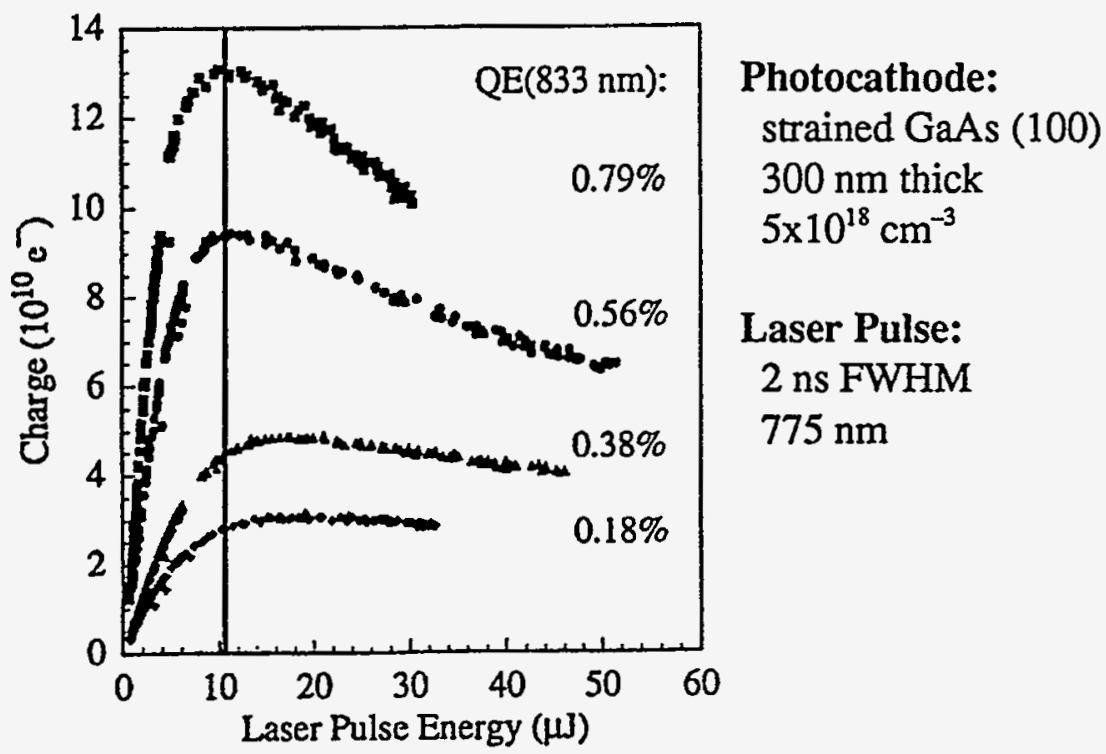

Figure 4.20: Saturation plots of $300 \mathrm{~nm}$ strained GaAs for various QE levels.[47] The vertical line indicates roughly the laser intensity at which the maximum charge per pulse occurs. 
the depletion region (refer to section 2.1.5). Since less than $1 \%$ of photoexcited electrons get emitted, the majority will reach the surface and get trapped there. This is especially the case in thin photocathodes because electrons cannot diffuse into the bulk. Thus the experimental evidence suggests that when $10^{12}-10^{13} \mathrm{e}^{-} / \mathrm{cm}^{2}$ reach the surface in a very short period of time, the photoemission properties of the photocathode change dramatically and induce charge saturation.

A very interesting phenomenon is the relationship between the emitted charge density and the shape of the laser pulse. For instance, it has been observed that a large emission current density can be obtained if the laser pulse is short and intense. On the other hand, if the laser pulse is long and of medium intensity, the maximum charge per bunch is going to be lower because charge had been building up at the photocathode surface before the laser pulse was completed. Thus to get the most charge out of a photocathode, it helps to obtain the highest $\mathrm{QE}$ possible (through good activation or very high voltages) and to make the laser pulses shorter and very intense. This technique basically causes a large photoexcited current density to hit the low vacuum level. Figure

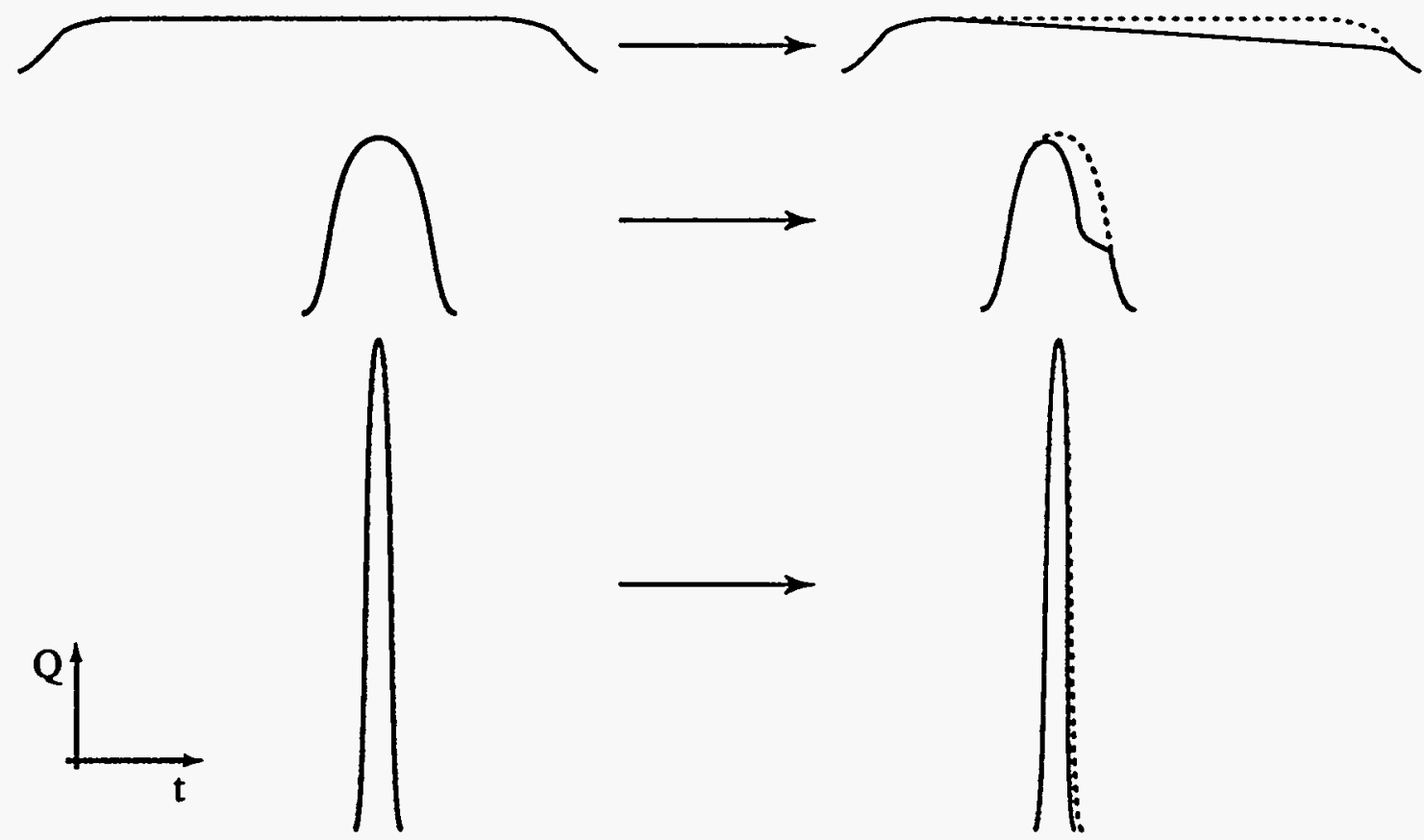

Figure 4.21: Effect of the CL on laser pulses with different time profiles. The figures on the left represent the photoexcited charge distribution vs. time in the conduction band. The figures on the right represent the emitted charge distribution. There is a clear advantage in utilizing shorter time pulses because a higher charge intensity sees a high probability of escape. 
4.21 illustrates the relationship between the emitted charge and the shape of the laser pulse.

The rest of this section will attempt to derive a simple expression that describes the maximum emitted current density available from the SLAC GaAs photocathodes. Since this current density varies with $\mathrm{QE}$ and since it peaks at a certain laser intensity, the expression for the maximum current density must be a function of the probability of escape and of the density of surface states. The probability of escape depends strongly on the vacuum level which in itself depends on the impurity caused band-bending potential and on the Cs-F layer deposited at the surface. Both of these quantities, the doping concentration and the Cs-F layer, remain constant during the photoemission process. However, the number of electrons trapped at the surface does vary during photoemission and it has an effect on the probability of escape. When a certain amount of charge is trapped at the surface, the energy bands unbend and the vacuum level rises thus reducing significantly the probability of escape. The time that it takes the surface to return to its normal bandbending state depends on the surface recombination velocity (which in turns depends strongly on the doping concentration). The amount of charge trapped at the surface that is required to raise the vacuum level is of the same order of magnitude as the surface charge density created by the depletion region. This surface charge density was obtained in section 2.1.5 and is given by

$$
\sigma_{s}=q N_{a} w
$$

For $N_{a}=5 \times 10^{18} \mathrm{~cm}^{-3}$ and $w \approx 90 \AA$, the approximate surface charge density in GaAs is $\sigma_{\mathrm{s}}$ $\approx 4.5 \times 10^{12} \mathrm{e}^{-} / \mathrm{cm}^{2}$.

To derive the maximum emitted current density in III-V semiconductor photocathodes, it is natural to start at the point when the photoemission is linear with respect to the intensity of illumination. If $J_{0}$ is the current density reaching the surface (for thin photocathodes it is a good approximation to assume that almost all photoexcited electrons reach the surface), then the current density of emitted electrons is given by

$$
J_{e}=p_{e} J_{0}
$$


where $p_{c}$ is the probability of escape. The current that is not emitted is the current that recombines with the valence band plus the current that gets trapped at the surface and is given by

$$
J_{R}+J_{S}=\left(1-p_{e}\right) J_{0}
$$

Dividing Eqn. 4.15 by Eqn. 4.16 and solving for $J_{e}$ results in

$$
J_{e}=\frac{p_{e}}{1-p_{e}}\left(J_{R}+J_{S}\right)
$$

Note that $J_{\mathrm{e}}$ tends to zero when $p_{\mathrm{c}}$ tends to zero. The limit of $J_{e}$ as $p_{e}$ tends to one is, of course, $J_{0}$. Equation 4.17 becomes more complicated during charge saturation when $p_{e}$ is a function of time.

The maximum charge per pulse obtainable from GaAs photocathodes is difficult to compute because the probability of escape changes during saturation and because the intensity of the laser pulse is not constant. However, a good estimate of the maximum charge per pulse can be obtained by making some approximations of the various current densities. First, the majority of photoexcited electrons in thin photocathodes get either emitted or trapped at the surface rather than recombining with the valence band. Thus $J_{R} \sim$ 0 . This is a reasonable assumption because the active region in thin photocathodes is much shorter than the electron diffusion length. Second, if the laser pulse is intense enough such that $J_{S} \gg J_{r}$ then the maximum time that the photoemission is 'ON' is obtained from

$$
J_{S} \Delta t_{\max }=\sigma_{\text {limit }}
$$

where $\sigma_{l i m i}$ is $\sim 4.5 \times 10^{12} \mathrm{e}^{-} / \mathrm{cm}^{2}$. Thus the maximum emitted charge density is given by

$$
\sigma_{\text {emitred,max }}=J_{e} \Delta t_{\max }=\frac{J_{e}}{J_{S}} \sigma_{\text {limit }}
$$

Substituting Eqn. 4.17 into Eqn. 4.19 results in 


$$
\sigma_{\text {emitted,max }}=\frac{p_{e}}{1-p_{e}} \sigma_{\text {limit }}
$$

For a $20 \mathrm{~mm}$ diameter photocathode, Eqn. 4.20 simplifies to

$$
Q_{\text {emitted, } \max }=\frac{p_{e}}{1-p_{e}} 1.6 \times 10^{13} e^{-}
$$

An example of the maximum emitted charge density can be obtained in the case of a $100 \mathrm{~nm}$ strained $\mathrm{GaAs}$ photocathode with a $\mathrm{QE}$ of $0.6 \%$ at $841 \mathrm{~nm}$. Assuming that $p_{e} \sim \mathrm{QE}$ for thin photocathodes, Eqn. 4.21 then yields $Q_{\text {emitted } \max }=9.7 \times 10^{10} \mathrm{e}^{-}$. This result is quite close to the actual laboratory number of $\sim 1 \times 10^{11} \mathrm{e}^{-}$. Equation 4.21 implies that the maximum emitted charge has no upper limit as $p_{c}$ tends to one. In reality, however, the probability of escape that can be obtained in the laboratory is much less than one, especially for thin photocathodes. Finally, Chapter 5 will discuss how the maximum emitted current density can be improved by increasing the doping concentration, by adding a conductive layer to the surface, and by selecting a different crystal orientation. These improvement would require the modification of Eqns. 4.18 and 4.20.

\subsection{Polarization Sensitivity to Laser Intensity}

One reason for building the Mott polarimeter was to investigate the effect that the CL can have on the beam polarization. The experience at the SLAC injector had shown that a fully illuminated photocathode under charge saturation would show no drop in polarization. Actually, since most photocathodes under strong charge saturation have low QEs, the polarization would be relatively high. In the GTL a similar photocathode was illuminated with a small and highly intense laser pulse and, to everybody's surprise, the polarization dropped. Moreover, when the photocathode was fully illuminated, the polarization did not drop, just like in the SLAC injector. This section will present data illustrating this newly 


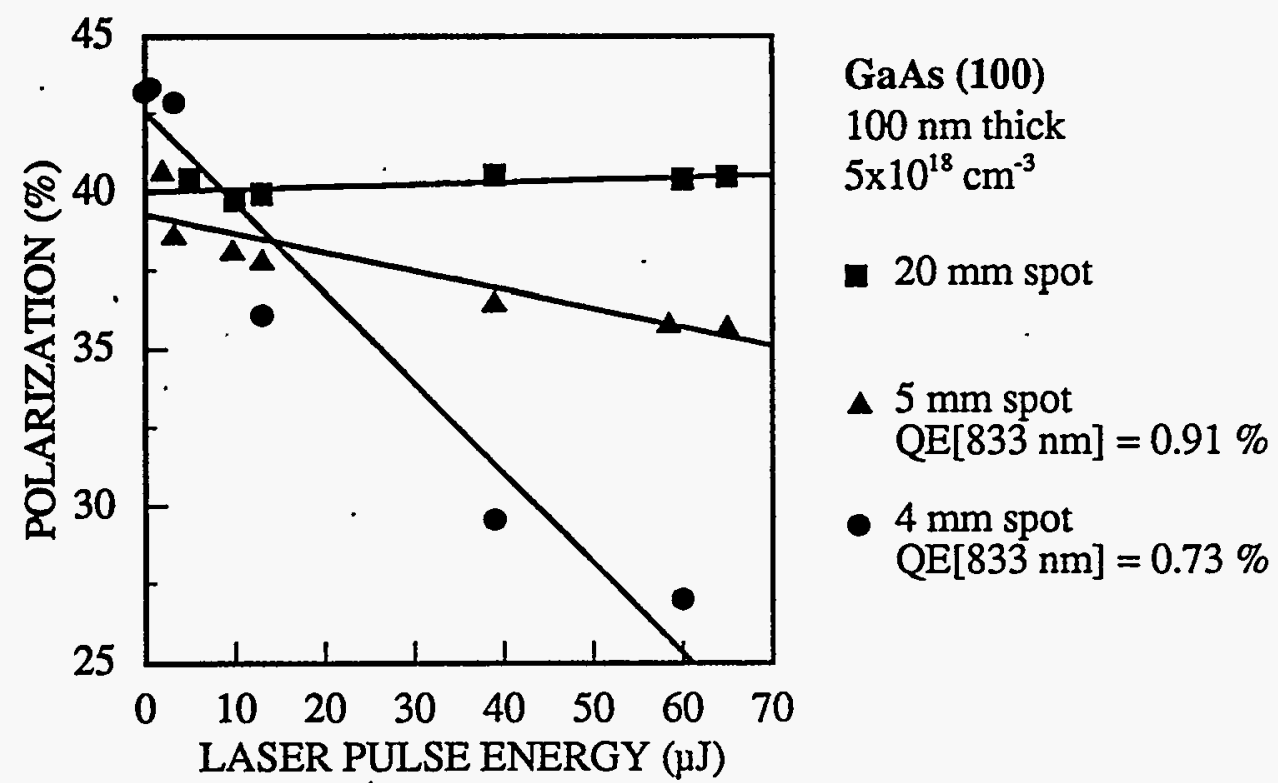

Figure 4.22: Polarization dependence on laser intensity for different laser spot sizes. Also included are the QEs for the smaller laser spots.

found polarization dependence on laser intensity and it will present some ideas that try to explain this phenomenon.

The observation of lower polarization with high intensity illumination was seen in all the photocathodes, from the standard GaAs photocathode to the $100 \mathrm{~nm}$ strained GaAs. Figure 4.22 shows the change in polarization as a function of laser intensity for three different spot sizes in the standard GaAs photocathode. Note that the polarization for a fully illuminated photocathode does not decrease with increasing laser pulse intensity. However, the polarization can drop considerably for the smaller laser spots. Another example of the drop in laser polarization at high laser pulse intensities is shown in Fig. 4.23 for a $200 \mathrm{~nm}$ strained GaAs photocathode. The polarization at low laser intensities is not as high as it could have been for this photocathode because the laser wavelength was tuned to $841 \mathrm{~nm}$ instead of the optimum $875 \mathrm{~nm}$. Also included in Fig. 4.23 is a photocathode profile illustrating the small laser spot.

When a photocathode is illuminated with a small laser spot into charge saturation, the charge in the bunch increases with higher laser intensities. Figure 4.24 illustrates the electron bunch profile as the $1 \mathrm{~mm}$ laser spot intensity is increased. Also shown in Fig. 4.24 is the polarization for each electron bunch. The figure includes three oscilloscope 


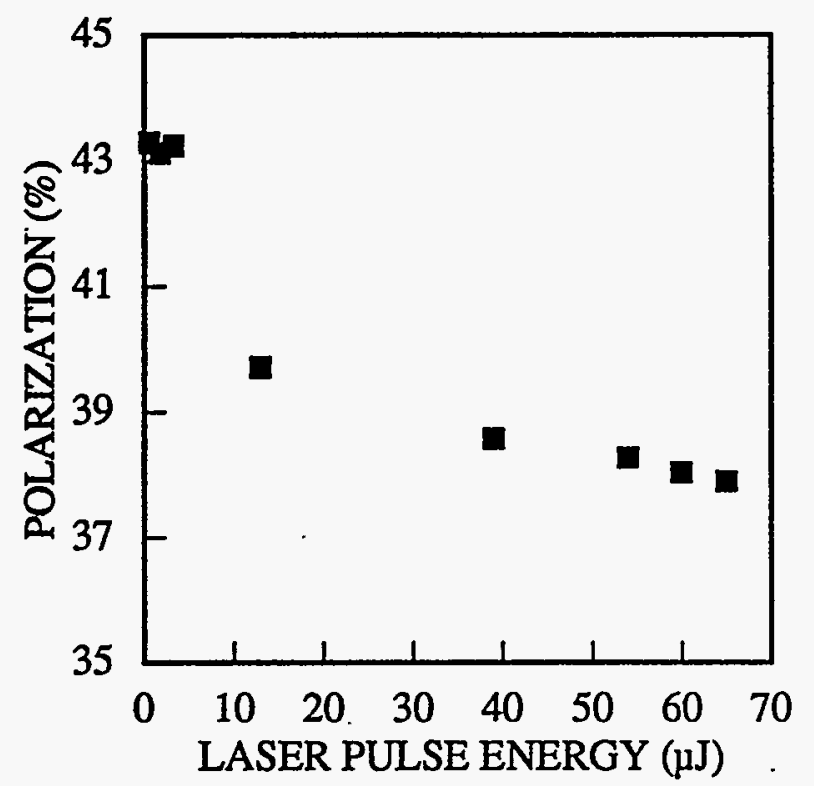

strained GaAs (100)

$200 \mathrm{~nm}$ thick

$5 \times 10^{18} \mathrm{~cm}^{-3}$

$1 \mathrm{~mm}$ spot

$\mathrm{QE}[833 \mathrm{~nm}]=0.87 \%$

$\lambda=841 \mathrm{~nm}$

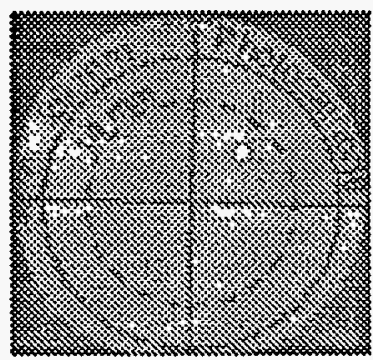

Figure 4.23: Polarization dependence on laser intensity for a $200 \mathrm{~nm}$ strained GaAs photocathode. The laser wavelength for optimum polarization is $875 \mathrm{~nm}$, not the $841 \mathrm{~nm}$ used in these measurements. Also shown is a photocathode profile illustrating the small laser spot.

screens with either two or three superimposed images. The vertical scale in the oscilloscope was adjusted between screens to accommodate the higher intensity bunches. The first electron bunch in screen (a) has the 2 ns FWHM shape of the low intensity laser pulse. The second electron bunch in screen (a) already shows signs of $\mathrm{CL}$ in its narrower profile (i.e. photoemission is being cutoff). Screen (b) shows two bunches with increasing charge but decreasing polarization. Where is this extra charge coming from if the semiconductor is already saturated? When the laser intensity is increased, the higher number of photons in the tails of the laser Gaussian profile will produce photoemission in the perimeter region of the semiconductor which is not saturated. Screen (c) shows four more bunches with increasing charge but decreasing polarization.

The results shown in Fig. 4.24 suggest that if the center region of the illuminated area of the photocathode is saturated so that no further photoemission is occurring, then the photoemission responsible for the drop in polarization is occurring at the outer edges of the illuminated area. If this is indeed the case, a nonuniform polarization distribution across the electron bunch is expected with the highest polarization occurring at the center of the bunch and the lowest polarization occurring around the edges of the bunch. Even though a 


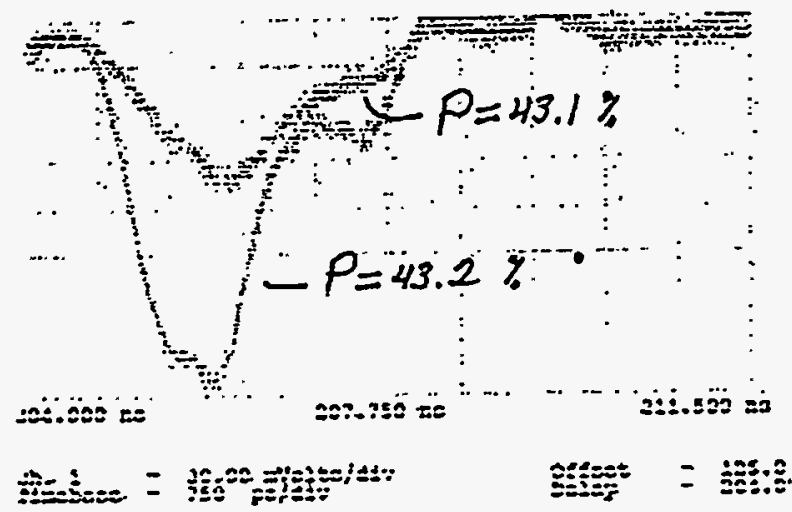

(a)

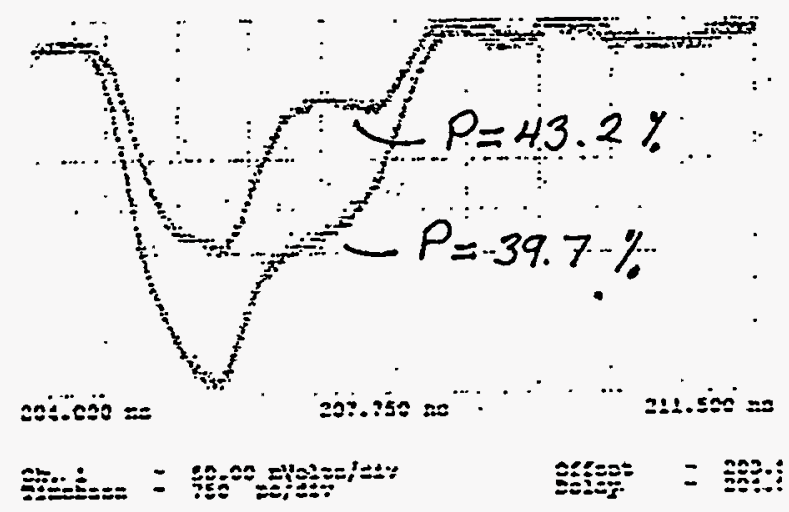

(b)

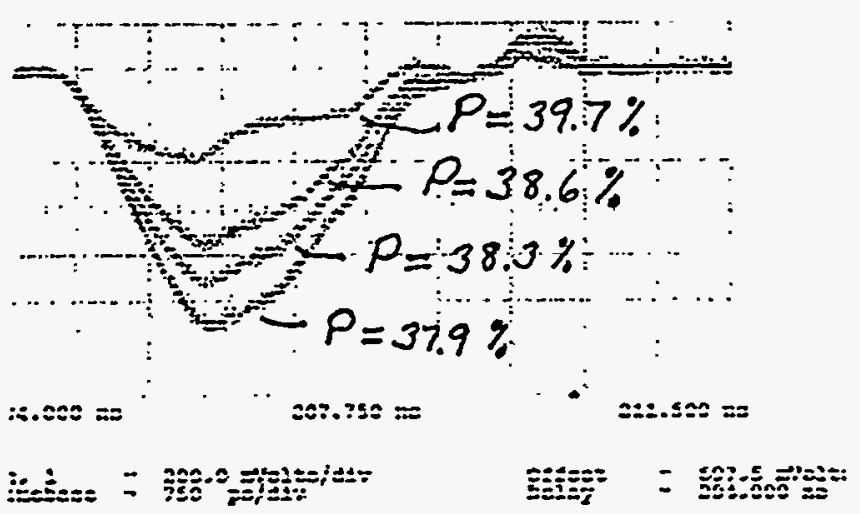

Figure 4.24: Electron bunch profile and polarization for a $1 \mathrm{~mm}$ laser spot with increasing intensity. In screens (b) and (c) the vertical scale of the oscilloscope was adjusted to accommodate larger bunches. For comparison purposes, the last bunch of screen (a) was reinserted as the first bunch of screen (b) and the last bunch of screen (b) was reinserted as the first bunch of screen (c). 
precise region of the electron bunch cannot be tested for polarization, the electron bunch can be scanned across the FFC aperture and the polarization of the passing beam can be measured. The results of such a test are shown in Fig. 4.25. First, a polarization of $69 \%$ was measured for a $1 \mathrm{~mm}$ electron beam from a region in the photocathode with very high $\mathrm{QE}$. The photocathode was not in saturation. Then the laser pulse intensity was increased and the resulting beam was scanned across the FFC aperture. The polarization measurements were below the nominal value of $69 \%$ and they varied depending on the scanned section of the bunch. These results indicate that the beam cross-section does not have uniform polarization. Unfortunately, this test cannot tell conclusively which area of the photocathode was producing the low polarization.

The obvious question that arises from the above experiments is whether the drop in polarization at higher laser intensities is due to a property of the semiconductor or whether it arises from instrumental causes, such as depolarization of the laser pulse itself. To try to answer this question, the following list of observations was compiled:

- The beam polarization is seen to drop by as much as $15 \%$ (absolute) when the laser intensity is on the order of $100 \mu \mathrm{J} / \mathrm{mm}^{2}$.

- The beam polarization does not drop when the photocathode is fully illuminated, in

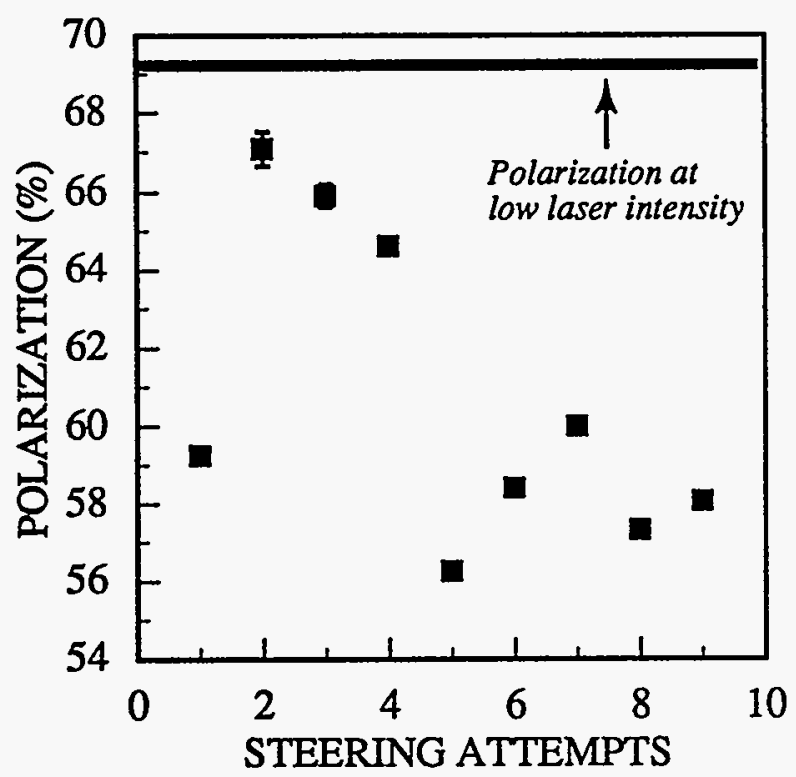

strained GaAs (100)

$200 \mathrm{~nm}$ thick

$5 \times 10^{18} \mathrm{~cm}^{-3}$

$1 \mathrm{~mm}$ spot

$\mathrm{QE}[833 \mathrm{~nm}]=0.80 \%$

$\lambda=875 \mathrm{~nm}$

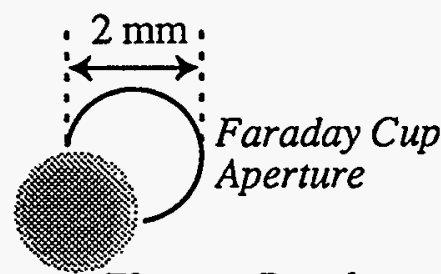

Electron Bunch

STEERING ATTEMPTS

Figure 4.25: Polarization of a charge-limited electron bunch scanned across the FFC aperture. The results indicate that the cross section of the electron beam does not have uniform polarization. 
agreement with observations at the SLAC injector.

- The beam polarization drops when the same high-intensity laser pulse that illuminated the full photocathode is misteered and only illuminates part of the photocathode.

- The beam polarization at high laser intensities is extremely dependent on what part of the bunch is transmitted along the FFC aperture.

- The polarization drop is more likely to occur, and in a larger magnitude, around the high $\mathrm{QE}$ areas rather than on the low $\mathrm{QE}$ areas of the photocathode.

Joseph Frisch suggested that the drop in polarization at high laser intensities is caused by nonuniform polarization across the laser pulse. His idea was that a fraction of the light going though the lenses scatters with imperfections and dust particles and loses polarization. The scattered light tends to diverge away from the beam path because it does not have the same momentum vector as the non-scattered light. As the laser intensity is increased, the center portion of the illuminated area on the photocathode becomes saturated and it can no longer emit electrons. The outer edge of the laser pulse now becomes responsible for the photoemission. However, the outer edge of the laser pulse has lower polarization because it is made up mostly of scattered photons. Thus, electrons created by the outer edge of the laser pulse have lower polarization.

One problem with the above hypothesis is that it is very hard to prove because the polarization across the laser pulse is difficult to measure. A rough attempt by Joseph Frisch and Ray Alley to measure the polarization across the laser pulse yielded no definitive results. Moreover, Frisch also suggested the possibility that the Pockels Cell could produce a nonuniform polarization. However, when the Pockels cell was substituted with a linear polarizer and a quarter-wave plate to produce the circular polarization, the polarization results did not change.

Another possible source for the low polarization at high laser intensities is the reflected laser light off the photocathode. Ignoring the Cs-F monolayer, the reflectivity of GaAs for wavelengths shorter than $2 \mu \mathrm{m}$ is about 30\%.[48] Most of the reflected light is believed to be directed backward but some of it is expected to be reflected isotropically inside the gun. The reflected light is expected to have low polarization and can conceivably 
be responsible for the lower polarization photoemission. Unfortunately, it is very difficult to determine what fraction of the charge in the bunch was produced by the reflected light.

There is still one more possible source for the low beam polarization at high laser intensities, the semiconductor itself. It was shown in section 2.3.2 that photoexcited electrons that spend a long time inside the semiconductor (such as in bulk GaAs) have lower polarization when they are emitted. In a similar way, electrons that have been photoexcited to the conduction band and cannot escape due to a higher surface potential barrier will spend a longer time in the conduction band, undergo spin relaxation, and eventually recombine back to the valence band. However, photoexcited electrons near the edge of the laser pulse can conceivably diffuse around the high potential barrier and still manage to escape. This phenomenon is illustrated in Fig. 4.26.

Electrons photoexcited behind the high surface potential barrier must move radially outward in order to encounter a low surface potential barrier and escape. The energy band diagram in Fig. 4.26 suggests that electrons in the saturated region will experience large repulsion from the negative charge trapped in the surface states. Thus photoexcited electrons reaching the surface will move radially outward from this electrical repulsion and
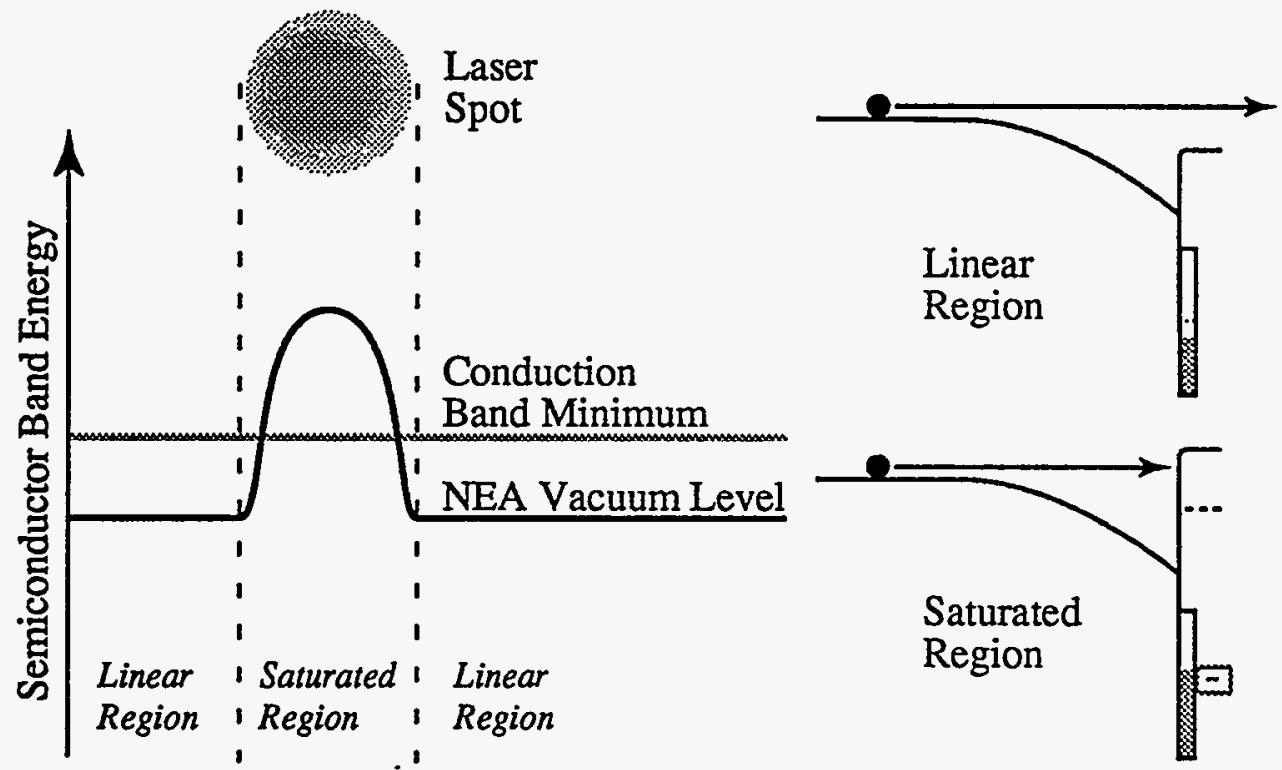

Figure 4.26: Vacuum level in a semiconductor experiencing CL. The figure on the left shows the vacuum level above the conduction band minimum in the saturated region. Electrons that drift towards the nonsaturated region around the laser spot have a chance to escape. The two figures on the right illustrate the energy band diagrams for the linear and saturated regions of the photocathode. 
also from plain random scattering. Can photoexcited electrons reach the outer edges of illumination before they recombine to the valence band or before they get trapped at the surface? A rough estimate of the distance of radial travel can be obtained by considering the motion of a 'ballistic' electron with kinetic energy given by the band-bending potential. For GaAs photocathodes with a doping concentration of $5 \times 10^{18} \mathrm{~cm}^{-3}$ this kinetic energy is given by

$$
\frac{1}{2} m^{*} v^{2} \approx 250 \mathrm{meV}
$$

where $m^{*}$ is the effective mass of the electron in the GaAs semiconductor.[10] If the electron lifetime before recombination is on the order of $3 \times 10^{-10} \mathrm{~s}$, then the distance of radial travel is approximately

$$
l_{\text {radial }}=v \cdot \tau=1.1 \times 10^{8} \frac{\mathrm{cm}}{\mathrm{s}} \cdot 3 \times 10^{-10} \mathrm{~s}
$$

or

$$
l_{\text {radial }}=0.3 \mathrm{~mm}
$$

where the velocity $v$ was obtained from Eqn. 4.22 . If electrons can travel radially by the amount given in Eqn. 4.23 then they can contribute significantly to the photoemitted current. Because these electrons have spent a longer time inside the semiconductor, they are likely to have suffered spin relaxation. Thus the contribution of these electrons to the photoemission current is expected to lower the beam polarization. When a photocathode is fully illuminated into $\mathrm{CL}$ there can be no contribution of low polarization electrons to the photoemission which is in agreement with the laboratory observations. 


\section{Chapter 5}

\section{Conclusion}

The GTL with its new Mott polarimeter has become a useful tool for the study and development of GaAs photocathodes. Both the charge production and the polarization characteristics of new photocathodes can be tested before they are used in the accelerator. Moreover, the GTL Mott polarimeter was calibrated with a standard source of polarized electrons that was measured at various institutions. Thus SLAC now has the capability of knowing what a polarized source tested or developed at a different institution would measure in the GTL. The tests performed for this thesis have shown which spin relaxation models are most relevant to the polarization of thin photocathodes. In addition, the $\mathrm{CL}$ tests seem to confirm the ideas of Spicer and Herrera about the accumulation of charge at the surface.[26] Most surprising, however, was the result that changes in the photoemission properties due to charge saturation can also affect the polarization characteristics of GaAs photocathodes. The last section of this chapter will discuss some future experiments that can improve the charge production and polarization of GaAs photocathodes.

The tests demonstrating the way in which the polarization depends on the photocathode temperature showed that low temperatures do not greatly reduce the effect of the spin relaxation mechanisms. At first this result was surprising since the BAP and DP mechanisms have a strong temperature dependence. However, both of these mechanisms require some time to reduce the polarization of electrons in the conduction band. Photoexcited electrons in thin photocathodes do not reside in the conduction band long enough to be affected by these mechanisms.

Changes to the vacuum level of a photocathode affect not only the $\mathrm{QE}$ but also the polarization of emitted electrons. One possible explanation for this behavior is that the vacuum level works as a filter of electrons that have lost both energy and polarization in the 
band-bending region. High vacuum levels (i.e. low $Q E$ ) produced the highest polarization in strained and unstrained photocathodes. Unfortunately, low vacuum levels (i.e. high $\mathrm{QE}$ ) are desired to produce high intensity beams.

Several new measurements on the charge production of these photocathodes showed that saturation indeed occurs when charge is accumulated on the surface. This surface charge takes some time to recombine to the valence band. During this period of time the photocathode is unable to produce high intensity beams.

The most surprising experimental result was the low polarization that occurs when the photocathode is illuminated with a high intensity laser pulse. It seems that once the photocathode is driven into saturation, electrons photoexcited into the conduction band can still escape if they diffuse to a non-saturated region. Since these electrons have spent a longer period of time inside the semiconductor it is likely that they have suffered spin relaxation.

The results obtained in this dissertation can help to map the strategy for the future development of high polarization and high charge-producing photocathodes. If temperature does not seem to affect much the polarization of thin photocathodes, then what is causing their depolarization? Some authors have suggested that the small depolarization (up to $10 \%$ relative) that these photocathodes suffer can be attributed to spin interactions in the band-bending region.[5][45] For this reason it is believed that utilizing an arsenic terminated (111B) crystal orientation, which has the smallest band-bending region, will help to reduce spin relaxation in GaAs. Figure 5.1 shows the band-bending regions for the various surface orientations. In the (111B) crystal electrons approaching the surface will have the least interaction with the upper conduction band levels. Thus electrons will be less likely to be reflected at the surface and more likely to be transmitted. Electrons with a minimum amount of interaction at the surface will spend less time inside the semiconductor and thus will be less likely to suffer spin relaxation. Experimental evidence supports the idea that the (111B) surface in GaAs yields the highest polarization.[43] Moreover, Fig. 5.2 shows how experimental evidence also supports the idea that the (111B) surface yields the highest $\mathrm{QE}$. The advantages of (111B) GaAs photocathodes can be summarized as follows:

- A higher escape probability due to reduced scattering at the surface/band-bending region improves the $\mathrm{QE}$. 

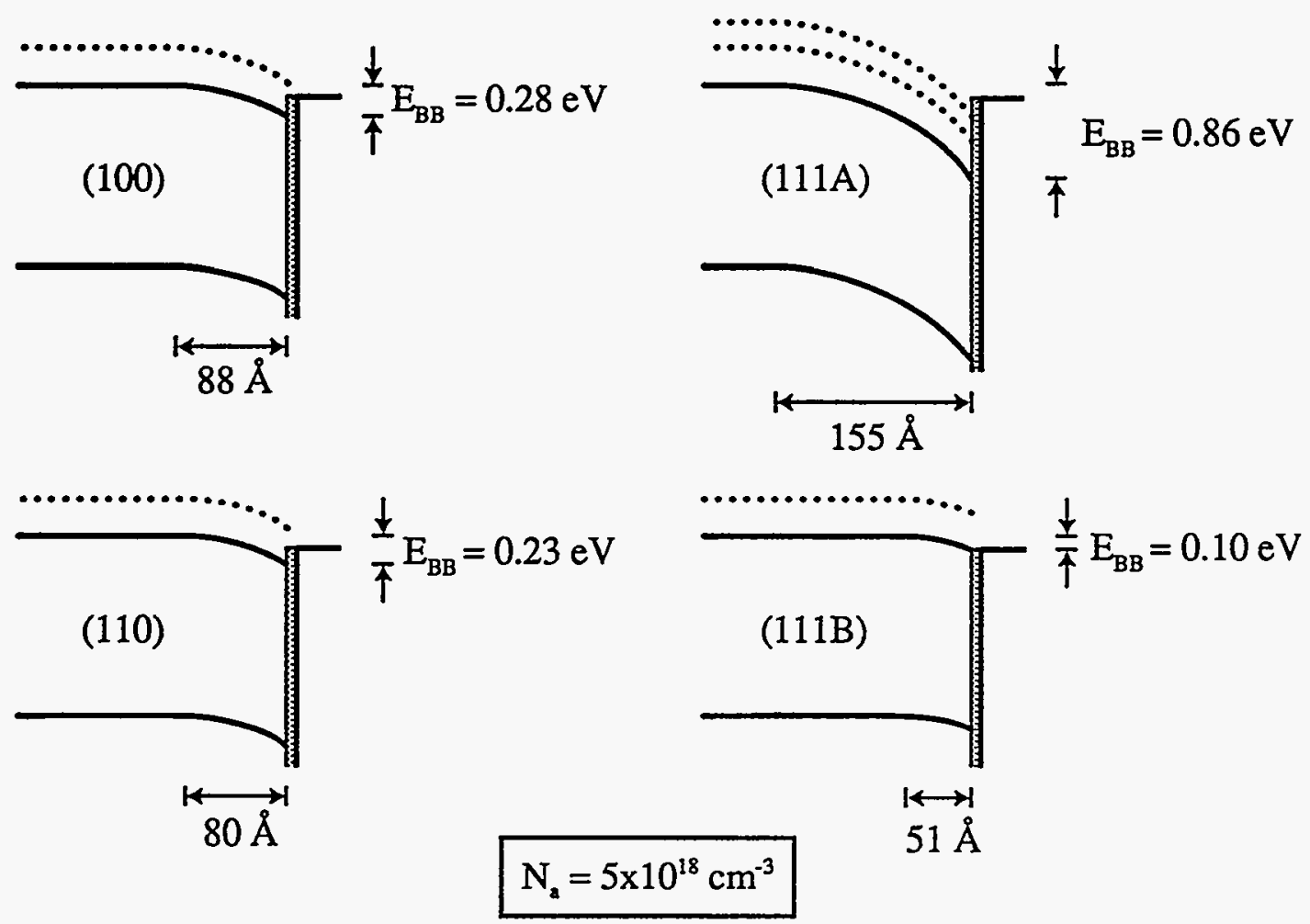

Figure 5.1: Band-bending region for various GaAs surface orientations.[10]

- Electrons spending a shorter amount of time in the band-bending region will suffer less spin relaxation.

Thus it would be very desirable to develop strained (111B) GaAs photocathodes because they have the potential for higher polarization and higher charge production. The main points of concern are whether this type of material can be grown and whether it can be activated easily.

Another technique which improves the $\mathrm{QE}$ and charge production of a photocathode is to grow a thin metallic layer on its surface.[26][49] The metallic layer will add continuous surface states. Thus electrons getting trapped at the surface will be able to recombine quickly to the valence band. Fewer electrons trapped at the surface will reduce charge saturation.

A number of interesting experiments that can further the understanding of $\mathrm{GaAs}$ photocathodes can now be performed in the GTL. Some of these include: 


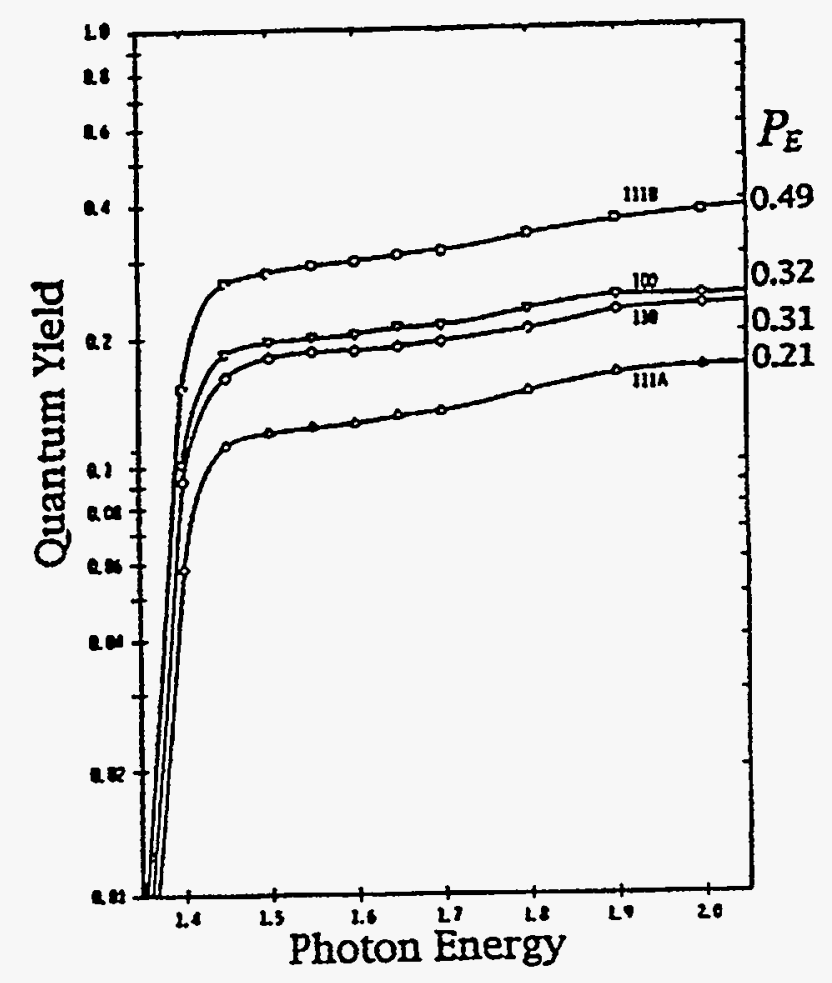

Figure 5.2: $Q E$ dependence on surface orientation for GaAs photocathodes.[10]

- The study of $\mathrm{QE}, \mathrm{CL}$, and polarization versus surface orientation for thin GaAs photocathodes. In these experiments it is of outmost importance to preserve the surface orientation of the photocathode. Thus the activation temperature should never exceed the surface reconstruction threshold.

- The study of $\mathrm{QE}, \mathrm{CL}$, and polarization of strained (111B) GaAs photocathodes.

- The study of how the doping concentration affects the $\mathrm{QE}$ and strain of thin GaAs photocathodes.

- The study of polarization versus laser intensity for areas of high and low QE. Repeat the study of polarization across the electron bunch.

- The study of $\mathrm{QE}, \mathrm{CL}$, and polarization of palladium- or silver-coated GaAs photocathodes.

These experiments can be enhanced by utilizing a tunable-wavelength laser. Such a tunable light source can make it easier to measure changes in the $\mathrm{QE}$ and polarization as a function of the work function of the semiconductor. It is hoped that the experiments 
mentioned above will help to develop photocathodes with improved polarization and charge production capabilities. 


\section{Appendix A}

\section{Electromagnetic Transitions in GaAs}

The strength of photon-induced electron transitions between the valence and conduction bands in GaAs can be computed using the dipole approximation.[1][50] The photon energy must be equal to or greater than the energy difference between the bands. For the production of longitudinaly polarized electrons, the photon polarization has to be circular (angular momentum $= \pm \hbar$ ). Figure A.1 illustrates the energy diagram of the electromagnetic transitions between the valence and conduction bands in GaAs. The eigenfunctions chosen to represent the electrons in the bands are those that diagonalize the spin-orbit Hamiltonian and given by the representation $\left|j, m_{j}\right\rangle$, the angular distribution described by spherical harmonics. The eigenfunctions of the valence and conduction bands are given by

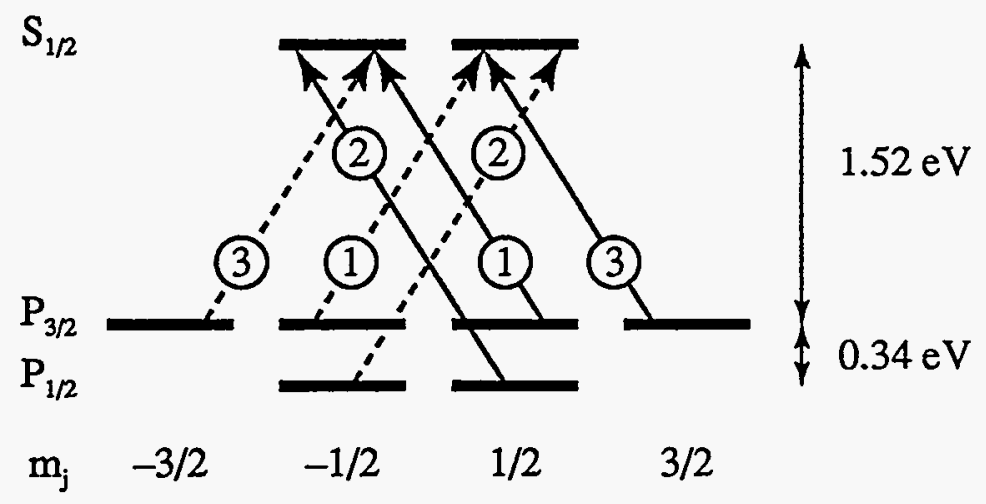

Figure A.1: Electromagnetic transitions at the $\Gamma$ point of GaAs for $T=0{ }^{\circ} \mathrm{K}$. The numbers inside the circles indicate the relative strength of the transitions. The solid and dashed arrows represent photons with angular momentum $-\hbar$ and $+\hbar$, respectively. 


$$
\begin{aligned}
\left|\frac{3}{2}, \frac{3}{2}\right\rangle & =Y_{1}^{1} \uparrow & \left|\frac{3}{2},-\frac{3}{2}\right\rangle & =Y_{1}^{-1} \downarrow \\
\left|\frac{3}{2}, \frac{1}{2}\right\rangle & =\sqrt{\frac{1}{3}} Y_{1}^{1} \downarrow+\sqrt{\frac{2}{3}} Y_{1}^{0} \uparrow & \left|\frac{3}{2},-\frac{1}{2}\right\rangle & =\sqrt{\frac{2}{3}} Y_{1}^{0} \downarrow+\sqrt{\frac{1}{3}} Y_{1}^{-1} \uparrow \\
\text { C.B.: }\left\langle\frac{1}{2}, \frac{1}{2}, \frac{1}{2}\right\rangle & =-\sqrt{\frac{1}{3}} Y_{1}^{0} \uparrow+\sqrt{\frac{2}{3}} Y_{1}^{1} \downarrow & \left|\frac{1}{2},-\frac{1}{2}\right\rangle & =\sqrt{\frac{1}{3}} Y_{1}^{0} \downarrow-\sqrt{\frac{2}{3}} Y_{1}^{-1} \uparrow
\end{aligned}
$$

where the $Y_{l}^{m}$ are the spherical harmonics and the arrows represent the spin orientation with respect to the $\mathrm{z}$ axis. The electric field of circularly polarized light is given by

$$
E_{0}(\hat{\mathbf{x}} \pm i \hat{\mathbf{y}}) e^{i(\mathbf{k} \cdot \mathbf{r}-\omega t)}
$$

where $E_{0}$ is the magnitude of the electric field, $\mathbf{k}$ is the wave vector perpendicular to the electric field, and $\omega$ is the angular frequency.[16] For unstrained GaAs, the relevant transition at the $\Gamma$ point that produces non-zero polarization is $P_{3 / 2} \rightarrow S_{1 / 2}$. For negative circularly polarized light, the matrix elements of the electromagnetic transition in the dipole approximation are proportional to [50]

$$
\begin{gathered}
M_{\frac{3}{2} \rightarrow \frac{1}{2}} \propto\left\langle\frac{1}{2}, \frac{1}{2}|(\hat{\mathbf{x}}-i \hat{\mathbf{y}}) \cdot \mathbf{r}| \frac{3}{2}, \frac{3}{2}\right\rangle \propto \int\left(Y_{0}^{0}\right)^{*} \uparrow e^{-i \phi} Y_{1}^{0} \uparrow d \Omega=-\sqrt{\frac{3}{2}} \\
M_{\frac{1}{2} \rightarrow-\frac{1}{2}} \propto\left\langle\frac{1}{2},-\frac{1}{2}|(\hat{\mathbf{x}}-i \hat{\mathbf{y}}) \cdot \mathbf{r}| \frac{3}{2}, \frac{1}{2}\right\rangle=\int\left(Y_{0}^{0}\right)^{*} \downarrow e^{-i \phi}\left(\sqrt{\frac{1}{3}} Y_{1}^{1} \downarrow+\sqrt{\frac{2}{3}} Y_{1}^{0} \uparrow\right) d \Omega=-\sqrt{\frac{1}{2}}
\end{gathered}
$$

The probability of transition is proportional to the square of the matrix elements. The ratio of the two transitions is then given by

$$
\left|\frac{M_{\frac{3}{2} \rightarrow \frac{1}{2}}}{M_{\frac{1}{2} \rightarrow-\frac{1}{2}}}\right|^{2}=\frac{3}{1}
$$


A similar ratio can be found for transitions with positive circularly polarized light yielding the ratio

$$
\left|\frac{M_{-\frac{3}{2} \rightarrow-\frac{1}{2}}}{M_{-\frac{1}{2} \rightarrow \frac{1}{2}}}\right|^{2}=\frac{3}{1}
$$

A summary of the various strengths for the $P_{3 / 2} \rightarrow S_{1 / 2}$ transition is shown in Fig. A.1 


\section{Appendix B}

\section{Stress Perturbation of the GaAs Crystal Lattice}

The symmetry of the orbital angular momentum $L=1$ creates a three-fold degenerate energy state. However, when the spin of the electron is taken into account, there are states where the orbital angular momentum and the spin angular momentum add up $(J=1$ $+1 / 2=3 / 2)$ or substract $(J=1-1 / 2=1 / 2)$. The energy associated with this arrangement is known as the spin-orbit interaction and is given by

$$
H_{\text {s.o. }}=\frac{1}{2 m^{2} c^{2}} \frac{1}{r} \frac{d V}{d r} \mathbf{L} \cdot \mathbf{S}
$$

where $m$ is the mass of the electron, $c$ is the speed of light, $r$ is the separation between the electron and the nucleus, $V$ is the potential distribution, $L$ is the orbital angular momentum operator, and $\mathbf{S}$ is the spin angular momentum operator.[40][1] The energy of the interaction described by Eqn. B.1 accounts for the separation of the $P_{3 / 2}$ and $P_{1 / 2}$ states. In a similar way, the introduction of a non-isotropic stress destroys the orbital symmetry in a cubic semiconductor lattice and breaks up the $P_{3 / 2}$ degeneracy. For a small perturbation, the energy in the stress interaction is conveniently expressed in terms of the components of the strain tensor $\varepsilon_{i j}$ [18] The orbital strain Hamiltonian for a band at $\mathbf{k}=0$ has been derived and is given by

$$
H_{\varepsilon}=-a\left(\varepsilon_{x x}+\varepsilon_{y y}+\varepsilon_{z z}\right)-3 b\left[\left(L_{x}^{2}-\frac{1}{3} L^{2}\right) \varepsilon_{x x}+c . p .\right]-\frac{6 d}{\sqrt{3}}\left[\left\{L_{x} L_{y}\right\} \varepsilon_{x y}+c . p .\right]
$$

where c.p. stands for cyclic permutation with respect to the indices $x, y$, and $z$ and where 


$$
\left\{L_{x}, L_{y}\right\}=\frac{1}{2}\left(L_{x} L_{y}+L_{y} L_{x}\right)
$$

The constants $a, b$, and $d$ stand for the hydrostatic pressure deformation potential and the uniaxial deformation potentials for strains of tetragaonal symmetry and rhombohedral symmetry, respectively. This section will be concerned with the deformation of the valence band and not with the deformation of the conduction band.

The creation of stress in our photocathodes is done through the epitaxial growth of GaAs on a smaller lattice compound such as $\mathrm{GaAs}_{\mathbf{x}} \mathrm{P}_{1-\mathrm{x}}$. This procedure was shown in Fig. 2.10 of section 2.3.1. The lattice mismatch between the two compounds will cause a biaxial stress on the GaAs lattice in the (100) and (010) directions. In practice, the lattice constant of the substrate is selected such that it creates a large and uniform strained layer. The strain components of the ideal stress are given by [51][18][19]

$$
\begin{aligned}
& \varepsilon_{z x}=\varepsilon_{y y}=-\varepsilon=\frac{a-a_{0}}{a} \\
& \varepsilon_{z z}=+\frac{2 C_{12}}{C_{11}} \varepsilon \\
& \varepsilon_{x y}=\varepsilon_{y z}=\varepsilon_{z x}=0
\end{aligned}
$$

where $a_{0}$ and $a$ are the lattice constants of the substrate $\left(\mathrm{GaAs}_{\mathrm{x}} \mathrm{P}_{1-\mathrm{x}}\right)$ and the strained layer (GaAs), respectively. The $\mathrm{C}_{\mathrm{ij}}$ are the elastic stiffness constants of the material. Substituting (B.3) into (B.2) results in the simplified Hamiltonian

$$
H_{\varepsilon}=2 a \varepsilon\left(\frac{C_{11}-C_{12}}{C_{11}}\right)-3 b \varepsilon\left(\frac{C_{11}+2 C_{12}}{C_{11}}\right)\left(L_{z}^{2}-\frac{1}{3} \mathrm{~L}^{2}\right)
$$

The first term in Eqn. B.4 causes a shift in the center of gravity while the second term causes the split of the degenerate valence band. The minimum Hamiltonian required to describe the valence band energy distribution is

$$
H=H_{\text {s.o. }}+H_{\varepsilon}
$$


The matrix elements which describe the Hamiltonian in Eqn. B.5 are given by

$$
\left[\begin{array}{ccc}
\frac{1}{3} \Delta+\alpha+\beta & 0 & 0 \\
0 & \frac{1}{3} \Delta+\alpha-\beta & \sqrt{2} \beta \\
0 & \sqrt{2} \beta & -\frac{2}{3} \Delta+\alpha
\end{array}\right]
$$

where

$$
\alpha=-2 a \varepsilon\left(\frac{C_{11}-C_{12}}{C_{11}}\right), \beta=b \varepsilon\left(\frac{C_{11}+2 C_{12}}{C_{11}}\right)
$$

and $\Delta$ is the spin-orbit energy split between the $P_{3 / 2}$ and $P_{1 / 2}$ states. The terms along the diagonal are the first-order energy shifts. The off-diagonal terms indicate that there are higher order energy shifts and some mixing of the base states. Note that the eigenvalue $\left|\frac{3}{2}, \frac{3}{2}\right\rangle$ is not coupled by strain to the other two states. The diagonalization of the Hamiltonian in Eqn. B.6 yields the following energy eigenvalues (up to second order in strain)

$$
\begin{aligned}
& E^{(1)}=\frac{1}{3} \Delta+\alpha+\beta \\
& E^{(2)}=\frac{1}{3} \Delta+\alpha-\beta+2 \frac{\beta^{2}}{\Delta} \\
& E^{(3)}=-\frac{2}{3} \Delta+\alpha-2 \frac{\beta^{2}}{\Delta}
\end{aligned}
$$

The corresponding (unnormalized) eigenfunctions are given to first order in strain as 


$$
\begin{aligned}
& \psi_{1}=\left|\frac{3}{2}, \frac{3}{2}\right\rangle \\
& \psi_{2}=\left|\frac{3}{2}, \frac{1}{2}\right\rangle+\frac{\sqrt{2} \beta}{\Delta}\left|\frac{1}{2}, \frac{1}{2}\right\rangle \\
& \psi_{3}=\left|\frac{3}{2}, \frac{1}{2}\right\rangle-\frac{\sqrt{2} \beta}{\Delta}\left|\frac{1}{2}, \frac{1}{2}\right\rangle
\end{aligned}
$$

The energy difference between the first and second energy eigenvalues represent energy split of the $P_{3 / 2}$ state and is given by

$$
\Delta_{P_{3 / 2}}=2 \beta
$$

where second order terms in strain have been ignored for strain values on the order of $1 \%$.

\section{Shear Strain}

It has been found that the strained GaAs photocathodes can develop a shear strain during their growth.[52] This shear strain will change the photoemissive properties of the semiconductor. A GaAs lattice structure with shear strain is shown in Fig. B.1. The Hamiltonian perturbation term due to the shear strain is given by

$$
H_{\text {shear }}=-\frac{6 d}{\sqrt{3}}\left\{L_{x} L_{y}\right\} \varepsilon_{x y}
$$

The eigenvector that yields non-zero matrix elements is given by

$$
\Psi=\left(\begin{array}{l}
\left|\frac{3}{2}, \pm \frac{3}{2}\right\rangle \\
\left|\frac{3}{2}, \mp \frac{1}{2}\right\rangle \\
\left|\frac{1}{2}, \mp \frac{1}{2}\right\rangle
\end{array}\right\rangle
$$



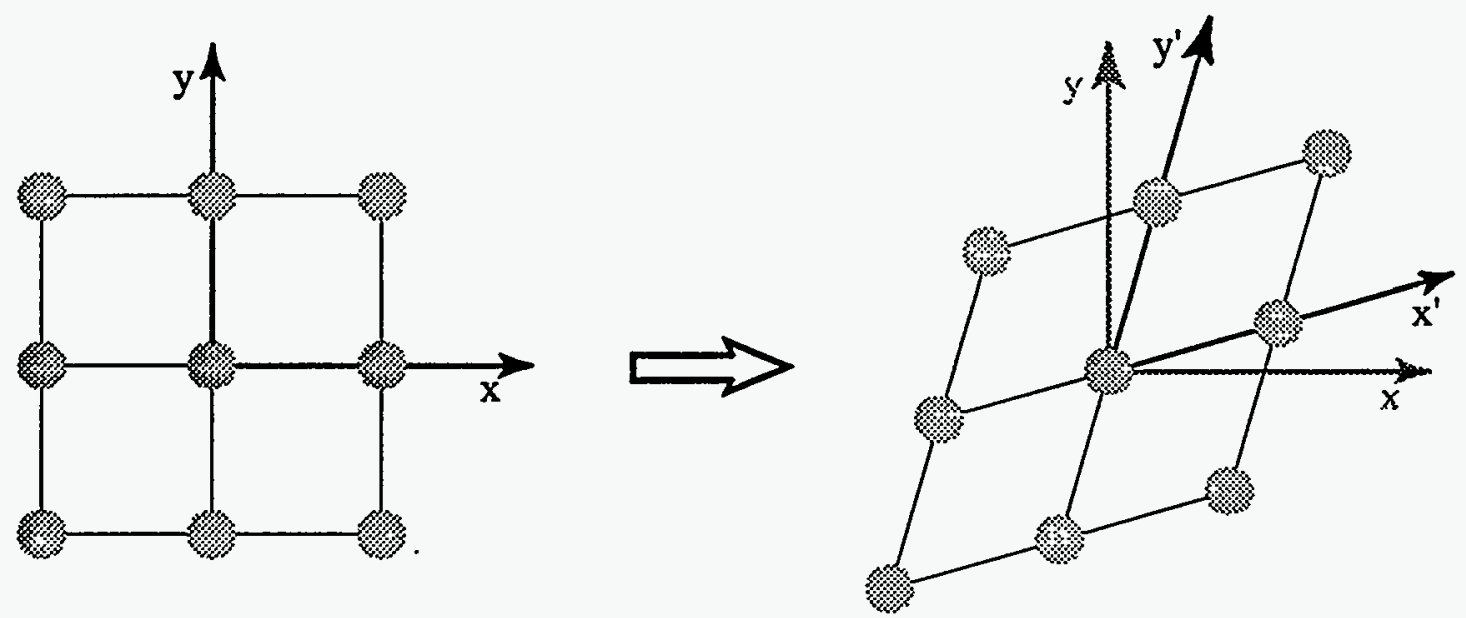

Figure B.1: Formation of shear stain in the GaAs crystal lattice.

The corresponding matrix elements of the shear Hamiltonian in Eqn. B.10 are given by

$$
\left(\begin{array}{ccc}
0 & \mp \mathrm{i} \delta & +\mathrm{i} \sqrt{2} \delta \\
\pm \mathrm{i} \delta & 0 & 0 \\
-\mathrm{i} \sqrt{2} \delta & 0 & 0
\end{array}\right)
$$

where $\delta=d \varepsilon_{x y}$ is the term arising from the shear strain. The total Hamiltonian equation for the strained samples becomes $H=H_{\text {s.o. }}+H_{\varepsilon}+H_{\text {shear }}$ and the matrix elements are given by

$$
\left(\begin{array}{ccc}
\frac{1}{3} \Delta+\alpha+\beta & \mp \mathrm{i} \delta & +\mathrm{i} \sqrt{2} \delta \\
\pm \mathrm{i} \delta & \frac{1}{3} \Delta+\alpha-\beta & 0 \\
-\mathrm{i} \sqrt{2} \delta & 0 & -\frac{2}{3} \Delta+\alpha
\end{array}\right)
$$

The section of the Hamiltonian which mixes the heavy and light hole states is just 


$$
\left(\begin{array}{cc}
\frac{1}{3} \Delta+\alpha+\beta & \mp \mathrm{i} \delta \\
\pm \mathrm{i} \delta & \frac{1}{3} \Delta+\alpha-\beta
\end{array}\right)
$$

The eigenfunctions that diagonalize the above Hamiltonian are

$$
\begin{aligned}
& \psi_{1}=\left|\frac{3}{2}, \frac{3}{2}\right\rangle-\mathrm{i} \frac{\delta}{\beta}\left|\frac{3}{2},-\frac{1}{2}\right\rangle \\
& \psi_{2}=\left|\frac{3}{2},-\frac{1}{2}\right\rangle-\mathrm{i} \frac{\delta}{\beta}\left|\frac{3}{2}, \frac{3}{2}\right\rangle
\end{aligned}
$$

and their corresponding energy levels are given by

$$
\begin{aligned}
& E_{1}=\frac{1}{3} \Delta+\alpha+\sqrt{\beta^{2}+\delta^{2}} \\
& E_{2}=\frac{1}{3} \Delta+\alpha-\sqrt{\beta^{2}+\delta^{2}}
\end{aligned}
$$

If the photocathode is illuminated with light of energy equal to the difference between the conduction band and the highest valence band level, pure circularly polarized light will not produce any new transitions. In other words,

$$
\begin{gathered}
M_{\psi_{1} \rightarrow s_{42}}^{-} \propto\left\langle\frac{1}{2}, \frac{1}{2}\left|(\hat{\mathbf{x}}-i \hat{\mathbf{y}}) \cdot \mathbf{r}\left(\left|\frac{3}{2}, \frac{3}{2}\right\rangle-i \frac{\delta}{\beta}\left|\frac{3}{2},-\frac{1}{2}\right\rangle\right)\right.\right. \\
=\left\langle\frac{1}{2}, \frac{1}{2}|(\hat{\mathbf{x}}-i \hat{\mathbf{y}}) \cdot \mathbf{r}| \frac{3}{2}, \frac{3}{2}\right\rangle=M_{P_{32} \rightarrow s_{42}}^{-}
\end{gathered}
$$

Thus the shear strain does not affect electromagnetic transitions of purely right or purely left circular polarization. Electromagnetic transitions with linearly polarized ligth are a different matter. Since linearly polarized light can be thought of as a combination of right 
and left circular polarization, the transitions between the valence band and the conduction band are expected to be dependent on the orientation of the linear polarization. The electric field vector for linear polarization with an angle $\phi_{0}$ with respect to the $\mathrm{x}$ axis is given by

$$
\mathbf{E} \propto\left(e^{-i \phi_{0}} \hat{\varepsilon}_{+}+e^{i \phi_{0}} \hat{\varepsilon}_{-}\right) e^{i(\mathbf{k} \cdot \mathbf{r}-\omega x)}
$$

where $\hat{\varepsilon}_{ \pm}=\hat{\mathbf{x}} \pm i \hat{\mathbf{y}}$ are the nominal vectors for right and left circular polarization.[16] If the transition matrix for linear polarization illumination is given by

$$
M_{\psi_{1} \rightarrow S_{12}}^{\text {linear }} \propto\left\langle\frac{1}{2}, \frac{1}{2}\left|\left(e^{-i \phi_{0}} \hat{\varepsilon}_{+}+e^{i \phi_{0}} \hat{\varepsilon}_{-}\right) \cdot \mathrm{r}\left(\left|\frac{3}{2}, \frac{3}{2}\right\rangle-i \frac{\delta}{\beta}\left|\frac{3}{2},-\frac{1}{2}\right\rangle\right)\right.\right.
$$

then the transition probability yields

$$
\left|M_{\psi_{1} \rightarrow \frac{1}{2}}^{\text {linear }}\right|^{2} \propto \frac{\pi^{2}}{32}\left\{3+\left(\frac{\delta}{\beta}\right)^{2}+2 \sqrt{3} \frac{\delta}{\beta} \sin 2 \phi_{0}\right\}
$$

This angular dependence of the transition probability will affect the $\mathrm{QE}$; as the angle of linear polarization $\phi_{0}$ is changed, the Q.E. will change with a $\sin 2 \phi_{0}$ dependence. Robin Mair, Takashi Maruyama, Greg Mulholland, and Huan Tang reported experimental observations of relative changes in $\mathrm{QE}$ with linear polarization of $\pm 14 \%$. These changes in QE indicate that the relationship between $\beta$ and $\delta$ (utilizing Eqn. B.20 and assuming $\beta$ $>\delta$ ) is on the order of

$$
\frac{\delta}{\beta} \approx 0.12
$$

Some of the other references used in the production of this section include [53][54][55][56][57][58]. 


\section{Appendix C}

\section{Motion of the Electron Spin}

The first correct derivation of the electron spin motion under external forces was done by Thomas.[59] In particular, he pointed out that a rotation of the coordinate system induces a rotation of the underlying vector in the laboratory frame.[60] In the case of the spin, this motion is known as the Thomas precesion and it helps to explain qualitatively the spin-orbit interaction in atomic nuclei.[16] This appendix will include an outline of the derivation of the rate of rotation of the electron spin and it will discuss the relationship between the spin rotation and the spin-orbit interaction.

\section{Equation of Motion of the Spin Vector}

The spin $\mathbf{S}$ is typically defined in the rest frame of the particle while the space, the time, and the electromagnetic fields are defined in the laboratory or inertial frame. ${ }^{\dagger}$ Most interactions involving the spin are usually evaluated in its rest frame where equations are simpler. Once a particle distribution is found, the result is converted to the laboratory frame using Lorentz transformations.

The total time rate of change of the spin, or more generally, of any vector as seen by an observer in the laboratory frame is equal to the rate in the rest frame plus the effect of rotation of the body axes:

$$
\left(\frac{d \mathbf{S}}{d t}\right)_{l a b}=\left(\frac{d \mathbf{S}}{d t}\right)_{\text {restrame }}+\omega_{T} \times \mathbf{S}
$$

† With the exception of the spin S, all quantities in the rest frame will be identified by the prime symbol:' 
where $\omega_{T}$ is the angular velocity of rotation of the spin axes derived by Thomas. The equation of motion of the spin in the rest frame is given by

$$
\frac{d \mathbf{S}}{d t^{\prime}}=\mu \times \mathbf{B}^{\prime}
$$

where $\mathbf{B}^{\prime}$ is the magnetic field in that frame and $\mu$ is the magnetic moment of the electron given by

$$
\mu=\frac{g e}{2 m} \mathbf{S}
$$

where $g$ is the gyromagnetic factor with the value $g=2.00232, e$ is the charge, and $m$ is the mass. By identifying $\delta t=\gamma \delta t^{\prime}$, the rate of rotation of the spin in its rest frame is given by

$$
\left(\frac{d \mathbf{S}}{d t}\right)_{\text {restrame }}=\frac{g}{2} \frac{e}{y m} \mathbf{S} \times \mathbf{B}^{\prime}
$$

where $\gamma$ is the Lorentz energy factor. Substitution of Eqn. C. 4 into C. 1 yields

$$
\frac{d \mathbf{S}}{d t}=\Omega_{0} \times \mathbf{S}
$$

where $\Omega_{0}$ is the net rate of spin precesion given by

$$
\Omega_{0}=\omega_{T}-\frac{g}{2} \frac{e}{\gamma m} \mathbf{B}^{\prime}
$$

The magnetic field in the rest frame can be obtained from Lorentz transformations and is given by [16] 


$$
\mathbf{B}^{\prime}=\gamma\left(\mathbf{B}-\frac{\mathbf{v} \times \mathbf{E}}{c^{2}}\right)+(1-\gamma) \frac{\mathbf{v}(\mathbf{v} \cdot \mathbf{B})}{v^{2}}
$$

where $\mathbf{E}$ and $\mathbf{B}$ are the electromagnetic fields in the laboratory frame.[16]

The precesion of the rest frame axes with respect to the laboratory makes the spin vector have a total rate of change with respect to the laboratory axes given by

$$
\omega_{T}=(\gamma-1) \frac{\mathbf{a} \times \mathbf{v}}{v^{2}}
$$

where $\mathbf{a}$ is the acceleration of the spin vector in the laboratory frame.[16] This precession rate is purely kinematical. If an acceleration exists that is transverse to the velocity $\mathbf{v}$, there will be a precession regardless of other effects such as precession of the magnetic moment in a magnetic field.

The acceleration of a charged particle can be obtained from its equation of motion[61]

$$
\frac{d \mathbf{p}}{d t}=e(\mathrm{E}+\mathrm{v} \times \mathbf{B})
$$

The particle momentum can be expressed in terms of the velocity and kinetic energy as

$$
\mathbf{p}=y m \mathbf{v}=\frac{\varepsilon}{c^{2}} \mathbf{v}
$$

The rate of change of the kinetic energy is given by

$$
\frac{d \varepsilon}{d t}=\mathbf{v} \cdot \frac{d \mathbf{p}}{d t}=e \mathbf{v} \cdot \mathbf{E}
$$

where only the electric field does work on the charge. Since the force exerted by the magnetic field is always perpendicular to the velocity, it does no work on the charge. 
Substitution of Eqns. C.10 and C.11 into C.9 produces the acceleration in the laboratory frame

$$
\mathbf{a}=\frac{d \mathbf{v}}{d t}=\frac{e}{y_{m}}\left[\mathbf{E}+\mathbf{v} \times \mathbf{B}-\frac{1}{c^{2}} \mathbf{v}(\mathbf{v} \cdot \mathbf{E})\right]
$$

The rate of spin precession is obtained by substituting Eqn. C.7, C.8, and C.12 into C.6:

$$
\Omega_{0}=-\frac{e}{m m}\left\{\left[1+\gamma\left(\frac{g-2}{2}\right)\right] \mathbf{B}-(\gamma-1)\left(\frac{g-2}{2}\right) \frac{\mathbf{v}(\mathbf{v} \cdot \mathbf{B})}{v^{2}}+\gamma\left(\frac{\gamma}{\gamma+1}-\frac{g}{2}\right) \frac{\mathbf{v} \times \mathbf{E}}{c^{2}}\right\}
$$

One important point to realize is how the longitudinal polarization changes in an electromagnetic field. The rate of change of the longitudinal polarization is given by

$$
\frac{d}{d t}(\hat{\mathbf{v}} \cdot \mathbf{S})=\hat{\mathbf{v}} \cdot \frac{d \mathbf{S}}{d t}+\frac{1}{v}[\mathbf{S}-(\hat{\mathbf{v}} \cdot \mathbf{S}) \hat{\mathbf{v}}] \cdot \frac{d \mathbf{v}}{d t}=\hat{\mathbf{v}} \cdot \frac{d \mathbf{S}}{d t}+\frac{1}{v} \mathbf{S}_{\perp} \cdot \frac{d \mathbf{v}}{d t}
$$

Substitution of Eqns. C.5, C.12, and C.13 into C.14 produces

$$
\frac{d}{d t}(\hat{\mathbf{v}} \cdot \mathbf{S})=-\frac{e}{m} \mathbf{S}_{\perp} \cdot\left[\left(\frac{g-2}{2}\right) \hat{\mathbf{v}} \times \mathbf{B}+\left(\frac{g}{2} \frac{v}{c^{2}}-\frac{1}{v}\right) \mathbf{E}\right]
$$

Note that for a particle with $g=2$ in a purely magnetic field, the spin precesses in such a way that the longitudinal polarization remains constant.

\section{Charged Particle in a Transverse Electromagnetic Field}

If there is no component of the electric field $E$ pararell to the velocity $\mathbf{v}$, then the acceleration in Eqn. C.12 becomes

$$
\frac{d \mathbf{v}}{d t}=\frac{e}{m}(\mathrm{E}+\mathbf{v} \times \mathbf{B})
$$


where the purely transverse acceleration does no work on the charge, hence there is no change in its energy. Equation C.16 can be written as a rotation of the velocity vector

$$
\frac{d \mathrm{v}}{d t}=\Omega_{\mathrm{c}} \times \mathrm{v}
$$

where the relativistic cyclotron frequency $\Omega_{\mathrm{C}}$ is given by

$$
\Omega_{\mathrm{c}}=-\frac{e}{m m}\left(\mathbf{B}-\frac{\gamma^{2}}{\gamma^{2}-1} \frac{\mathrm{v} \times \mathrm{E}}{c^{2}}\right)
$$

\section{Angle of Spin Precession}

In interactions dealing with spin, such as in Mott scattering, it is more convenient to express the spin orientation with respect to the velocity of the particle. Thus the precession of the spin with respect to the velocity is given by substracting the rate of precession of the velocity from the rate of precession of the spin: $\Omega=\Omega_{0}-\Omega_{\mathrm{c}}$

$$
\Omega=-\frac{e}{m}\left\{\gamma\left(\frac{g-2}{2}\right) \mathbf{B}-(\gamma-1)\left(\frac{g-2}{2}\right) \frac{\mathbf{v}(\mathbf{v} \cdot \mathbf{B})}{v^{2}}-\gamma\left[\left(\frac{g-2}{2}\right)-\frac{1}{\gamma^{2}-1}\right] \frac{\mathbf{v} \times \mathbf{E}}{c^{2}}\right\}
$$

In the case of the electrostatic spin rotator there is no magnetic field and the electric field is transverse to the velocity. Thus Eqns. C.18 and C.19 simplify to

$$
\begin{gathered}
\Omega_{c}=-\frac{e}{m} \frac{\gamma}{\gamma^{2}-1} \frac{\nu E}{c^{2}} \\
\Omega=-\frac{e}{m}\left[\left(\frac{g-2}{2}\right)-\frac{1}{\gamma^{2}-1}\right] \frac{\nu E}{c^{2}}
\end{gathered}
$$

The spin precession expressed in terms of the cyclotron frequency becomes 


$$
\Omega=\left[\left(\frac{g-2}{2}\right)-\frac{1}{\gamma^{2}-1}\right] \frac{\gamma^{2}-1}{\gamma} \Omega_{c}
$$

The angle of spin precession is obtained by multiplying Eqn. C.22 by the time to traverse the rotator:

$$
\Theta_{\text {prec }}=\left[\left(\frac{g-2}{2}\right)-\frac{1}{\gamma^{2}-1}\right] \frac{\gamma^{2}-1}{\gamma} \Theta_{\text {bend }}
$$

\section{Relation Between Thomas Precession and the Spin-Orbit Interaction}

The interaction energy of the electron spin in its rest frame is (to first order in $v$ )

$$
U^{\prime}=-\mu \cdot\left(\mathrm{B}-\frac{\mathbf{v} \times \mathbf{E}}{c^{2}}\right)
$$

The electric field force $e \mathbf{E}$ of an atom approximated as the negative gradient of a spherically symmetric average potential energy $V(r)$ is given by

$$
e \mathbf{E}=-\frac{\mathbf{r}}{r} \frac{d V}{d r}
$$

Thus a spin-interaction energy is obtained by substituting Eqn. C.25 into C.24:

$$
U^{\prime}=-\frac{g e}{2 m} \mathbf{S} \cdot \mathbf{B}+\frac{g}{2 m^{2} c^{2}}(\mathbf{S} \cdot \mathbf{L}) \frac{1}{r} \frac{d V}{d r}
$$

where $\mathbf{L}=\mathbf{r} \times \mathbf{p}$ is the orbital angular momentum of the electron. The spin-orbit term in Eqn. C.26 is twice too large, it is missing the contribution of the Thomas precession. The acceleration experienced by the electrons near a nucleus is due to the screened Coulomb field Eqn. C.25. The Thomas angular velocity Eqn. C.8 thus reduces to 


$$
\omega_{T} \approx-\frac{1}{2 m c^{2}} \mathbf{r} \times \mathbf{v} \frac{1}{r} \frac{d V}{d r}=-\frac{1}{2 m^{2} c^{2}} \mathbf{L} \frac{1}{r} \frac{d V}{d r}
$$

Note that the precession has the opposite sign of the orbital angular momentum. Since the spin energy terms have units of angular momentum times angular frequency, the new interaction energy is obtained by adding Eqn. C.27 to C.26 to obtain

$$
U^{\prime}=-\frac{g e}{2 m} \mathbf{S} \cdot \mathbf{B}+\frac{g-1}{2 m^{2} c^{2}}(\mathbf{S} \cdot \mathbf{L}) \frac{1}{r} \frac{d V}{d r}
$$

For $g=2$, the Thomas factor reduces the spin-orbit interaction by half. 


\section{Bibliography}

[1] J. Kessler, Polarized Electrons, Springer-Verlag, Berlin (1985).

[2] W. E. Spicer and A. Herrera-Gómez, "Modern Theory and Applications of Photocathodes," SLAC-PUB 6306 (1993), presented at the 1993 SPIE International Symposium on Imaging and Instrumentation, San Diego.

[3] M. Woods, et. al., "Observation of a Charge Limit for Semiconductor Photocathodes," J. Appl. Phys. 73, 8531 (1993).

[4] T. Maruyama, et. al., "Electron-Spin Polarization in Photoemission from Strained GaAs Grown on GaAs1-xPx," Phys. Rev. B46, 4261 (1992); H. Aoyagi, et. al., "Strain Dependence of Spin Polarization of Photoelectrons from a Thin GaAs Layer," Phys. Lett. A167, 415 (1992).

[5] F. Meier and B. P. Zakharchenya - Editors, Optical Orientation, G. E. Pikus and A.N. Titkov, Ch.3: Spin Relaxation under Optical Orientation in Semiconductors, North-Holland (1984).

[6] R. L. Bell and W. E. Spicer, "3-5 Compound Photocathodes: A New Family of Photoemitters with Greatly Improved Performance," Proc. of the IEEE 58, 1788 (1970).

[7] R. L. Bell, Negative Electron Affinity Devices, Clarendon Press, Oxford (1973).

[8] S. M. Sze, Physics of Semiconductor Devices, John Wiley \& Sons, New York (1981).

[9] J. S. Blakemore, et. al., "Semiconducting and other major properties of gallium arsenide," J. Appl. Phys. 53, R123 (1982).

[10] L. W. James, et. al., "Dependence on Crystalline Face of the Band Bending in $\mathrm{Cs}_{2} \mathrm{O}$-Activated GaAs," J. Appl. Phys. 42, 4976 (1971).

[11] K. W. Böer, Survey of Semiconductor Physics, Vol. I (Electrons and Other Particles in Bulk Semiconductors), Van Nostrand Reinhold, New York (1990).

[12] P. M. Morse and H. Feshbach, Methods of Theoretical Physics, Part I, McGrawHill, New York (1953). 
[13] E. H. Rhoderick and R. H. Williams, Metal-Semiconductor Contacts, Clarendon Press, Oxford (1988).

[14] V. I. Safarov and A. N. Titkov, "Optical Spin Orientation. Application to the Study of Photoelectron Kinetics," Physica 117B \& 118B, 497, (1983).

[15] E. M. Purcell, Electricity and Magnetism, Berkeley Physics Course Vol. 2, McGraw-Hill, New York (1985).

[16] J. D. Jackson, Classical Electrodynamics, John Wiley \& Sons, New York (1975).

[17] R. K. Willardson and A. C. Beer - Editors, Semiconductors and Semimetals, J. S. Escher, Ch. 3:NEA Semiconductor Photoemitters, Academic Press, New York (1981); L. Pauling, The Nature of the Chemical Bond, Cornell U. Press, New York (1967).

[18] A. Gavini and M. Cardona, "Modulated Piezoreflectance in Semiconductors," Phys. Rev. B1, 672 (1969).

[19] H. Asai and K. Oe, "Energy Band-gap Shift with Elastic Strain in $\mathrm{Ga}_{x} \mathrm{In}_{1 \cdot x} \mathrm{P}$ Epitaxial Layers on (001) GaAs Substrates," J. Appl. Phys. 54, 2052 (1983).

[20] H. Kato, et. al.., "Photoluminescence study of $\mathrm{In}_{\mathrm{x}} \mathrm{Al}_{1-\mathrm{x}} \mathrm{As}-\mathrm{GaAs}$ strained-layer superlattices," J. Appl. Phys. 59 (2), 588, 1986.

[21] J. Hornstra and W. J. Bartels, "Determination of the Lattice Constant of Epitaxial Layers of III-V Compounds," J. Crystal Growth 44, 513 (1978).

[22] G. Fishman and G. Lampel, "Spin Relaxation of Photoelectrons in p-type Gallium Arsenide," Phys. Rev. B16, 820 (1977).

[23] M. Erbudak and B. Reihl, "Depolarization of Photoelectrons Emitted from Optically Pumped GaAs," Appl. Phys. Lett., 33 (7), 584 (1978).

[24] S. Humphries, Charged Particle Beams, John Wiley \& Sons, New York (1990).

[25] P. Sáez, et al., "High Voltage Processing of the SLC Polarized Electron Gun," SLAC-PUB-6193 (1993), contributed to the 1993 Particle Accelerator Conference, Washington, D.C.

[26] A. Herrera-Gómez and W. E. Spicer, "Physics of High Intensity Nanosecond Electron Source," SLAC-PUB 6307 (1993), presented at the 1993 SPIE International Symposium on Imaging and Instrumentation, San Diego.

[27] R. Alley, et al., "The Stanford Linear Accelerator Polarized Electron Source," SLAC-PUB-95-6489 (1995), Nucl. Instr. and Meth. in Phys. Res. A 365 (1995). 
[28] R. Kirby, et al., "An In-situ Photocathode Loading System for the SLC Polarized Electron Gun," SLAC-PUB-6006 (1993), contributed to the 1993 IEEE Particle Accelerator Conference, Washington, D.C.

[29] K. R. Eppley, et al., Conf. Record of the 1991 IEEE Particle Accelerator Conf., San Francisco, CA.

[30] A. D. Yeremian, et al., 1992 Linear Accelerator Conf. Proc., Ottawa, Canada.

[31] J. Frisch, et al., "Operation of a Ti:Sapphire Laser for the SLAC Polarized Electron Source", SLAC-PUB-6165 (1993), contributed to the 1993 IEEE Particle Accelerator Conference, Washington, D.C.

[32] Most of the conceptual design and development of the thermionic and photocathode guns is attributed to Professor Roger Miller.

[33] D. Schultz, et al., "The Polarized Electron Source of the Stanford Linear Accelerator Center," SLAC-PUB-6606 (1992), presented at the 17th International Linear Accelerator Conference (LINAC94), Japan (1994).

[34] W. Herrmannsfeldt, "EGUN - An Electron Optics and Gun Design Program," SLAC Report 331 (1988).

[35] L. Klaisner, "Fast Faraday Cup," SLAC Memorandum, January 27, 1993.

[36] B. Montague, "Polarized Beams in High Energy Storage Rings," Physics Reports 113, 1 (1987).

[37] H. Wollnik, Optics of Charged Particles, Academic Press, Orlando (1987).

[38] B. M. Dunham and D. A. Engwall, NPL Polarized Source Group Technical Note \#91-3 (1991) (unpublished).

[39] B. M. Dunham, "Investigation of the Physical Properties of Photoemission Polarized Electron Sources for Accelerator Applications," Physics Ph. D. Thesis, U. of Illinois at Urbana-Champaign (1993).

[40] T. J. Gay and F. B. Dunning, "Mott Electron Polarimetry," Rev. Sci. Instrum. 63 (2), 1635 (1992).

[41] T. Gay, et al., "Extrapolation Procedures in Mott Electron Polarimetry," Rev. Sci. Instrum. 63 (1), 114 (1992).

[42] G. Mulholland, et al., "A Derivative Standard for Polarimeter Calibration," Proceedings of the IEEE Particle Accelerator Conference, Dallas, 1043 (1996). 
[43] S. Alvarado, et al., "Spin Polarized Photoemission from Molecular Beam Epitaxy-Grown Be-Doped GaAs," Z. Phys. B - Condensed Matter 44, 259 (1981).

[44] D. Pierce, et al., "GaAs spin polarized electron source," Rev. Sci. Instrum. 51 (4), 478 (1980).

[45] H. Riechert, et al., "Energy-Resolved Study of the Spin Precession in Photoemission from Activated (110) GaAs," Phys. Rev. B 38, 4136 (1988).

[46] M. G. Clark, "Interpretation of the Surface Boundary Conditions in the Diffusion Model for NEA Photoemission," J. Phys. D: Appl. Phys. 9, 2139 (1976).

[47] H. Tang, et al., "Experimental Studies of the Charge Limit Phenomenom in GaAs Photocathodes," Proceedings of the Workshop on Photocathodes for Polarized Electron Sources for Accelerators, Stanford, 345 (1993).

[48] M. Neuberger, Gallium Arsenide Data Sheets, DS-144, Electronic Properties Information Center, Hughes Aircraft Co., Culver City (1965).

[49] V. W. Aebi, et. al., "Photocathode Development for a 1300-nm Streak Tube," presented at the 1993 SPIE International Symposium on Imaging and Instrumentation, San Diego.

[50] H. A. Bethe and R. Jackiw, Intermediate Quantum Mechanics, Benjamin / Cummings, Menlo Park (1986).

[51] C. Kittel, Introduction to Solid State Physics, John Wiley \& Sons, New York (1986).

[52] R. Mair and H. Tang, "Strain and QE Measurements on Strained GaAs," SLAC Polarized Electron Source Seminar, November (1994).

[53] L. D. Landau and E. M. Lifshitz, The Classical Theory of Fields, Pergamon Press, Oxford (1975).

[54] R. L. Liboff, Introductory Quantum Mechanics, Holden-Day, Oakland (1980).

[55] S. Gasiorowicz, Quantum Physics, John Wiley \& Sons, Oakland (1974).

[56] W. J. Bartels and J. Hornstra, "Determination of the Lattice Constant of Epitaxial Layers of III-V Compounds," J. Crystal Growth 44, 513 (1978).

[57] F. H. Pollak and M. Cardona, "Piezo-Electroreflectance in Ge, GaAs, and Si," Phys. Rev. 172, 816 (1968). 
[58] P. Zorabedian, "Optical Pumping and Photoluminescence Detection of SpinPolarized Electrons in Uniaxially Stressed Gallium Arsenide," Ph.D. Dissertation, Stanford U., CA, SLAC-248 (1982).

[59] L. T. Thomas, Phil.Mag. 3, 1 (1927).

[60] H. Goldstein, Classical Mechanics, Addison-Wesley, Reading, MA (1980).

[61] L. D. Landau and E. M. Lifshitz, The Classical Theory of Fields, Pergamon Press, Oxford (1975). 